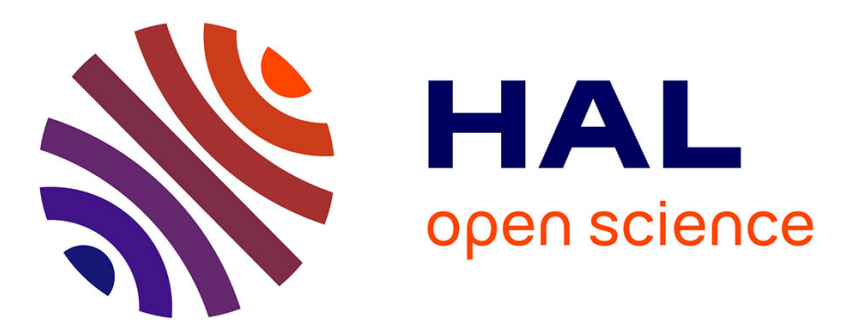

\title{
Determination of the surfaces radiosity temperatures in a square cavity with a square inner centred obstacle and a non-isothermal absorbing-emitting medium
}

\author{
V. Le Dez, M. Lazard, J.S. S Djeumegni
}

\section{- To cite this version:}

V. Le Dez, M. Lazard, J.S. S Djeumegni. Determination of the surfaces radiosity temperatures in a square cavity with a square inner centred obstacle and a non-isothermal absorbing-emitting medium. International Journal of Thermal Sciences, 2021, 165, pp.106953. 10.1016/j.ijthermalsci.2021.106953 . hal-03388981

\section{HAL Id: hal-03388981 \\ https://cnrs.hal.science/hal-03388981}

Submitted on 20 Oct 2021

HAL is a multi-disciplinary open access archive for the deposit and dissemination of scientific research documents, whether they are published or not. The documents may come from teaching and research institutions in France or abroad, or from public or private research centers.
L'archive ouverte pluridisciplinaire HAL, est destinée au dépôt et à la diffusion de documents scientifiques de niveau recherche, publiés ou non, émanant des établissements d'enseignement et de recherche français ou étrangers, des laboratoires publics ou privés. 


\title{
DETERMINATION OF THE SURFACES RADIOSITY TEMPERATURES IN A SQUARE CAVITY WITH A SQUARE INNER CENTRED OBSTACLE AND A NON-ISOTHERMAL ABSORBING-EMITTING MEDIUM
}

\author{
V. Le Dez ${ }^{1 *}$, M. Lazard ${ }^{1}$ and J.S. Djeumegni ${ }^{1}$ \\ ${ }^{1}$ Institut Pprime, Université de Poitiers, Centre National de la Recherche Scientifique, Ecole Nationale Supérieure \\ de Mécanique et Aérotechnique, 2 Rue Pierre Brousse, Bâtiment B25, TSA 41105, 86073 Poitiers Cedex 9, France \\ *Corresponding author. E-mail: vital.le.dez@univ-poitiers.fr
}

\begin{abstract}
An exact semi-analytical determination of the radiosity temperatures on the diffusely reflecting surfaces of a square cavity filled with a non-isothermal absorbing and emitting semi-transparent medium containing a centered reflecting opaque obstacle is proposed. One develops first the obstacle's surfaces radiosity temperatures formulation before detailing all the different possible configurations existing for the cavity's surfaces radiosity temperatures determination. Thanks to the radiosity technique, the integrals solutions of the radiative transfer equation are calculated for all listed cases. Then, the numerical results of the radiosity temperatures are obtained from a Gauss quadrature and an adequate meshing grid, following an iterative scheme. The effects of different parameters such as the size of the internal obstacle, the emissivities and the absorption coefficient on the cavity's radiosity temperatures behavior have also been investigated.
\end{abstract}

Keywords: Radiosity temperatures, Semi-analytical, Inner obstacle, Diffusely reflecting surfaces, Radiative transfer, Absorbing-emitting medium

\section{Nomenclature}

$\mathrm{Bis}_{n}, \mathrm{Cis}_{n} \quad$ Altaç angular integrated Bickley-Naylor functions

$\left(\boldsymbol{e}_{x}, \boldsymbol{e}_{y}, \boldsymbol{e}_{z}\right) \quad$ unit vectors of the $x, y, z$ directions

$H \quad$ length of the cavity (m)

$h \quad$ length of the obstacle $(\mathrm{m})$

(i) surface cells numbering

$q^{-} \quad$ incident radiative flux on a surface $\left(\mathrm{Wm}^{-2}\right)$

$I^{\leftarrow}(\Omega) \quad$ incoming intensity on a boundary surface $\left(\mathrm{Wm}^{-2} \mathrm{Sr}^{-1}\right)$

$I^{\rightarrow} \quad$ outgoing intensity leaving a boundary surface $\left(\mathrm{Wm}^{-2} \mathrm{Sr}^{-1}\right)$

$K i_{n} \quad$ Bickley-Naylor functions

$N_{t} \quad$ total number of cells on a cavity's surface

$M \quad$ number of cells on the length $\frac{H-h}{2}$

$P \quad$ number of cells on the length $\frac{h}{2}$

temperature $(\mathrm{K})$

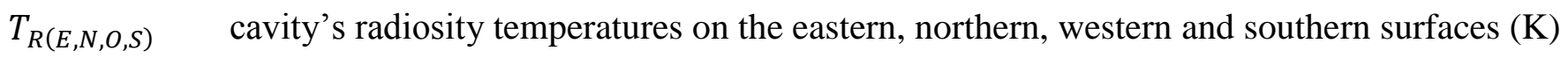

$T_{R \sigma(E, N, O, S)} \quad$ obstacle's radiosity temperatures on the four obstacle's surfaces $(\mathrm{K})$ 


\section{Greek letters and non Latin characters}

$\Delta x$

$\Delta y$

$\varepsilon$

$\rho$

$\kappa$

$\sigma$

$\varphi, \theta$

$\Omega$

$\Sigma_{O}, \Sigma_{N}, \Sigma_{E}, \Sigma_{S}$

$\sigma_{O}, \sigma_{N}, \sigma_{E}, \sigma_{S}$

$\tau_{0}, \tau_{0}^{\prime}, \tau^{\prime \prime}{ }_{0}$

$\xi_{E}^{+}, \xi_{E}^{-}, \xi_{N}^{+}, \xi_{S}^{+}$

$\alpha_{O}^{+}, \alpha_{\sigma_{E}}^{+}, \alpha_{O}^{-}, \alpha_{\sigma_{E}}^{-}, \alpha_{N}^{+}$

$\kappa$ characteristic cell length along the $x$ direction (m)

characteristic cell length along the $y$ direction (m), with $\Delta y=\Delta x$

surface emissivity

surface reflection factor

absorption coefficient $\left(\mathrm{m}^{-1}\right)$

Stephan-Boltzmann constant $\left(5.6710^{-8} \mathrm{Wm}^{-2} \mathrm{~K}^{-4}\right)$

angular description of the unit vector $\boldsymbol{\Omega}$

unit vector of radiation propagation

western, norther, eastern and southern boundary surfaces of the cavity

western, norther, eastern and southern boundary surfaces of the obstacle

the three characteristic optical depths

characteristic angles associated to the obstacle

some characteristic angles associated to the cavity

contribution of the semi-transparent medium to the radiosity temperatures

Subscripts (superscripts)

$E, N, O, S \quad$ east, north, west and south

\section{I - INTRODUCTION}

Closed or open cavities with diffusely reflecting boundary surfaces have been examined since it has been observed that the presence of radiation incoming from the walls yields to complex interactions with a convective fluid confined in the cavity [1], when the active boundaries are subjected to conditions of imposed flux. Bairi et al. [2] by using the radiosity technique, note that in a diode cavity, presence of radiation incoming from the interfaces strongly affects the natural convection in a non-participating medium, and may reduce it substantially for particular edge angles. The case of participating semitransparent media confined in cartesian closed cavities has been examined for several decades: Tan and Howell [3] studied the influence of radiation combined to convection in an active fluid inside a square cavity by discretising the exact equations of the radiative transfer. Cartesian geometries are mostly studied with discrete ordinates methods (DOM), Monte-Carlo or ray tracing methods to take into account the directional nature of radiation [4-6], often combined with finite volumes methods (FVM) to deal with the space discretisation. Han and Baek [7] used the FVM for solving the radiative transfer equation to look at the influence of radiation combined to convection in a rectangular cavity with radiant surfaces, and observed that radiation plays a significant role in developing the fluid dynamic. The DOM is suitable and easily implementable in the case of diffusely reflecting surfaces in rectangular types cavities discretized with regular structured grids, and significant improvements have been brought to this technique by removing partially the ray effects. Mishra et al. [8] proposed an improved DOM by choosing the quadrature angles and weights leading to accurate results, which partially removes the ray effects. The DOM has also been extended to spherical geometries and used for analysing radiative transfer in cavities with specularly reflecting walls. Li et al. [9] combined a conventional DOM and a 
Chebyshev polynomial expansion for the space dependence in the treatment of the radiative transfer equation (RTE) to deal with the combined conduction-radiation transfer inside a spherical participating medium in a spherical annulus, and managed to obtain accurate results for the total heat flux compared to an exact method, especially for moderately or highly absorbing media. Le Hardy et al. examined an original way to discretize the specular boundary conditions by calculating a partition ratio coefficients for the reflected solid angles combined with an adequate weak formulation in the frame of the DOM [10]. However, such techniques suffer from a lack of accuracy when dealing with internal media of small absorption coefficients, or as soon as the geometry is more complex. Meshless-like methods have also been developed to take into account radiation in closed cavities of complex shapes bounded by diffusely reflecting surfaces to evaluate the radiative field by applying a specific treatment to the space derivation operator of the radiative transfer equation [11], which produces accurate results in media of moderate to strong absorption coefficients [12]. Semi-analytical methods have been proposed to deal with pure radiation inside cavities of complex non-rectangular shapes, to avoid false diffusion and ray effects of the DOM and deal with weakly absorbing media, either with or without reflecting walls [13-14], or both conduction and radiation [15]. The elegant class of semi-analytical methods belongs to the alternative works proposed by Altaç et al. [16] who extended the pioneering study of Crosbie et al. [17] by introducing a set of special analytical functions, which produces highly accurate values of the incident radiation and radiative flux inside a scattering absorbing medium bounded by a two-dimensional cavity with cold surfaces. This partially analytical method is particularly appropriate to obtain the radiative field in complex geometries with reflecting surfaces, the analytical part being not limited to geometries of regular shapes. It has been successfully applied to cylindrical and spherical annuli with diffuse or specular reflector surfaces [18].

Although several complex geometries have been numerically studied with various techniques such as meshless methods [12] when radiation holds in the cavity, the case of solid obstacles inside a closed cavity with a radiant medium has not enhanced an abundant literature. Conductive and/or convective heat transfer inside closed cavities with internal obstacles has been investigated without radiation: Ul Haq et al. [19] studied the heat transfer inside a liquid through a triangular cavity with a heated cylindrical obstacle. Mezrhab et al. [20] used the FVM to discuss the influence of radiant surfaces on the convection through a transparent medium inside a square cavity with an inner centered square obstacle. The influence of a participating radiant medium has been studied by Coelho et al. [21] who examined the radiative transfer in a rectangular cavity with one-dimensional of zero thickness obstacles of finite length, and compared the zones method, discrete transfer and DOM techniques efficiency in the calculation of the heat fluxes on the cavity's walls. Sakami et al. [22] used a modified DOM to analyse the radiative transfer inside cavities of various shapes meshed by structured grids and containing an inner opaque black square obstacle, to calculate the radiative flux on the walls of the cavity when the internal semi-transparent medium is isothermal. Wang and Liu [23] applied a Galerkin finite elements method to solve the RTE in a two-dimensional cavity with internal obstacles for unstructured grids to avoid the strong ray effects. In the previous works, although the semi-transparent medium is both emitting-absorbing and scattering, it is generally isothermal at an imposed constant temperature and no real heat transfer occurs, the main problem being in the effective resolution of the RTE and applying it to the flux calculation. We recently examined the case of a centred square opaque obstacle inside a square cavity containing an emittingabsorbing medium at radiative equilibrium by using an exact analytical method [24] which proved its robustness, both at radiative equilibrium and in situation of radiation/conduction coupling [25]. Although quite complex to implement due to difficult calculations, it is to our best knowledge the only one method which efficiently points out the major rule of the ratio $\frac{h}{H}$ in the radiative behaviour of the whole device and produces smooth accurate results for the different values of the previous ratio. 
Up to now, a very few literature survey is available on the case of a reflecting surfaces obstacle surrounded by a semi-transparent emitting-absorbing medium contained inside a cavity with reflecting surfaces. The case of a centred spherical obstacle inside a spherical cavity [18] has strongly put in evidence that reflecting surfaces could severely affect the radiative field inside the medium, and it is highly probable that such a conclusion may arise inside a complex device of a reflecting square obstacle inside a reflecting cavity. Due to the major difficulty of treating the radiative transfer in reflecting cavities of complex non-cartesian shapes, as pointed out in [14], we shall hereafter limit our investigations first to the determination of the radiosity temperatures on both the obstacle and cavity surfaces when all the surfaces of the global device are purely diffusely reflectors. To our best knowledge, no study is available concerning the radiative behaviour of a semi-transparent medium enclosed in a rectangular cavity with reflecting surfaces and a rectangular opaque and reflecting obstacle inside the cavity: then our work is new from a radiative point of view. The radiosity temperatures are nevertheless a good indicator of the global radiative behaviour of the internal medium since they act like non-isothermal black surfaces.

The paper is organized as follows: in section II we develop the exact expressions of the local radiosity temperatures on the reflecting/emitting boundary surfaces, different from the real temperatures due to the presence of diffuse reflection. In section III we explain the angular and spatial discretisation of the useful integrals. Finally we present some numerical applications in section IV and end the work by a short conclusion.

\section{II - PHYSICAL AND MATHEMATICAL MODELLING}

One examines a two-dimensional square cavity with an internal non participating square opaque obstacle at a centered position, as described in Fig. 1. The cavity is filled with a non-isothermal absorbing-emitting gray semi-transparent medium characterized by its absorption coefficient $\kappa$. The boundary surfaces of the cavity and of the internal obstacle are opaque and diffusely reflecting, at imposed temperatures.

\section{II-1. Geometry}

The square section is divided into isothermal cells of depth $\Delta x$ including 5 zero depth point cells on each surface of the cavity and the obstacle at $x \in\left\{0, \frac{H-h}{2}, \frac{H}{2}, \frac{H+h}{2}, H\right\}$ which do not interfere in the determination of the radiosity temperatures, but where the radiosity temperatures can be determined as soon as the temperatures in the non-zero depth cells have been calculated.
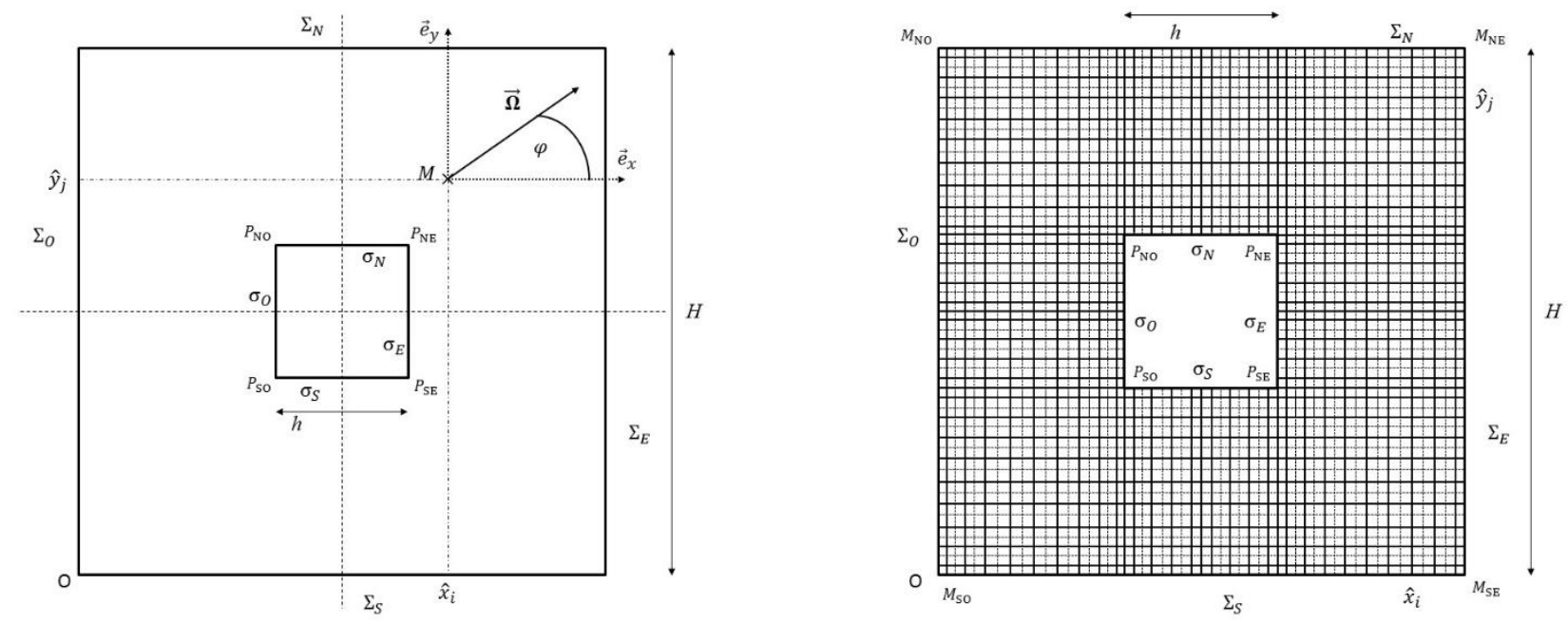

Figure 1: Description of the cavity's geometry and the discrete system

The coordinates of any calculation point are given following a numerical sequence defined by: 
$* x_{1}=0$

$* x_{k}=\left(k-\frac{3}{2}\right) \Delta x$

$$
2 \leq k \leq M-1
$$

$* x_{M}=(M-2) \Delta x=\frac{H-h}{2}$

$* x_{M-1+k}=x_{M}+\left(k-\frac{3}{2}\right) \Delta x$

$2 \leq k \leq P-1$

* $x_{M+P-1}=(M+P-4) \Delta x=\frac{H}{2}$

$* x_{M+P-2+k}=x_{M+P-1}+\left(k-\frac{3}{2}\right) \Delta x \quad 2 \leq k \leq P-1$

* $x_{M+2 P-2}=(M+2 P-6) \Delta x=\frac{H+h}{2}$

$* x_{M+2 P-3+k}=x_{M+2 P-2}+\left(k-\frac{3}{2}\right) \Delta x \quad 2 \leq k \leq M-1$

$* x_{2 M+2 P-3}=2(M+P-4) \Delta x=H$

The scheme generates a regular grid containing in each direction $N_{t}=2 M+2 P-3$ cells, five of which being punctual $\left(x_{1}, x_{M}, x_{M+P-1}, x_{M+2 P-2}\right.$ and $\left.x_{2 M+2 P-3}\right)$, the cell $x_{M+P-1}$ in the centre of a boundary surface, with $2(M-2)$ internal cells in the physical domain $] 0, \frac{H-h}{2}[\cup] \frac{H+h}{2}, H[$ and $2(P-2)$ internal cells (centre excluded) in the domain $] \frac{H-h}{2}, \frac{H+h}{2}\left[\right.$, each point $M_{i j}$ centred in the square cell labelled $(i, j)$ of size $\Delta x \Delta y \equiv \Delta x^{2}$ being defined as $M_{i j}\left(\hat{x}_{i}, \hat{y}_{j}\right)$, with:

$$
\begin{aligned}
(i, j) \in\{1, M\} & \times\{1,2 M+2 P-3\} \cup\{M+1, M+2 P-3\} \times\{1, M\} \cup\{M+1, M+2 P-3\} \\
& \times\{M+2 P-2,2 M+2 P-3\} \cup\{M+2 P-2,2 M+2 P-3\} \times\{1,2 M+2 P-3\}
\end{aligned}
$$

\section{II-2. Definition and determination of the radiosity temperatures at the boundary surfaces}

The incident radiative flux on a surface writes:

$$
q^{-}=\int_{\Omega n<0} I^{\leftarrow}(\Omega)|\Omega n| d \Omega
$$

where $I^{\leftarrow}(\boldsymbol{\Omega})$ stands for the radiative incoming intensity on the boundary surface and $\boldsymbol{n}$ its inward unit normal vector. When the boundary is diffusely reflecting, the outgoing radiative intensity $I^{\rightarrow}$ leaving the surface is deduced from the diffuse reflexion law:

$$
I^{\rightarrow}=\varepsilon_{W} \frac{\sigma T_{w}^{4}}{\pi}+\frac{\rho_{W}}{\pi} \int_{\boldsymbol{\Omega n}<0} I^{\leftarrow}(\boldsymbol{\Omega})|\boldsymbol{\Omega n}| d \boldsymbol{\Omega}
$$

where $\varepsilon_{W}$ and $\rho_{W}$ are the emissivity and the reflexion factor of the boundary wall, with $\rho_{W}=1-\varepsilon_{W}$. The incoming radiative intensity $I^{\leftarrow}(\Omega)$ on a boundary surface for a given direction $\Omega$ results from the whole radiant contribution of the surrounding emitting-absorbing semi-transparent medium and the proper contribution of the other system's surfaces. The contributing intensity of the surrounding medium is obtained by solving the radiative transfer equation (RTE), here written for a gray non-scattering nonisothermal medium:

$$
\frac{d I}{d s}(s, \Omega)+\kappa I(s, \Omega)=\frac{\kappa \sigma T^{4}}{\pi}(s)
$$


where the differential term stands for $\frac{d I}{d s}(s, \boldsymbol{\Omega})=\boldsymbol{\Omega} \cdot \operatorname{grad}[I(s, \boldsymbol{\Omega})]$, the dot noting the usual scalar product and $s$ the local curvilinear abscissa. Under this one-dimensional differential form, the solution of (3) is immediately given by:

$$
I(s, \boldsymbol{\Omega})=I\left(s_{f}, \boldsymbol{\Omega}\right) e^{-\kappa\left(s_{f}-s\right)}+\frac{\kappa \sigma}{\pi} \int_{s^{\prime}=s}^{s_{f}} T^{4}\left(s^{\prime}\right) e^{-\kappa\left(s^{\prime}-s\right)} d s^{\prime}
$$

This fundamental expression will be rewritten under an adequate form depending on the geometry, which shall be detailed in the next subsection.

Since each surface is perfectly diffusely reflecting, the outgoing intensity does not depend on the outgoing direction, which defines a radiosity temperature $T_{R}$ as:

$$
\sigma T_{R}^{4}=\varepsilon_{W} \sigma T_{w}^{4}+\rho_{W} \int_{\boldsymbol{\Omega n}<0} I^{\leftarrow}(\boldsymbol{\Omega})|\boldsymbol{\Omega n}| d \Omega
$$

\section{II-2.1: Exact expressions of the obstacle's surfaces radiosity temperatures}

We shall now develop the whole calculation for the eastern surface $\sigma_{E}$ of the internal obstacle. To simplify the notations, we introduce the three fundamental optical depths as $\tau_{0}=\frac{\kappa(H-h)}{2}$ and $\tau_{0}^{\prime}=\kappa H$, with $\tau_{0}^{\prime}-\tau_{0}=\frac{\kappa(H+h)}{2}=\tau_{0}^{\prime \prime}$.

For a given point $M_{\sigma_{E}}\left(\frac{H+h}{2}, \frac{H-h}{2} \leq \hat{y}_{k} \leq \frac{H+h}{2}\right)$ on $\sigma_{E}$, the incoming radiative flux from its surrounding environment is obtained from:

$$
\begin{aligned}
\int_{\cos \varphi<0} I^{\leftarrow}\left(\sigma_{E}, \theta, \varphi\right)|\cos \varphi| d \varphi \\
\quad=\int_{\varphi=0}^{\xi_{E}^{+}} I^{\rightarrow}\left(\Sigma_{E}^{+}\right) \cos \varphi d \varphi+\int_{\varphi=\xi_{E}^{+}}^{\frac{\pi}{2}} I^{\rightarrow}\left(\Sigma_{N}\right) \cos \varphi d \varphi+\int_{\varphi=0}^{2 \pi-\xi_{E}^{-}} I^{\rightarrow}\left(\Sigma_{S}\right) \cos \varphi d \varphi \\
\quad+\int_{\varphi=2 \pi-\xi_{E}^{-}}^{2 \pi} I^{\rightarrow}\left(\Sigma_{E}^{-}\right) \cos \varphi d \varphi
\end{aligned}
$$

since the propagation angle $\boldsymbol{\Omega}$ writes $\boldsymbol{\Omega}=\left(\begin{array}{c}\cos \varphi \sin \theta \\ \sin \varphi \sin \theta \\ \cos \theta\end{array}\right)$ in the natural basis $\left(\boldsymbol{e}_{\boldsymbol{x}}, \boldsymbol{e}_{\boldsymbol{y}}, \boldsymbol{e}_{z}\right)$.

In the previous expression (6) the limiting two angles $\xi_{E}^{+}$and $\xi_{E}^{-}$are defined by $\xi_{E}^{+}=\tan ^{-1}\left[\frac{2\left(H-\hat{y}_{k}\right)}{H-h}\right]$, $\xi_{E}^{-}=\tan ^{-1}\left(\frac{2 \hat{y}_{k}}{H-h}\right)$, and $I^{\leftarrow}\left(\sigma_{E}, \theta, \varphi\right)$ represents the incident radiation on $\sigma_{E}$, the $I^{\rightarrow}$ being the outgoing intensities from a surface cavity up to the point $M_{\sigma_{E}}$.

The intensity $I^{\rightarrow}\left(\Sigma_{E}^{+}\right)$incoming from the upper eastern surface of the cavity to the eastern obstacle' surface for any direction in the figure's plane is deduced from Eq. (4) in the general case of a nonisothermal medium:

$$
\begin{aligned}
I^{\rightarrow}\left(\Sigma_{E}^{+}\right)=\frac{\sigma}{\pi} & T_{R E}^{4}\left(\tau_{k}^{+}+\tau_{0} \tan \varphi\right) e^{-\frac{\tau_{0}}{\cos \varphi}} \\
& \quad+\frac{\sigma}{\pi \cos \varphi} \int_{\tau_{u}^{+}=\tau_{0}^{\prime \prime}}^{\tau \prime_{0}} T^{4}\left[\tau_{u}^{+}, \tau_{k}^{+}+\left(\hat{u}-\tau_{0}{ }_{0}\right) \tan \varphi\right] e^{-\frac{\tau_{u}^{+}-\tau_{0}}{\cos \varphi}} d \tau_{u}^{+}
\end{aligned}
$$


since for a straight line in the direction $\boldsymbol{\Omega}$, the differential element $d s$ of Eq. (4) is given by $d s=\frac{d \widehat{u}}{\cos \varphi}$ where $\hat{u}$ represents the coordinate along the axis $\boldsymbol{e}_{\boldsymbol{x}}$, any point in the plane $\left(\boldsymbol{e}_{\boldsymbol{x}}, \boldsymbol{e}_{\boldsymbol{y}}\right)$ on the line $\boldsymbol{\Omega}$ between $\sigma_{E}$ and the surface $\Sigma_{E}^{+}$being characterised by its local coordinates $\hat{u}$ and $\hat{y}_{k}+\left(\hat{u}-\frac{H+h}{2}\right) \tan \varphi$. The local optical depth of Eq. (7) are noted $\tau_{k}^{+}=\kappa \hat{y}_{k}$ and $\tau_{u}^{+}=\kappa \hat{u}$, whence $\tau_{u}^{+}-\left(\tau^{\prime}{ }_{0}-\tau_{0}\right)=\tau_{0}-\tau_{u}^{-}$where $\tau_{u}^{-}=\kappa(H-\hat{u})=\tau^{\prime}{ }_{0}-\tau_{u}^{+}$

In the previous expression, $T_{R E}^{4}$ is the radiosity temperature of the cavity's eastern boundary surface, depending on the space location on the surface. Taking into account all the propagation directions not only located in the figure's plane leads to the integrated intensity expressed by, with the $K i_{n}$ BickleyNaylor functions [26]:

$$
\begin{aligned}
& I^{\rightarrow}\left(\Sigma_{E}^{+}\right)=\frac{\sigma}{\pi} T_{R E}^{4}\left(\tau_{k}^{+}+\tau_{0} \tan \varphi\right) K i_{3}\left(\frac{\tau_{0}}{\cos \varphi}\right) \\
& \quad+\frac{\sigma}{\pi \cos \varphi} \int_{\tau_{u}^{+}=\tau_{0}^{\prime \prime}}^{\tau_{0}^{\prime}} T^{4}\left[\tau_{u}^{+}, \tau_{k}^{+}+\left(\tau_{u}^{+}-\tau^{\prime \prime}{ }_{0}\right) \tan \varphi\right] K i_{2}\left(\frac{\tau_{0}-\tau_{u}^{-}}{\cos \varphi}\right) d \tau_{u}^{+}
\end{aligned}
$$

Similarly, noting $\tau_{k}^{-}=\kappa\left(H-\hat{y}_{k}\right)$, the contributing part of the cavity's northern boundary surfaces leads to the following expression:

$$
\begin{aligned}
I^{\rightarrow}\left(\Sigma_{N}\right)=\frac{\sigma}{\pi} T_{R N}^{4}\left(\tau^{\prime \prime}{ }_{0}+\frac{\tau_{k}^{-}}{\tan \varphi}\right) K i_{3}\left(\frac{\tau_{k}^{-}}{\sin \varphi}\right) \\
\quad+\frac{\sigma}{\pi \sin \varphi} \int_{\tau_{u}^{+}=\tau_{k}^{+}}^{\tau_{0}} T^{4}\left(\tau^{\prime \prime}{ }_{0}+\frac{\tau_{u}^{+}-\tau_{k}^{+}}{\tan \varphi}, \tau_{u}^{+}\right) K i_{2}\left(\frac{\tau_{u}^{+}-\tau_{k}^{+}}{\sin \varphi}\right) d \tau_{u}^{+}
\end{aligned}
$$

whence it comes for the southern boundary surface's contribution:

$$
\begin{aligned}
I^{\rightarrow}\left(\Sigma_{S}\right)=\frac{\sigma}{\pi} T_{R S}^{4}\left(\tau^{\prime \prime}{ }_{0}+\frac{\tau_{k}^{+}}{|\tan \varphi|}\right) K i_{3}\left(\frac{\tau_{k}^{+}}{|\sin \varphi|}\right) \\
\quad+\frac{\sigma}{\pi|\sin \varphi|} \int_{\tau_{u}^{+}=0}^{\tau_{k}^{+}} T^{4}\left(\tau^{\prime \prime}{ }_{0}+\frac{\tau_{u}^{-}-\tau_{k}^{-}}{|\tan \varphi|}, \tau_{u}^{+}\right) K i_{2}\left(\frac{\tau_{u}^{-}-\tau_{k}^{-}}{|\sin \varphi|}\right) d \tau_{u}^{+}
\end{aligned}
$$

Finally, the lower eastern surface of the cavity's contribution is deduced from (9):

$$
\begin{aligned}
I^{\rightarrow}\left(\Sigma_{E}^{-}\right)=\frac{\sigma}{\pi} & T_{R E}^{4}\left(\tau_{k}^{+}-\tau_{0} \tan \varphi\right) K i_{3}\left(\frac{\tau_{0}}{\cos \varphi}\right) \\
& +\frac{\sigma}{\pi \cos \varphi} \int_{\tau_{u}^{+}=\tau_{0}^{\prime \prime}}^{\tau_{0}} T^{4}\left[\tau_{u}^{+}, \tau_{k}^{+}-\left(\tau_{0}-\tau_{u}^{-}\right) \tan \varphi\right] K i_{2}\left(\frac{\tau_{0}-\tau_{u}^{-}}{\cos \varphi}\right) d \tau_{u}^{+}
\end{aligned}
$$

From which the global expression of the eastern surface of the obstacle radiosity temperature writes: 


$$
\begin{aligned}
\frac{\pi\left[T_{R \sigma_{E}}^{4}\left(\hat{y}_{k}\right)-\varepsilon_{\sigma_{E}} T_{\sigma_{E}}^{4}\right]}{2 \rho_{\sigma_{E}}} & \\
& =\int_{\varphi=0}^{\xi_{E}^{+}} T_{R E}^{4}\left(\tau_{k}^{+}+\tau_{0} \tan \varphi\right) K i_{3}\left(\frac{\tau_{0}}{\cos \varphi}\right) \cos \varphi d \varphi \\
& +\int_{\varphi=\xi_{E}^{+}}^{\frac{\pi}{2}} T_{R N}^{4}\left(\tau^{\prime \prime}{ }_{0}+\frac{\tau_{k}^{-}}{\tan \varphi}\right) K i_{3}\left(\frac{\tau_{k}^{-}}{\sin \varphi}\right) \cos \varphi d \varphi \\
& +\int_{\varphi=\frac{3 \pi}{2}}^{2 \pi-\xi_{E}^{-}} T_{R S}^{4}\left(\tau^{\prime \prime}{ }_{0}+\frac{\tau_{k}^{+}}{|\tan \varphi|}\right) K i_{3}\left(\frac{\tau_{k}^{+}}{|\sin \varphi|}\right) \cos \varphi d \varphi \\
& +\int_{\varphi=2 \pi-\xi_{E}^{-}}^{2 \pi} T_{R E}^{4}\left(\tau_{k}^{+}-\tau_{0} \tan \varphi\right) K i_{3}\left(\frac{\tau_{0}}{\cos \varphi}\right) \cos \varphi d \varphi+\kappa \aleph_{\sigma_{E}}
\end{aligned}
$$

where the global internal contribution $\aleph_{\sigma_{E}}$ due to the non-isothermal emitting-absorbing semi-transparent medium itself is expressed by:

$$
\begin{aligned}
& \kappa \aleph_{\sigma_{E}}=\int_{\varphi=0}^{\xi_{E}^{+}} \int_{\tau_{u}^{+}=\frac{H+h}{2}}^{\tau^{\prime}} T^{4}\left[\tau_{u}^{+}, \tau_{k}^{+}+\left(\tau_{0}-\tau_{u}^{-}\right) \tan \varphi\right] K i_{2}\left(\frac{\tau_{0}-\tau_{u}^{-}}{\cos \varphi}\right) d \tau_{u}^{+} d \varphi \\
&+\int_{\varphi=\xi_{E}^{+}}^{\frac{\pi}{2}} \int_{\tau_{u}^{+}=\tau_{k}^{+}}^{\tau_{0}} T^{4}\left(\tau^{\prime \prime}{ }_{0}+\frac{\tau_{u}^{+}-\tau_{k}^{+}}{\tan \varphi}, \hat{u}\right) K i_{2}\left(\frac{\tau_{u}^{+}-\tau_{k}^{+}}{\sin \varphi}\right) d \tau_{u}^{+} \frac{d \varphi}{\tan \varphi} \\
&+\int_{\varphi=\frac{3 \pi}{2}}^{2 \pi-\xi_{E}^{-}} \int_{\tau_{u}^{+}=0}^{\tau_{k}^{+}} T^{4}\left(\tau^{\prime \prime}{ }_{0}+\frac{\tau_{u}^{-}-\tau_{k}^{-}}{|\tan \varphi|}, \hat{u}\right) K i_{2}\left(\frac{\tau_{u}^{-}-\tau_{k}^{-}}{|\sin \varphi|}\right) d \tau_{u}^{+} \frac{d \varphi}{|\tan \varphi|} \\
&+\int_{\varphi=2 \pi-\xi_{E}^{-}}^{2 \pi} \int_{\tau_{u}^{+}=\tau_{0}{ }_{0}}^{\tau^{\prime}{ }_{0}} T^{4}\left[\tau_{u}^{+}, \tau_{k}^{+}-\left(\tau_{0}-\tau_{u}^{-}\right)|\tan \varphi|\right] K i_{2}\left(\frac{\tau_{0}-\tau_{u}^{-}}{\cos \varphi}\right) d \tau_{u}^{+} d \varphi
\end{aligned}
$$

Applying the variable changes $\varphi \leftrightarrow \frac{\pi}{2}-\varphi$ for the northern contribution, $\varphi \leftrightarrow \varphi-\frac{3 \pi}{2}$ for the southern one and $\varphi \leftrightarrow 2 \pi-\varphi$ for the lower part of the eastern cavity's surface leads to:

$$
\begin{aligned}
\frac{\pi\left[T_{R \sigma_{E}}^{4}\left(\hat{y}_{k}\right)-\varepsilon_{\sigma_{E}} T_{\sigma_{E}}^{4}\right]}{2 \rho_{\sigma_{E}}} & \\
& =\int_{\varphi=0}^{\xi_{E}^{+}} T_{R E}^{4}\left(\tau_{k}^{+}+\tau_{0} \tan \varphi\right) K i_{3}\left(\frac{\tau_{0}}{\cos \varphi}\right) \cos \varphi d \varphi \\
& +\int_{\varphi=0}^{\xi_{E}^{-}} T_{R E}^{4}\left(\tau_{k}^{+}-\tau_{0} \tan \varphi\right) K i_{3}\left(\frac{\tau_{0}}{\cos \varphi}\right) \cos \varphi d \varphi \\
& +\int_{\varphi=0}^{\xi_{N}^{+}} T_{R N}^{4}\left(\tau^{\prime \prime}{ }_{0}+\tau_{k}^{-} \tan \varphi\right) K i_{3}\left(\frac{\tau_{k}^{-}}{\cos \varphi}\right) \sin \varphi d \varphi \\
& +\int_{\varphi=0}^{\xi_{S}^{+}} T_{R S}^{4}\left(\tau^{\prime \prime}{ }_{0}+\tau_{k}^{+} \tan \varphi\right) K i_{3}\left(\frac{\tau_{k}^{+}}{\cos \varphi}\right) \sin \varphi d \varphi+\kappa \aleph_{\sigma_{E}}
\end{aligned}
$$

where the angles $\xi_{N}^{+}$and $\xi_{S}^{+}$write $\xi_{N}^{+}=\tan ^{-1}\left[\frac{H-h}{2\left(H-\hat{y}_{k}\right)}\right]=\frac{\pi}{2}-\xi_{E}^{+}$and $\xi_{S}^{+}=\tan ^{-1}\left(\frac{H-h}{2 \hat{y}_{k}}\right)=\frac{\pi}{2}-\xi_{E}^{-}$.

Note that in a square cavity, $\hat{y}_{k} \equiv \hat{x}_{k}$ and we shall hereafter also note $\xi_{E}^{+} \equiv \tan ^{-1}\left[\frac{2\left(H-\hat{x}_{k}\right)}{H-h}\right]$ and equivalently for the three other angles $\xi_{E}^{-}, \xi_{N}^{+}$and $\xi_{S}^{+}$. Similarly the internal contribution $\aleph_{\sigma_{E}}$ writes: 


$$
\begin{aligned}
\kappa \aleph_{\sigma_{E}}=\int_{\tau_{u}^{+}=0}^{\tau_{0}} & \left\{\int_{\varphi=0}^{\xi_{E}^{+}} T^{4}\left(\tau_{u}^{+}+\tau^{\prime \prime}{ }_{0}, \tau_{k}^{+}+\tau_{u}^{+} \tan \varphi\right) K i_{2}\left(\frac{\tau_{u}^{+}}{\cos \varphi}\right) d \varphi\right. \\
& \left.+\int_{\varphi=0}^{\xi_{E}} T^{4}\left(\tau_{u}^{+}+\tau^{\prime \prime}{ }_{0}, \tau_{k}^{+}-\tau_{u}^{+} \tan \varphi\right) K i_{2}\left(\frac{\tau_{u}^{+}}{\cos \varphi}\right) d \varphi\right\} d \tau_{u}^{+} \\
& +\int_{\tau_{u}^{+}=0}^{\tau_{k}^{-}} \int_{\varphi=0}^{\xi_{N}^{+}} T^{4}\left(\tau^{\prime \prime}{ }_{0}+\tau_{u}^{+} \tan \varphi, \tau_{k}^{+}+\tau_{u}^{+}\right) K i_{2}\left(\frac{\tau_{u}^{+}}{\cos \varphi}\right) \tan \varphi d \varphi d \tau_{u}^{+} \\
& +\int_{\tau_{u}^{+}=0}^{\tau_{k}^{+}} \int_{\varphi=0}^{\xi_{S}^{+}} T^{4}\left(\tau^{\prime \prime}{ }_{0}+\tau_{u}^{+} \tan \varphi, \tau_{u}^{-}-\tau_{k}^{-}\right) K i_{2}\left(\frac{\tau_{u}^{+}}{\cos \varphi}\right) \tan \varphi d \varphi d \tau_{u}^{+}
\end{aligned}
$$

For isothermal media, the constant internal temperature $T$ can be dropped from the integrals, leading to:

$$
\frac{\kappa \aleph_{\sigma_{E}}}{T^{4}}=\frac{\pi}{2}-B i s_{3}\left(\tau_{0}, \xi_{E}^{+}\right)-B i s_{3}\left(\tau_{0}, \xi_{E}^{-}\right)-C i s_{3}\left(\tau_{k}^{-}, \xi_{N}^{+}\right)-C i s_{3}\left(\tau_{k}^{+}, \xi_{S}^{+}\right)
$$

where the functions $B i s_{3}$ and $\mathrm{Cis}_{3}$ [27] are defined by $\operatorname{Bis}_{n}(x, \theta)=\int_{\varphi=0}^{\theta} K i_{n}\left(\frac{x}{\cos \varphi}\right)(\cos \varphi)^{n-2} d \varphi$ and $\operatorname{Cis}_{n}(x, \theta)=\int_{\varphi=0}^{\theta} K i_{n}\left(\frac{x}{\cos \varphi}\right)(\cos \varphi)^{n-3} \sin \varphi d \varphi$.

The three other radiosity temperatures on the obstacle's surfaces are obtained from formal substitutions $\hat{x} \leftrightarrow H-\hat{x}$ for the western one, $\hat{y} \leftrightarrow \hat{x}$ for the northern one and $\hat{y} \leftrightarrow H-\hat{y}$ for the southern one from the northern contribution, leading to similar expressions which are reported in the Annex 1.

When the obstacle's surfaces radiosity temperatures are obtained, it is possible to determine the cavity's surfaces radiosity temperatures in a similar way, which is the aim of the subsection.

\section{II-2.2: Exact expressions of the cavity's surfaces radiosity temperatures}

As for the obstacle, we pay attention to the eastern cavity's surface radiosity temperature determination. Let us name $P_{\mathrm{NO}}, P_{\mathrm{NE}}, P_{\mathrm{SE}}$ and $P_{\mathrm{SO}}$ the four obstacle's edge points and define a calculation point $M_{k}$ characterized by its ordinate $\hat{y}_{k}$.

We shall examine first points for which $\hat{y}_{k} \leq \frac{H-h}{2}$, as illustrated in Fig. 2:
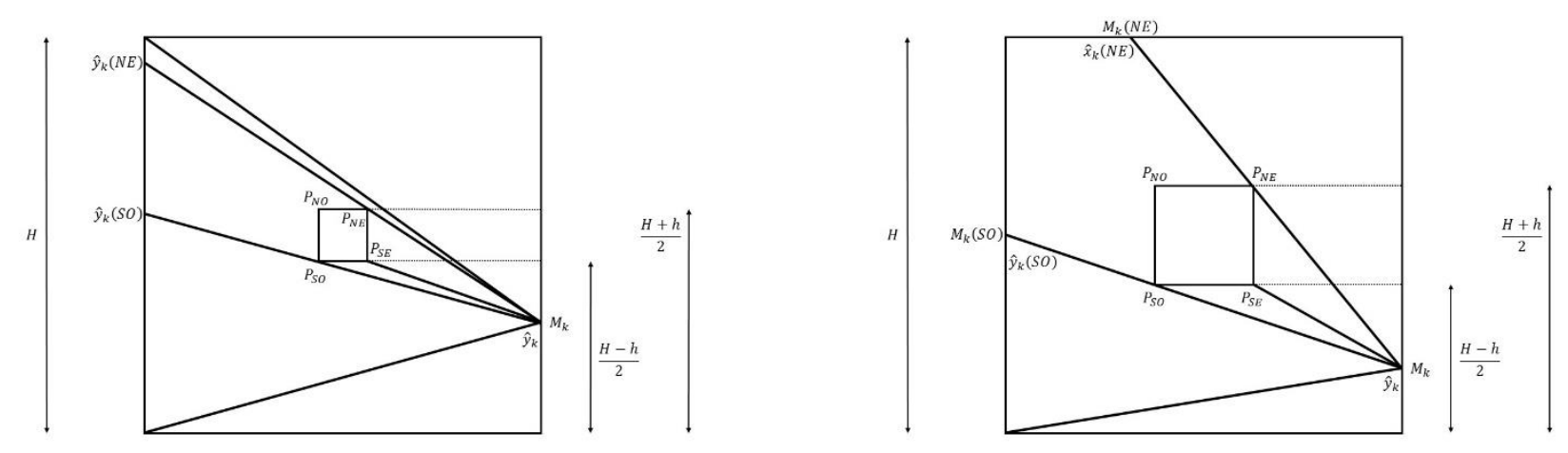

(a): $\frac{h}{H} \leq \sqrt{5}-2$ and $\frac{2 h H}{H+h}<\hat{y}_{k} \leq \frac{H-h}{2}$

(b): $\left(\frac{h}{H} \leq \sqrt{5}-2\right.$ and $\left.0 \leq \hat{y}_{k} \leq \frac{2 h H}{H+h}\right)$ or $\left(\frac{h}{H}>\sqrt{5}-2\right.$ and $\left.0 \leq \hat{y}_{k} \leq \frac{H-h}{2}\right)$

Figure 2: Determination of the eastern cavity's surface radiosity temperature when $\hat{y}_{k} \leq \frac{H-h}{2}$ 
The point $M_{k}(S O)$ always located both on the western cavity's surface $\Sigma_{O}$ and on the straight line passing through the two points $P_{\mathrm{SO}}$ and $M_{k}$ is characterized by its ordinate $\hat{y}_{k}(S O)=\frac{(H-h)\left(H-\hat{y}_{k}\right)}{H+h}$.

Similarly, the abscissa of the point $M_{k}(N E)$ on $\Sigma_{N}$ and belonging to the straight line $\left(P_{\mathrm{NE}}, M_{k}\right)$ is defined by $\hat{x}_{k}(N E)=\frac{2 h H-(H+h) \hat{y}_{k}}{H+h-2 \hat{y}_{k}}$ which may be negative if $\hat{y}_{k}>\frac{2 h H}{H+h}$, case implying that the point $M_{k}(N E)$ cannot be on the northern surface but always on the western one. The simultaneous condition $\hat{y}_{k} \leq \frac{H-h}{2}$ leads to $\frac{h}{H} \leq \sqrt{5}-2$.

Then if $\frac{h}{H} \leq \sqrt{5}-2$ and $\frac{2 h H}{H+h}<\hat{y}_{k} \leq \frac{H-h}{2}$, the point $M_{k}(N E)$ belongs to $\Sigma_{O}$ with $\hat{y}_{k}(N E)=\frac{(H+h)\left(H-\hat{y}_{k}\right)}{H-h}$, whence the calculation point $M_{k}$ sees the whole northern cavity's surface, the total southern cavity's surface and a small piece of the $\Sigma_{O}$ delimited on the segment $y \in\left[0, \frac{(H-h)\left(H-\hat{y}_{k}\right)}{H+h}\right] \cup\left[\frac{(H+h)\left(H-\hat{y}_{k}\right)}{H-h}, H\right]$.

In this case, the local eastern radiosity temperature writes:

$$
\begin{aligned}
\frac{\sigma T_{R E}^{4}\left(\hat{y}_{k}\right)-\varepsilon_{E} \sigma T_{E}^{4}}{2 \rho_{E}} & \\
& =\int_{\theta=0}^{\frac{\pi}{2}}\left[\int_{\varphi=\frac{\pi}{2}}^{\pi-\alpha_{O}^{+}} I_{N}^{+}(\theta, \varphi)|\cos \phi| d \varphi+\int_{\varphi=\pi-\alpha_{O}^{+}}^{\pi-\alpha_{\sigma_{E}}^{+}} I_{O}^{+}(\theta, \varphi)|\cos \varphi| d \varphi\right. \\
& +\int_{\varphi=\pi-\alpha_{\sigma_{E}}^{+}}^{\pi-\alpha_{\sigma_{E}}^{+}} I_{\sigma_{E}}^{+}(\theta, \varphi)|\cos \varphi| d \varphi+\int_{\varphi=\pi-\alpha_{\sigma_{E}}^{-}}^{\pi-\alpha_{O 1}^{+}} I_{\sigma_{S}}^{+}(\theta, \varphi)|\cos \varphi| d \varphi \\
& \left.+\int_{\varphi=\pi-\alpha_{O 1}^{+}}^{\pi+\alpha_{O}^{-}} I_{O}^{+}(\theta, \varphi)|\cos \varphi| d \varphi+\int_{\varphi=\pi+\alpha_{O}^{-}}^{\frac{3 \pi}{2}} I_{S}^{+}(\theta, \varphi)|\cos \varphi| d \varphi\right] \sin ^{2} \theta d \theta
\end{aligned}
$$

where the $I_{N, O, S, \sigma_{E}, \sigma_{S}}^{+}$are the leaving intensities from the considered surfaces, taking into account the internal contribution up to the calculation point $M_{k}$.

The different angles in the integrals of (17) are defined by $\alpha_{O}^{+}=\tan ^{-1}\left(\frac{H-\hat{y}_{k}}{H}\right), \alpha_{\sigma_{E}}^{+}=\tan ^{-1}\left(\frac{H+h-2 \hat{y}_{k}}{H-h}\right)$, $\alpha_{\sigma_{E}}^{-}=\tan ^{-1}\left(\frac{H-h-2 \hat{y}_{k}}{H-h}\right), \alpha_{O 1}^{+}=\tan ^{-1}\left(\frac{H-h-2 \hat{y}_{k}}{H+h}\right)$ and $\alpha_{O}^{-}=\tan ^{-1}\left(\frac{\hat{y}_{k}}{H}\right)$.

For a propagation direction $(\theta, \varphi)$ such that $\varphi \in\left[\frac{\pi}{2}, \pi-\alpha_{O}^{+}\right]$, the intensity leaving the northern cavity's boundary and arriving at $M_{k}$ writes, with $\alpha_{N}^{+}=\frac{\pi}{2}-\alpha_{O}^{+}=\tan ^{-1}\left(\frac{H}{H-\hat{y}_{k}}\right)$ :

$$
\begin{aligned}
I_{N}^{+\rightarrow M_{k}}(\theta, \varphi)= & \frac{\sigma}{\pi} T_{R N}^{4}\left(\tau^{\prime}{ }_{0}-\frac{\tau_{k}^{-}}{|\tan \varphi|}\right) e^{-\frac{\tau_{k}^{-}}{\sin \varphi \sin \theta}} \\
& +\frac{\sigma}{\pi \sin \varphi \sin \theta} \int_{\tau_{u}^{+}=\tau_{k}^{+}}^{\tau_{0}^{\prime}} T^{4}\left(\tau_{0}^{\prime}-\frac{\tau_{u}^{+}-\tau_{k}^{+}}{|\tan \varphi|}, \tau_{u}^{+}\right) e^{-\frac{\tau_{u}^{+}-\tau_{k}^{+}}{\sin \varphi \sin \theta}} d \tau_{u}^{+}
\end{aligned}
$$

Integrating on the whole surface by applying the variable change $\xi=\varphi-\frac{\pi}{2}$ leads to: 


$$
\begin{aligned}
\frac{\pi}{\sigma} \int_{\varphi=\frac{\pi}{2}}^{\pi-\alpha_{O}^{+}} I_{N}^{+}(\theta, \varphi)|\cos \varphi| d \varphi & \\
= & \int_{\xi=0}^{\alpha_{N}^{+}} T_{R N}^{4}\left(\tau^{\prime}{ }_{0}-\tau_{k}^{-} \tan \xi\right) e^{-\frac{\tau_{k}^{-}}{\cos \xi \sin \theta}} \sin \xi d \xi \\
& +\frac{1}{\sin \theta} \int_{\xi=0}^{\alpha_{N}^{+}} \int_{\tau_{u}^{+}=\hat{y}_{k}}^{\tau^{\prime}{ }_{0}} T^{4}\left[\tau^{\prime}{ }_{0}-\left(\tau_{u}^{+}-\tau_{k}^{+}\right) \tan \xi, \tau_{u}^{+}\right] e^{-\frac{\tau_{u}^{+}-\tau_{k}^{+}}{\cos \xi \sin \theta}} \tan \xi d \xi d \tau_{u}^{+}
\end{aligned}
$$

Integrating over the $\theta$ range by using the special functions $K i_{n}$ writes for the northern contribution:

$$
\begin{aligned}
& \frac{\pi}{\sigma} \int_{\theta=0}^{\frac{\pi}{2}} \int_{\varphi=\frac{\pi}{2}}^{\pi-\alpha_{O}^{+}} I_{N}^{+}(\theta, \varphi)|\cos \varphi| d \varphi \sin ^{2} \theta d \theta \\
& \quad=\int_{\varphi=0}^{\alpha_{N}^{+}} T_{R N}^{4}\left(\tau^{\prime}{ }_{0}-\tau_{k}^{-} \tan \varphi\right) K i_{3}\left(\frac{\tau_{k}^{-}}{\cos \varphi}\right) \sin \varphi d \varphi \\
&+\int_{\varphi=0}^{\alpha_{N}^{+}} \int_{\tau_{u}^{+}=\tau_{k}^{+}}^{\tau^{\prime} 0} T^{4}\left[\tau^{\prime}{ }_{0}-\left(\tau_{u}^{+}-\tau_{k}^{+}\right) \tan \varphi, \tau_{u}^{+}\right] K i_{2}\left(\frac{\tau_{u}^{+}-\tau_{k}^{+}}{\cos \varphi}\right) d \tau_{u}^{+} \tan \varphi d \varphi
\end{aligned}
$$

Performing identical calculations for the other contributions leads to, when noting $\alpha_{O}^{-}=\tan ^{-1}\left(\frac{\hat{y}_{k}}{H}\right)$, $\alpha_{S}^{+}=\frac{\pi}{2}-\alpha_{O}^{-}=\tan ^{-1}\left(\frac{H}{\hat{y}_{k}}\right), \alpha_{\sigma_{S}}^{-}=\frac{\pi}{2}-\alpha_{\sigma_{E}}^{-}=\tan ^{-1}\left(\frac{H-h}{H-h-2 \hat{y}_{k}}\right)$ and $\alpha_{\sigma_{S}}^{+}=\frac{\pi}{2}-\alpha_{O 1}^{+}=\tan ^{-1}\left(\frac{H+h}{H-h-2 \hat{y}_{k}}\right)$ :

- Contribution incoming from the western cavity's surface:

$$
\begin{aligned}
\int_{\theta=0}^{\frac{\pi}{2}} \int_{\varphi=\pi-\alpha_{O}^{+}}^{\pi-\alpha_{\sigma_{E}}^{+}} I_{O}^{+} & (\theta, \varphi)|\cos \varphi| d \varphi \sin ^{2} \theta d \theta+\int_{\theta=0}^{\frac{\pi}{2}} \int_{\varphi=\pi-\alpha_{O}^{+}}^{\pi+\alpha_{O}^{-}} I_{O}^{+}(\theta, \varphi)|\cos \varphi| d \varphi \sin ^{2} \theta d \theta \\
& =\frac{\sigma}{\pi}\left[\int_{\varphi=\alpha_{\sigma_{E}}^{+}}^{\alpha_{O}^{+}} T_{R O}^{4}\left(\tau_{k}^{+}+\tau^{\prime}{ }_{0} \tan \varphi\right) K i_{3}\left(\frac{\tau_{0}^{\prime}{ }_{0}}{\cos \varphi}\right) \cos \varphi d \varphi\right. \\
& +\int_{\varphi=0}^{\alpha_{O 1}^{+}} T_{R O}^{4}\left(\tau_{k}^{+}+\tau_{0}^{\prime} \tan \varphi\right) K i_{3}\left(\frac{\tau_{0}^{\prime}{ }_{0}}{\cos \varphi}\right) \cos \varphi d \varphi \\
& \left.+\int_{\varphi=0}^{\alpha_{O}^{-}} T_{R O}^{4}\left(\tau_{k}^{+}-\tau_{0}^{\prime} \tan \varphi\right) K i_{3}\left(\frac{\tau_{0}^{\prime}}{\cos \varphi}\right) \cos \varphi d \varphi\right] \\
& +\frac{\sigma}{\pi}\left[\int_{\varphi=\alpha_{\sigma_{E}}^{+}}^{\alpha_{O}^{+}} \int_{\tau_{u}^{+}=0}^{\tau^{\prime}{ }_{0}} T^{4}\left(\tau_{u}^{+}, \tau_{k}^{+}+\tau_{u}^{-} \tan \varphi\right) K i_{2}\left(\frac{\tau_{u}^{-}}{\cos \varphi}\right) d \tau_{u}^{+} d \varphi\right. \\
& +\int_{\varphi=0}^{\alpha_{O 1}^{+}} \int_{\tau_{u}^{+}=0}^{\tau_{0}} T^{4}\left(\tau_{u}^{+}, \tau_{k}^{+}+\tau_{u}^{-} \tan \varphi\right) K i_{2}\left(\frac{\tau_{u}^{-}}{\cos \varphi}\right) d \tau_{u}^{+} d \varphi \\
& \left.+\int_{\varphi=0}^{\alpha_{O}^{-}} \int_{\tau_{u}^{+}=0}^{\tau_{0}} T^{4}\left(\tau_{u}^{+}, \tau_{k}^{+}-\tau_{u}^{-} \tan \varphi\right) K i_{2}\left(\frac{\tau_{u}^{-}}{\cos \varphi}\right) d \tau_{u}^{+} d \varphi\right]
\end{aligned}
$$

- Contribution incoming from the southern cavity's surface: 


$$
\begin{aligned}
\frac{\pi}{\sigma} \int_{\theta=0}^{\frac{\pi}{2}} \int_{\varphi=\pi+\alpha_{O}^{-}}^{\frac{3 \pi}{2}} & I_{S}^{+}(\theta, \varphi)|\cos \varphi| d \varphi \sin ^{2} \theta d \theta \\
= & \int_{\varphi=0}^{\alpha_{S}^{+}} T_{R S}^{4}\left(\tau^{\prime}{ }_{0}-\tau_{k}^{+} \tan \varphi\right) K i_{3}\left(\frac{\tau_{k}^{+}}{\cos \varphi}\right) \sin \varphi d \varphi \\
& +\int_{\varphi=0}^{\alpha_{S}^{+}} \int_{\tau_{u}^{+}=0}^{\tau_{k}^{+}} T^{4}\left[\tau_{0}^{\prime}-\left(\tau_{u}^{-}-\tau_{k}^{-}\right) \tan \varphi, \tau_{u}^{+}\right] K i_{2}\left(\frac{\tau_{u}^{-}-\tau_{k}^{-}}{\cos \varphi}\right) d \tau_{u}^{+} \tan \varphi d \varphi
\end{aligned}
$$

- Contribution incoming from the eastern obstacle's surface:

$$
\begin{aligned}
\frac{\pi}{\sigma} \int_{\theta=0}^{\frac{\pi}{2}} \int_{\varphi=\pi-\alpha_{\sigma_{E}}^{+}}^{\pi-\alpha_{\sigma_{E}}} I_{\sigma_{E}}^{+}(\theta, \varphi)|\cos \varphi| d \varphi \sin ^{2} \theta d \theta \\
=\int_{\varphi=\alpha_{\sigma_{E}}^{-}}^{\alpha_{\sigma_{E}}^{+}} T_{R \sigma_{E}}^{4}\left(\tau_{k}^{+}+\tau_{0} \tan \varphi\right) K i_{3}\left(\frac{\tau_{k}^{-}}{\cos \varphi}\right) \cos \varphi d \varphi \\
+\int_{\varphi=\alpha_{\sigma_{E}}^{-}}^{\alpha_{\sigma_{E}}^{+}} \int_{\tau_{u}^{+}=\tau_{0}{ }_{0}}^{\tau \prime_{0}} T^{4}\left(\tau_{u}^{+}, \tau_{k}^{+}+\tau_{u}^{-} \tan \varphi\right) K i_{2}\left(\frac{\tau_{u}^{-}}{\cos \varphi}\right) d \tau_{u}^{+} d \varphi
\end{aligned}
$$

- Contribution incoming from the southern obstacle's surface:

$$
\begin{aligned}
\frac{\pi}{\sigma} \int_{\theta=0}^{\frac{\pi}{2}} \int_{\varphi=\pi-\alpha_{\sigma_{E}}^{-}}^{\pi-\alpha_{O 1}^{+}} I_{\sigma_{S}}^{+}(\theta, \varphi)|\cos \varphi| d \varphi \sin ^{2} \theta d \theta \\
\quad=\int_{\varphi=\alpha_{\sigma_{S}}^{-}}^{\alpha_{\sigma_{S}}^{+}} T_{R \sigma_{S}}^{4}\left[\tau^{\prime}{ }_{0}-\left(\tau_{0}-\tau_{k}^{+}\right) \tan \varphi\right] K i_{3}\left(\frac{\tau_{0}-\tau_{k}^{+}}{\cos \varphi}\right) \sin \varphi d \varphi \\
\quad+\int_{\varphi=\alpha_{\sigma_{S}}^{-}}^{\alpha_{\sigma_{S}}} \int_{\tau_{u}^{+}=\tau_{k}^{+}}^{\tau_{0}} T^{4}\left[\tau_{0}^{\prime}-\left(\tau_{u}^{+}-\tau_{k}^{+}\right) \tan \varphi, \tau_{u}^{+}\right] K i_{2}\left(\frac{\tau_{u}^{+}-\tau_{k}^{+}}{\cos \varphi}\right) d \tau_{u}^{+} \tan \varphi d \varphi
\end{aligned}
$$

whence it comes for the eastern cavity's surface temperature radiosity at the point $M_{k}\left(\hat{y}_{k}\right)$ if $\frac{h}{H} \leq \sqrt{5}-2$ and $\frac{2 h H}{H+h}<\hat{y}_{k} \leq \frac{H-h}{2}$ : 


$$
\begin{aligned}
\frac{\pi\left[T_{R E}^{4}\left(\hat{y}_{k}\right)-\varepsilon_{E} T_{E}^{4}\right]}{2 \rho_{E}} & \\
& =\int_{\varphi=0}^{\alpha_{N}^{+}} T_{R N}^{4}\left(\tau^{\prime}{ }_{0}-\tau_{k}^{-} \tan \varphi\right) K i_{3}\left(\frac{\tau_{k}^{-}}{\cos \varphi}\right) \sin \varphi d \varphi \\
& +\int_{\varphi=\alpha_{\sigma_{E}}^{+}}^{\alpha_{O}^{+}} T_{R O}^{4}\left(\tau_{k}^{+}+\tau_{0}^{\prime} \tan \varphi\right) K i_{3}\left(\frac{\tau_{0}^{\prime}}{\cos \varphi}\right) \cos \varphi d \varphi \\
& +\int_{\varphi=0}^{\alpha_{O 1}^{+}} T_{R O}^{4}\left(\tau_{k}^{+}+\tau_{0}^{\prime} \tan \varphi\right) K i_{3}\left(\frac{\tau_{0}^{\prime}}{\cos \varphi}\right) \cos \varphi d \varphi \\
& +\int_{\varphi=0}^{\alpha_{O}^{-}} T_{R O}^{4}\left(\tau_{k}^{+}-\tau_{0}^{\prime} \tan \varphi\right) K i_{3}\left(\frac{\tau_{0}^{\prime}}{\cos \varphi}\right) \cos \varphi d \varphi \\
& +\int_{\varphi=0}^{\alpha_{S}^{+}} T_{R S}^{4}\left(\tau_{0}^{\prime}-\tau_{k}^{+} \tan \varphi\right) K i_{3}\left(\frac{\tau_{k}^{+}}{\cos \varphi}\right) \sin \varphi d \varphi \\
& +\int_{\varphi=\alpha_{\sigma_{E}}^{-}}^{\alpha_{\sigma_{E}}^{+}} T_{R \sigma_{E}}^{4}\left(\tau_{k}^{+}+\tau_{0} \tan \varphi\right) K i_{3}\left(\frac{\tau_{k}^{-}}{\cos \varphi}\right) \cos \varphi d \varphi \\
& +\int_{\varphi=\alpha_{\sigma_{S}}^{-}}^{\alpha_{\sigma_{S}}^{+}} T_{R \sigma_{S}}^{4}\left[\tau_{0}^{\prime}{ }_{0}-\left(\tau_{0}-\tau_{k}^{+}\right) \tan \varphi\right] K i_{3}\left(\frac{\tau_{0}-\tau_{k}^{+}}{\cos \varphi}\right) \sin \varphi d \varphi+\kappa \aleph_{E}
\end{aligned}
$$

where the internal contribution $\aleph_{E}$ is expressed by, in the general case of an non-isothermal medium:

$$
\begin{aligned}
& \kappa \aleph_{E}=\int_{\varphi=0}^{\alpha_{N}^{+}} \int_{\tau_{u}^{+}=\tau_{k}^{+}}^{\tau \prime} T_{0}^{4}\left[\tau_{0}^{\prime}-\left(\tau_{u}^{+}-\tau_{k}^{+}\right) \tan \varphi, \tau_{u}^{+}\right] K i_{2}\left(\frac{\tau_{u}^{+}-\tau_{k}^{+}}{\cos \varphi}\right) d \tau_{u}^{+} \tan \varphi d \varphi \\
&+\int_{\varphi=\alpha_{\sigma_{E}}^{+}}^{\alpha_{O}^{+}} \int_{\tau_{u}^{+}=0}^{\tau_{0}} T^{4}\left(\tau_{u}^{+}, \tau_{k}^{+}+\tau_{u}^{-} \tan \varphi\right) K i_{2}\left(\frac{\tau_{u}^{-}}{\cos \varphi}\right) d \tau_{u}^{+} d \varphi \\
&+\int_{\varphi=0}^{\alpha_{O 1}^{+}} \int_{\tau_{u}^{+}=0}^{\tau_{0}^{\prime}} T^{4}\left(\tau_{u}^{+}, \tau_{k}^{+}+\tau_{u}^{-} \tan \varphi\right) K i_{2}\left(\frac{\tau_{u}^{-}}{\cos \varphi}\right) d \tau_{u}^{+} d \varphi \\
&+\int_{\varphi=0}^{\alpha_{O}^{-}} \int_{\tau_{u}^{+}=0}^{\tau_{0}^{\prime}} T^{4}\left(\tau_{u}^{+}, \tau_{k}^{+}-\tau_{u}^{-} \tan \varphi\right) K i_{2}\left(\frac{\tau_{u}^{-}}{\cos \varphi}\right) d \tau_{u}^{+} d \varphi \\
&+\int_{\varphi=0}^{\alpha_{S}^{+}} \int_{\tau_{u}^{+}=0}^{\tau_{k}^{+}} T^{4}\left[\tau_{0}^{\prime}-\left(\tau_{u}^{-}-\tau_{k}^{-}\right) \tan \varphi, \tau_{u}^{+}\right] K i_{2}\left(\frac{\tau_{u}^{-}-\tau_{k}^{-}}{\cos \varphi}\right) d \tau_{u}^{+} \tan \varphi d \varphi \\
&+\int_{\varphi=\alpha_{\sigma_{E}}^{-}}^{\alpha_{\sigma_{E}}} \int_{\tau_{u}^{+}=\tau_{0}^{\prime \prime}}^{\tau_{0}} T^{4}\left(\tau_{u}^{+}, \tau_{k}^{+}+\tau_{u}^{-} \tan \varphi\right) K i_{2}\left(\frac{\tau_{u}^{-}}{\cos \varphi}\right) d \tau_{u}^{+} d \varphi \\
&+\int_{\varphi=\alpha_{\sigma_{S}}}^{\alpha_{\sigma_{S}}} \int_{\tau_{u}^{+}=\tau_{k}^{+}}^{\tau_{0}} T^{4}\left[\tau_{0}^{\prime}-\left(\tau_{u}^{+}-\tau_{k}^{+}\right) \tan \varphi, \tau_{u}^{+}\right] K i_{2}\left(\frac{\tau_{u}^{+}-\tau_{k}^{+}}{\cos \varphi}\right) d \tau_{u}^{+} \tan \varphi d \varphi
\end{aligned}
$$

For a constant internal temperature $T$, the $\kappa \aleph_{E}$ function writes:

$$
\begin{aligned}
\frac{\kappa \aleph_{E}}{T^{4}}=\frac{\pi}{2}-B i S_{3} & \left(\tau^{\prime}{ }_{0}, \alpha_{O}^{+}\right)+B i s_{3}\left(\tau^{\prime}{ }_{0}, \alpha_{\sigma_{E}}^{+}\right)-B i s_{3}\left(\tau_{0}^{\prime}, \alpha_{O 1}^{+}\right)-B i s_{3}\left(\tau_{0}^{\prime}, \alpha_{O}^{-}\right)-B i s_{3}\left(\tau_{0}, \alpha_{\sigma_{E}}^{+}\right) \\
& +B i s_{3}\left(\tau_{0}, \alpha_{\sigma_{E}}^{-}\right)-C i s_{3}\left(\tau_{k}^{-}, \alpha_{N}^{+}\right)-\operatorname{Cis}_{3}\left(\tau_{k}^{+}, \alpha_{S}^{+}\right)-C i s_{3}\left(\tau_{0}-\tau_{k}^{+}, \alpha_{\sigma_{S}}^{+}\right) \\
& +C i s_{3}\left(\tau_{0}-\tau_{k}^{+}, \alpha_{\sigma_{S}}^{-}\right)
\end{aligned}
$$


When the whole cavity's northern surface is not completely seen by the observation point $M_{k}\left(\hat{y}_{k}\right)$, which arrives for $\left(\frac{h}{H} \leq \sqrt{5}-2\right.$ and $\left.0 \leq \hat{y}_{k} \leq \frac{2 h H}{H+h}\right)$ or $\left(\frac{h}{H}>\sqrt{5}-2\right.$ and $\left.0 \leq \hat{y}_{k} \leq \frac{H-h}{2}\right)$, the radiosity temperature similarly writes, after defining the angle $\alpha_{N 1}^{+}=\frac{\pi}{2}-\alpha_{\sigma_{E}}^{+}=\tan ^{-1}\left(\frac{H-h}{H+h-2 \hat{y}_{k}}\right)$ :

$$
\begin{aligned}
\frac{\pi\left[T_{R E}^{4}\left(\hat{y}_{k}\right)-\varepsilon_{E} T_{E}^{4}\right]}{2 \rho_{E}} & \\
& =\int_{\varphi=0}^{\alpha_{N 1}^{+}} T_{R N}^{4}\left(\tau_{0}^{\prime}-\tau_{k}^{-} \tan \varphi\right) K i_{3}\left(\frac{\tau_{k}^{-}}{\cos \varphi}\right) \sin \varphi d \varphi \\
& +\int_{\varphi=0}^{\alpha_{O 1}^{+}} T_{R O}^{4}\left(\tau_{k}^{+}+\tau_{0}^{\prime} \tan \varphi\right) K i_{3}\left(\frac{\tau_{0}^{\prime}}{\cos \varphi}\right) \cos \varphi d \varphi \\
& +\int_{\varphi=0}^{\alpha_{O}^{-}} T_{R O}^{4}\left(\tau_{k}^{+}-\tau_{0}^{\prime} \tan \varphi\right) K i_{3}\left(\frac{\tau_{0}^{\prime}}{\cos \varphi}\right) \cos \varphi d \varphi \\
& +\int_{\varphi=0}^{\alpha_{\sigma_{S}}^{+}} T_{R S}^{4}\left(\tau_{0}^{\prime}{ }_{0}-\tau_{k}^{+} \tan \varphi\right) K i_{3}\left(\frac{\tau_{k}^{+}}{\cos \varphi}\right) \sin \varphi d \varphi \\
& +\int_{\varphi=\alpha_{\sigma_{E}}^{-}}^{\alpha_{\sigma_{E}}^{+}} T_{R \sigma_{E}}^{4}\left(\tau_{k}^{+}+\tau_{0} \tan \varphi\right) K i_{3}\left(\frac{\tau_{0}}{\cos \varphi}\right) \cos \varphi d \varphi \\
& +\int_{\varphi=\alpha_{\sigma_{S}}^{-}}^{\alpha_{\sigma_{S}}^{+}} T_{R \sigma_{S}}^{4}\left[\tau_{0}^{\prime}-\left(\tau_{0}-\tau_{k}^{+}\right) \tan \varphi\right] K i_{3}\left(\frac{\tau_{0}-\tau_{k}^{+}}{\cos \varphi}\right) \sin \varphi d \varphi+\kappa \aleph_{E}
\end{aligned}
$$

where the general function $\aleph_{E}$ is given by:

$$
\begin{aligned}
& \kappa \aleph_{E}=\int_{\varphi=0}^{\alpha_{N 1}^{+}} \int_{\tau_{u}^{+}=\tau_{k}^{+}}^{\tau \prime} T^{4}\left[\tau_{0}^{\prime}-\left(\tau_{u}^{+}-\tau_{k}^{+}\right) \tan \varphi, \tau_{u}^{+}\right] K i_{2}\left(\frac{\tau_{u}^{+}-\tau_{k}^{+}}{\cos \varphi}\right) d \tau_{u}^{+} \tan \varphi d \varphi \\
&+\int_{\varphi=0}^{\alpha_{O 1}^{+}} \int_{\tau_{u}^{+}=0}^{\tau_{0}^{\prime}} T^{4}\left(\tau_{u}^{+}, \tau_{k}^{+}+\tau_{u}^{-} \tan \varphi\right) K i_{2}\left(\frac{\tau_{u}^{-}}{\cos \varphi}\right) d \tau_{u}^{+} d \varphi \\
&+\int_{\varphi=0}^{\alpha_{O}^{-}} \int_{\tau_{u}^{+}=0}^{\tau_{0}} T^{4}\left(\tau_{u}^{+}, \tau_{k}^{+}-\tau_{u}^{-} \tan \varphi\right) K i_{2}\left(\frac{\tau_{u}^{-}}{\cos \varphi}\right) d \tau_{u}^{+} d \varphi \\
&+\int_{\varphi=0}^{\alpha_{S}^{+}} \int_{\tau_{u}^{+}=0}^{\tau_{k}^{+}} T^{4}\left[\tau^{\prime}{ }_{0}-\left(\tau_{u}^{-}-\tau_{k}^{-}\right) \tan \varphi, \tau_{u}^{+}\right] K i_{2}\left(\frac{\tau_{u}^{-}-\tau_{k}^{-}}{\cos \varphi}\right) d \tau_{u}^{+} \tan \varphi d \varphi \\
&+\int_{\varphi=\alpha_{\sigma_{E}}}^{\alpha_{\sigma_{E}}^{+}} \int_{\tau_{u}^{+}=\tau_{0}{ }_{0}}^{\tau \prime_{0}} T^{4}\left(\tau_{u}^{+}, \tau_{k}^{+}+\tau_{u}^{-} \tan \varphi\right) K i_{2}\left(\frac{\tau_{u}^{-}}{\cos \varphi}\right) d \tau_{u}^{+} d \varphi \\
&+\int_{\varphi=\alpha_{\sigma_{S}}^{-}}^{\alpha_{\sigma_{S}}} \int_{\tau_{u}^{+}=\tau_{k}^{+}}^{\tau_{0}} T^{4}\left[\tau^{\prime}{ }_{0}-\left(\tau_{u}^{+}-\tau_{k}^{+}\right) \tan \varphi, \tau_{u}^{+}\right] K i_{2}\left(\frac{\tau_{u}^{+}-\tau_{k}^{+}}{\cos \varphi}\right) d \tau_{u}^{+} \tan \varphi d \varphi
\end{aligned}
$$

which for isothermal media reduces to:

$$
\begin{gathered}
\frac{\kappa \aleph_{E}}{T^{4}}=\frac{\pi}{2}-B i s_{3}\left(\tau_{0}^{\prime}, \alpha_{O 1}^{+}\right)-B i s_{3}\left(\tau_{0}^{\prime}, \alpha_{O}^{-}\right)-B i s_{3}\left(\tau_{0}, \alpha_{\sigma_{E}}^{+}\right)+B i s_{3}\left(\tau_{0}, \alpha_{\sigma_{E}}^{-}\right)-C i s_{3}\left(\tau_{k}^{-}, \alpha_{N 1}^{+}\right) \\
-\operatorname{Cis}_{3}\left(\tau_{k}^{+}, \alpha_{S}^{+}\right)-\operatorname{Cis}_{3}\left(\tau_{0}-\tau_{k}^{+}, \alpha_{\sigma_{S}}^{+}\right)+C i s_{3}\left(\tau_{0}-\tau_{k}^{+}, \alpha_{\sigma_{S}}^{-}\right)
\end{gathered}
$$

Let us now examine points for which $\frac{H-h}{2} \leq \hat{y}_{k} \leq \frac{H}{2}$, as illustrated in Fig. 3: 


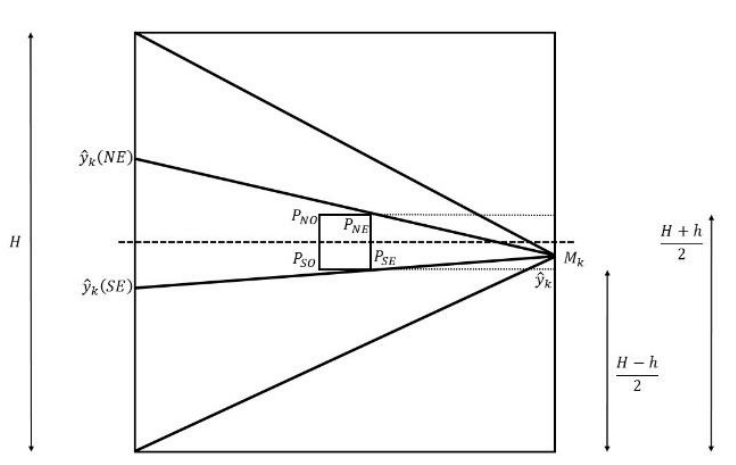

$\frac{h}{H} \leq \sqrt{5}-2$ or $\left(\frac{h}{H}>\sqrt{5}-2\right.$ and $\left.\frac{2 h H}{H+h} \leq \hat{y}_{k} \leq \frac{H}{2}\right)$

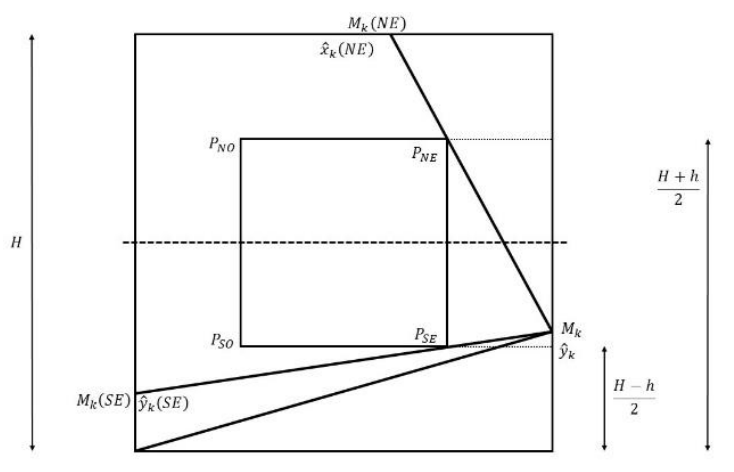

$\frac{h}{H} \geq \frac{1}{3}$ and $\frac{H-h}{2} \leq \hat{y}_{k} \leq \frac{H(H-h)}{H+h}$

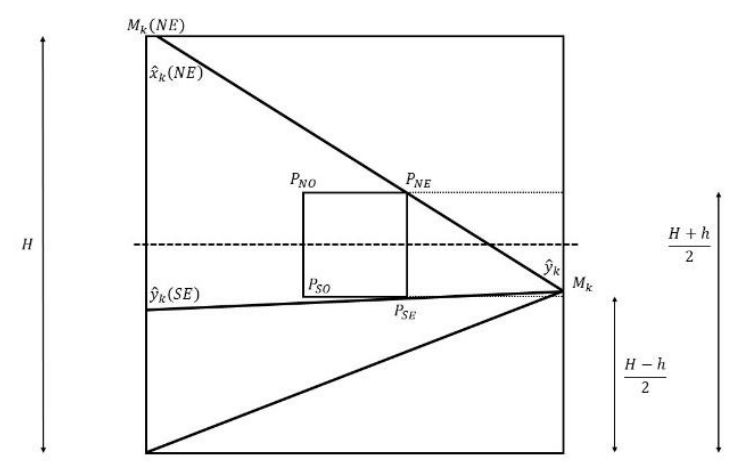

$\frac{h}{H}>\sqrt{5}-2$ and $\frac{H-h}{2} \leq \hat{y}_{k} \leq \frac{2 h H}{H+h}$

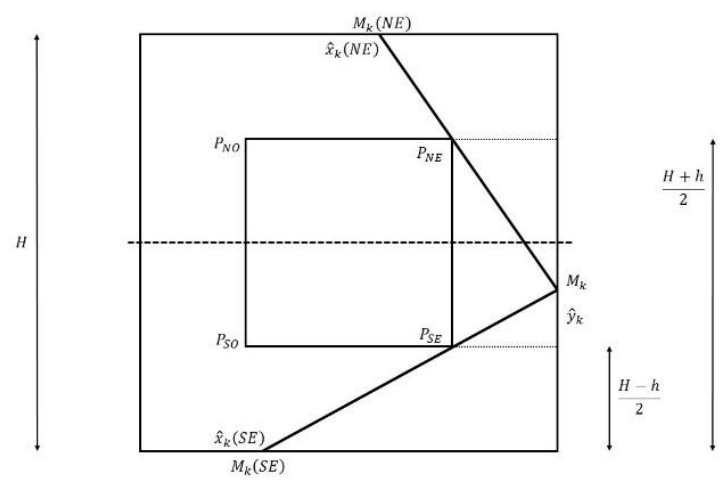

$\frac{h}{H} \geq \frac{1}{3}$ and $\frac{H(H-h)}{H+h} \leq \hat{y}_{k} \leq \frac{H}{2}$

Figure 3: Determination of the eastern cavity's surface radiosity temperature when $\frac{H-h}{2} \leq \hat{y}_{k} \leq \frac{H}{2}$

Note that if $\frac{h}{H} \leq \sqrt{5}-2$, the point $M_{k}(N E)$ is always located on $\Sigma_{O}$, while if $\frac{h}{H}>\sqrt{5}-2$, this point belongs to $\Sigma_{N}$ for $\frac{H-h}{2} \leq \hat{y}_{k} \leq \frac{2 h H}{H+h}$. Furthermore, if $\frac{2 h H}{H+h}<\frac{H}{2}$, or equivalently $\frac{h}{H} \leq \frac{1}{3}$, then the point $M_{k}(N E)$ is on the western surface if $\frac{2 h H}{H+h} \leq \hat{y}_{k} \leq \frac{H}{2}$, while it is always on the northern surface for $\frac{h}{H}>\frac{1}{3}$, which is depicted in Fig. 3

The straight line passing through the two points $M_{k}$ and $P_{S E}$ cuts the cavity's southern surface at the point $M_{k}(S E)$ of abscissa $x=\frac{\hat{y}_{k}(H+h)-H(H-h)}{2 \hat{y}_{k}-H+h}$, whence one deduces from this result that $M_{k}(S E)$ is on $\Sigma_{S}$ if $\hat{y}_{k}>\frac{H(H-h)}{H+h}$ with $\frac{H-h}{2} \leq \hat{y}_{k} \leq \frac{H}{2}$, which implies $\frac{h}{H} \geq \frac{1}{3}$.

The cases $\left(\frac{h}{H}>\sqrt{5}-2\right.$ and $\left.\frac{H-h}{2} \leq \hat{y}_{k} \leq \frac{2 h H}{H+h}\right)$ and $\left(\frac{h}{H} \geq \frac{1}{3}\right.$ and $\left.\frac{H-h}{2} \leq \hat{y}_{k} \leq \frac{H(H-h)}{H+h}\right)$ lead to identical expressions of the radiosity temperature. Finally, the three different cases for points $M_{k}$ located on the partial segment $\frac{H-h}{2} \leq \hat{y}_{k} \leq \frac{H}{2}$ of $\Sigma_{S}$ are summed up hereafter:

- $\left(\frac{h}{H} \leq \sqrt{5}-2\right.$ and $\left.\frac{H-h}{2} \leq \hat{y}_{k} \leq \frac{H}{2}\right)$ or $\left(\frac{h}{H}>\sqrt{5}-2\right.$ and $\left.\frac{2 h H}{H+h} \leq \hat{y}_{k} \leq \frac{H}{2}\right)$, 


$$
\begin{aligned}
\frac{\pi\left[T_{R E}^{4}\left(\hat{y}_{k}\right)-\varepsilon_{E} T_{E}^{4}\right]}{2 \rho_{E}} & \\
& =\int_{\varphi=0}^{\alpha_{N}^{+}} T_{R N}^{4}\left(\tau^{\prime}{ }_{0}-\tau_{k}^{-} \tan \varphi\right) K i_{3}\left(\frac{\tau_{k}^{-}}{\cos \varphi}\right) \sin \varphi d \varphi \\
& +\int_{\varphi=\alpha_{\sigma_{E}}^{+}}^{\alpha_{O}^{+}} T_{R O}^{4}\left(\tau_{k}^{+}+\tau_{0}^{\prime} \tan \varphi\right) K i_{3}\left(\frac{\tau_{0}^{\prime}}{\cos \varphi}\right) \cos \varphi d \varphi \\
& +\int_{\varphi=\alpha_{\sigma_{E}}^{-}}^{\alpha_{O}^{-}} T_{R O}^{4}\left(\tau_{k}^{+}-\tau_{0}^{\prime} \tan \varphi\right) K i_{3}\left(\frac{\tau_{0}^{\prime}}{\cos \varphi}\right) \cos \varphi d \varphi \\
& +\int_{\varphi=0}^{\alpha_{S}^{+}} T_{R S}^{4}\left(\tau_{0}^{\prime}-\tau_{k}^{+} \tan \varphi\right) K i_{3}\left(\frac{\tau_{k}^{+}}{\cos \varphi}\right) \sin \varphi d \varphi \\
& +\int_{\varphi=0}^{\alpha_{\sigma_{E}}^{+}} T_{R \sigma_{E}}^{4}\left(\tau_{k}^{+}+\tau_{0} \tan \varphi\right) K i_{3}\left(\frac{\tau_{0}}{\cos \varphi}\right) \cos \varphi d \varphi \\
& +\int_{\varphi=0}^{\alpha_{\sigma_{E}}} T_{R \sigma_{E}}^{4}\left(\tau_{k}^{+}-\tau_{0} \tan \varphi\right) K i_{3}\left(\frac{\tau_{0}}{\cos \varphi}\right) \cos \varphi d \varphi+\kappa \aleph_{E}
\end{aligned}
$$

where this time the angle $\alpha_{\sigma_{E}}^{-}$is $\alpha_{\sigma_{E}}^{-}=\tan ^{-1}\left(\frac{2 \hat{y}_{k}-H+h}{H-h}\right)$ and the function $\aleph_{E}$ writes:

$$
\begin{aligned}
& \kappa \aleph_{E}=\int_{\varphi=0}^{\alpha_{N}^{+}} \int_{\tau_{u}^{+}=\tau_{k}^{+}}^{\tau^{\prime}} T^{4}\left[\tau_{0}^{\prime}-\left(\tau_{u}^{+}-\tau_{k}^{+}\right) \tan \varphi, \tau_{u}^{+}\right] K i_{2}\left(\frac{\tau_{u}^{+}-\tau_{k}^{+}}{\cos \varphi}\right) d \tau_{u}^{+} \tan \varphi d \varphi \\
&+\int_{\varphi=\alpha_{\sigma_{E}}^{+}}^{\alpha_{O}^{+}} \int_{\tau_{u}^{+}=0}^{\tau \prime_{0}} T^{4}\left(\tau_{u}^{+}, \tau_{k}^{+}+\tau_{u}^{-} \tan \varphi\right) K i_{2}\left(\frac{\tau_{u}^{-}}{\cos \varphi}\right) d \tau_{u}^{+} d \varphi \\
&+\int_{\varphi=\alpha_{\sigma_{E}}^{-}}^{\alpha_{O}^{-}} \int_{\tau_{u}^{+}=0}^{\tau_{0}^{\prime}} T^{4}\left(\tau_{u}^{+}, \tau_{k}^{+}-\tau_{u}^{-} \tan \varphi\right) K i_{2}\left(\frac{\tau_{u}^{-}}{\cos \varphi}\right) d \tau_{u}^{+} d \varphi \\
&+\int_{\varphi=0}^{\alpha_{S}^{+}} \int_{\tau_{u}^{+}=0}^{\tau_{k}^{+}} T^{4}\left[\tau_{0}^{\prime}-\left(\tau_{u}^{-}-\tau_{k}^{-}\right) \tan \varphi, \tau_{u}^{+}\right] K i_{2}\left(\frac{\tau_{u}^{-}-\tau_{k}^{-}}{\cos \varphi}\right) d \tau_{u}^{+} \tan \varphi d \varphi \\
&+\int_{\varphi=0}^{\alpha_{\sigma_{E}}^{+}} \int_{\tau_{u}^{+}=\tau_{0}^{\prime \prime}{ }_{0}}^{\tau \prime_{0}} T^{4}\left(\tau_{u}^{+}, \tau_{k}^{+}+\tau_{u}^{-} \tan \varphi\right) K i_{2}\left(\frac{\tau_{u}^{-}}{\cos \varphi}\right) d \tau_{u}^{+} d \varphi \\
&+\int_{\varphi=0}^{\alpha_{\sigma_{E}}^{-}} \int_{\tau_{u}^{+}=\tau_{0}^{\prime \prime}{ }_{0}}^{\tau_{0}} T^{4}\left(\tau_{u}^{+}, \tau_{k}^{+}-\tau_{u}^{-} \tan \varphi\right) K i_{2}\left(\frac{\tau_{u}^{-}}{\cos \varphi}\right) d \tau_{u}^{+} d \varphi
\end{aligned}
$$

which for isothermal media leads to:

$$
\begin{gathered}
\frac{\kappa \aleph_{E}}{T^{4}}=\frac{\pi}{2}-B i s_{3}\left(\tau_{0}^{\prime}, \alpha_{O}^{+}\right)+B i s_{3}\left(\tau_{0}^{\prime}, \alpha_{\sigma_{E}}^{+}\right)-B i s_{3}\left(\tau_{0}^{\prime}, \alpha_{O}^{-}\right)+B i s_{3}\left(\tau_{0}^{\prime}, \alpha_{\sigma_{E}}^{-}\right)-B i s_{3}\left(\tau_{0}, \alpha_{\sigma_{E}}^{+}\right) \\
-B i s_{3}\left(\tau_{0}, \alpha_{\sigma_{E}}^{-}\right)-C i s_{3}\left(\tau_{k}^{-}, \alpha_{N}^{+}\right)-C i s_{3}\left(\tau_{k}^{+}, \alpha_{S}^{+}\right)
\end{gathered}
$$

- $\left(\sqrt{5}-2 \leq \frac{h}{H}<\frac{1}{3}\right.$ and $\left.\frac{H-h}{2} \leq \hat{y}_{k} \leq \frac{2 h H}{H+h}\right)$ or $\left(\frac{h}{H} \geq \frac{1}{3}\right.$ and $\left.\frac{H-h}{2} \leq \hat{y}_{k} \leq \frac{H(H-h)}{H+h}\right)$, 


$$
\begin{aligned}
\frac{\pi\left[T_{R E}^{4}\left(\hat{y}_{k}\right)-\varepsilon_{E} T_{E}^{4}\right]}{2 \rho_{E}} & \\
& =\int_{\varphi=0}^{\alpha_{N 1}^{+}} T_{R N}^{4}\left(\tau^{\prime}{ }_{0}-\tau_{k}^{-} \tan \varphi\right) K i_{3}\left(\frac{\tau_{k}^{-}}{\cos \varphi}\right) \sin \varphi d \varphi \\
& +\int_{\varphi=\alpha_{\sigma_{E}}^{-}}^{\alpha_{O}^{-}} T_{R O}^{4}\left(\tau_{k}^{+}-\tau^{\prime}{ }_{0} \tan \varphi\right) K i_{3}\left(\frac{\tau_{0}^{\prime}}{\cos \varphi}\right) \cos \varphi d \varphi \\
& +\int_{\varphi=0}^{\alpha_{S}^{+}} T_{R S}^{4}\left(\tau_{0}^{\prime}-\tau_{k}^{+} \tan \varphi\right) K i_{3}\left(\frac{\tau_{k}^{+}}{\cos \varphi}\right) \sin \varphi d \varphi \\
& +\int_{\varphi=0}^{\alpha_{\sigma_{E}}^{+}} T_{R \sigma_{E}}^{4}\left(\tau_{k}^{+}+\tau_{0} \tan \varphi\right) K i_{3}\left(\frac{\tau_{0}}{\cos \varphi}\right) \cos \varphi d \varphi \\
& +\int_{\varphi=0}^{\alpha_{\sigma_{E}}} T_{R \sigma_{E}}^{4}\left(\tau_{k}^{+}-\tau_{0} \tan \varphi\right) K i_{3}\left(\frac{\tau_{0}}{\cos \varphi}\right) \cos \varphi d \varphi+\kappa \aleph_{E}
\end{aligned}
$$

where similarly the general internal contribution writes:

$$
\begin{aligned}
& \kappa \aleph_{E}=\int_{\varphi=0}^{\alpha_{N 1}^{+}} \int_{\tau_{u}^{+}=\tau_{k}^{+}}^{\tau^{\prime} 0} T^{4}\left[\tau^{\prime}{ }_{0}-\left(\tau_{u}^{+}-\tau_{k}^{+}\right) \tan \varphi, \tau_{u}^{+}\right] K i_{2}\left(\frac{\tau_{u}^{+}-\tau_{k}^{+}}{\cos \varphi}\right) d \tau_{u}^{+} \tan \varphi d \varphi \\
&+\int_{\varphi=\alpha_{\sigma_{E}}^{-}}^{\alpha_{O}^{-}} \int_{\tau_{u}^{+}=0}^{\tau_{0}^{\prime}} T^{4}\left(\tau_{u}^{+}, \tau_{k}^{+}-\tau_{u}^{-} \tan \varphi\right) K i_{2}\left(\frac{\tau_{u}^{-}}{\cos \varphi}\right) d \tau_{u}^{+} d \varphi \\
&+\int_{\varphi=0}^{\alpha_{S}^{+}} \int_{\tau_{u}^{+}=0}^{\tau_{k}^{+}} T^{4}\left[\tau_{0}^{\prime}-\left(\tau_{u}^{-}-\tau_{k}^{-}\right) \tan \varphi, \tau_{u}^{+}\right] K i_{2}\left(\frac{\tau_{u}^{-}-\tau_{k}^{-}}{\cos \varphi}\right) d \tau_{u}^{+} \tan \varphi d \varphi \\
&+\int_{\varphi=0}^{\alpha_{\sigma_{E}}^{+}} \int_{\tau_{u}^{+}=\tau_{0}^{\prime \prime}{ }_{0}}^{\tau_{0}} T^{4}\left(\tau_{u}^{+}, \tau_{k}^{+}+\tau_{u}^{-} \tan \varphi\right) K i_{2}\left(\frac{\tau_{u}^{-}}{\cos \varphi}\right) d \tau_{u}^{+} d \varphi \\
&+\int_{\varphi=0}^{\alpha_{\sigma_{E}}^{-}} \int_{\tau_{u}^{+}=\tau_{0}^{\prime \prime}{ }_{0}}^{\tau_{0}} T^{4}\left(\tau_{u}^{+}, \tau_{k}^{+}-\tau_{u}^{-} \tan \varphi\right) K i_{2}\left(\frac{\tau_{u}^{-}}{\cos \varphi}\right) d \tau_{u}^{+} d \varphi
\end{aligned}
$$

which for isothermal media simplifies into:

$$
\begin{gathered}
\frac{\kappa \aleph_{E}}{T^{4}}=\frac{\pi}{2}-B i s_{3}\left(\tau_{0}^{\prime}, \alpha_{O}^{-}\right)+B i s_{3}\left(\tau^{\prime}{ }_{0}, \alpha_{\sigma_{E}}^{-}\right)-B i s_{3}\left(\tau_{0}, \alpha_{\sigma_{E}}^{+}\right)-B i s_{3}\left(\tau_{0}, \alpha_{\sigma_{E}}^{-}\right)-C i s_{3}\left(\tau_{k}^{-}, \alpha_{N 1}^{+}\right) \\
-C i s_{3}\left(\tau_{k}^{+}, \alpha_{S}^{+}\right)
\end{gathered}
$$

and finally, when introducing the angle $\alpha_{S 1}^{+}=\frac{\pi}{2}-\alpha_{\sigma_{E}}^{-}=\tan ^{-1}\left(\frac{H-h}{2 \hat{y}_{k}-H+h}\right)$

- $\frac{h}{H} \geq \frac{1}{3}$ and $\frac{H(H-h)}{H+h} \leq \hat{y}_{k} \leq \frac{H}{2}$, 


$$
\begin{aligned}
\frac{\pi\left[T_{R E}^{4}\left(\hat{y}_{k}\right)-\varepsilon_{E} T_{E}^{4}\right]}{2 \rho_{E}} & \\
& =\int_{\varphi=0}^{\alpha_{N 1}^{+}} T_{R N}^{4}\left(\tau^{\prime}{ }_{0}-\tau_{k}^{-} \tan \varphi\right) K i_{3}\left(\frac{\tau_{k}^{-}}{\cos \varphi}\right) \sin \varphi d \varphi \\
& +\int_{\varphi=0}^{\alpha_{S 1}^{+}} T_{R S}^{4}\left(\tau^{\prime}{ }_{0}-\tau_{k}^{+} \tan \varphi\right) K i_{3}\left(\frac{\tau_{k}^{+}}{\cos \varphi}\right) \sin \varphi d \varphi \\
& +\int_{\varphi=0}^{\alpha_{\sigma_{E}}^{+}} T_{R \sigma_{E}}^{4}\left(\tau_{k}^{+}+\tau_{0} \tan \varphi\right) K i_{3}\left(\frac{\tau_{0}}{\cos \varphi}\right) \cos \varphi d \varphi \\
& +\int_{\varphi=0}^{\alpha_{\sigma_{E}}^{-}} T_{R \sigma_{E}}^{4}\left(\tau_{k}^{+}-\tau_{0} \tan \varphi\right) K i_{3}\left(\frac{\tau_{0}}{\cos \varphi}\right) \cos \varphi d \varphi+\kappa \aleph_{E}
\end{aligned}
$$

where the global internal contribution writes:

$$
\begin{aligned}
& \kappa \aleph_{E}=\int_{\varphi=0}^{\alpha_{N 1}^{+}} \int_{\tau_{u}^{+}=\tau_{k}^{+}}^{\tau \prime} T^{4}\left[\tau_{0}^{\prime}-\left(\tau_{u}^{+}-\tau_{k}^{+}\right) \tan \varphi, \tau_{u}^{+}\right] K i_{2}\left(\frac{\tau_{u}^{+}-\tau_{k}^{+}}{\cos \varphi}\right) d \tau_{u}^{+} \tan \varphi d \varphi \\
&+\int_{\varphi=0}^{\alpha_{S 1}^{+}} \int_{\tau_{u}^{+}=0}^{\tau_{k}^{+}} T^{4}\left[\tau^{\prime}{ }_{0}-\left(\tau_{u}^{-}-\tau_{k}^{-}\right) \tan \varphi, \tau_{u}^{+}\right] K i_{2}\left(\frac{\tau_{u}^{-}-\tau_{k}^{-}}{\cos \varphi}\right) d \tau_{u}^{+} \tan \varphi d \varphi \\
&+\int_{\varphi=0}^{\alpha_{\sigma_{E}}^{+}} \int_{\tau_{u}^{+}=\tau_{0}^{\prime \prime}{ }_{0}}^{\tau_{0}} T^{4}\left(\tau_{u}^{+}, \tau_{k}^{+}+\tau_{u}^{-} \tan \varphi\right) K i_{2}\left(\frac{\tau_{u}^{-}}{\cos \varphi}\right) d \tau_{u}^{+} d \varphi \\
&+\int_{\varphi=0}^{\alpha_{\sigma_{E}}} \int_{\tau_{u}^{+}=\tau_{0}^{\prime \prime}{ }_{0}}^{\tau \prime_{0}} T^{4}\left(\tau_{u}^{+}, \tau_{k}^{+}-\tau_{u}^{-} \tan \varphi\right) K i_{2}\left(\frac{\tau_{u}^{-}}{\cos \varphi}\right) d \tau_{u}^{+} d \varphi
\end{aligned}
$$

In this latter case, the isothermal medium contribution $\kappa \aleph_{E}$ reduces to:

$$
\frac{\kappa \aleph_{E}}{T^{4}}=\frac{\pi}{2}-B i s_{3}\left(\tau_{0}, \alpha_{\sigma_{E}}^{+}\right)-B i s_{3}\left(\tau_{0}, \alpha_{\sigma_{E}}^{-}\right)-C i s_{3}\left(\tau_{k}^{-}, \alpha_{N 1}^{+}\right)-C i s_{3}\left(\tau_{k}^{+}, \alpha_{S 1}^{+}\right)
$$

For points $M_{k}$ on the upper part of the eastern surface with $\hat{y}_{k} \geq \frac{H}{2}$, one performs the formal variable change $y \leftrightarrow H-y$ for the horizontal surfaces arguments and $T_{R N}^{4} \leftrightarrow T_{R S}^{4}$, whence if $\frac{H}{2} \leq \hat{y}_{k} \leq \frac{H+h}{2}$, one deduces from what precedes the different expressions of the cavity's eastern radiosity temperature, reported in the Annex 2.

The western obstacle's surface radiosity temperature is deduced from the previous results by applying the formal substitution $\hat{x} \leftrightarrow H-\hat{x}$ relatively to $\Sigma_{E}$ in the temperatures integrals and adding the same change in the argument of the surfaces northern and southern temperatures. Similarly the radiosity temperature for $\Sigma_{N}$ is obtained with the substitution $\hat{y} \leftrightarrow \hat{x}$ and for $\Sigma_{S}$ one applies $\hat{y} \leftrightarrow H-\hat{y}$ relatively to $\Sigma_{N}$.

The general equations for the radiosity temperatures have to be now discretized, which shall be described in the next section.

\section{III - DISCRETISATION OF THE RADIOSITY TEMPERATURES EQUATIONS}

\section{III-1. The obstacle's radiosity temperatures}

The integrals containing the radiosity temperatures are discretized in the following way, firstly exemplified on the obstacle's surfaces. For the eastern surface, the observation points are located on the segment $\frac{H-h}{2} \leq \hat{y}_{k} \leq \frac{H+h}{2}$ for $k \in\{M, \ldots, M+2 P-6\}$ with 3 punctual cells. 
For any point $M_{k}$ on $\sigma_{E}$, the generic point of ordinate $\tilde{y}=\hat{y}_{k}+\frac{H-h}{2} \tan \varphi$ covers the whole upper surface $\Sigma_{E}$ above $M_{k}$ of ordinate $\hat{y}_{k}$ for a propagation angle $\varphi \in\left[0, \xi_{E}^{+}\right]$.

For a non-punctual cell $(k \neq\{M, M+P-1, M+2 P-2\})$, the contribution of $\Sigma_{E}$ above $M_{k}$ can be discretized as:

$$
\begin{aligned}
\int_{\varphi=0}^{\xi_{E}^{+}} T_{R E}^{4}\left(\tau_{k}^{+}+\right. & \left.\tau_{0} \tan \varphi\right) K i_{3}\left(\frac{\tau_{0}}{\cos \varphi}\right) \cos \varphi d \varphi \\
& =T_{R E, k}^{4} \int_{\varphi=0}^{\varphi_{k}^{+}} K i_{3}\left(\frac{\tau_{0}}{\cos \varphi}\right) \cos \varphi d \varphi+\sum_{m=k+1}^{2(M+P-2)} T_{R E, m}^{4} \int_{\varphi=\varphi_{m}^{-}}^{\varphi_{m}^{+}} K i_{3}\left(\frac{\tau_{0}}{\cos \varphi}\right) \cos \varphi d \varphi
\end{aligned}
$$

where the two angles $\varphi_{m}^{-}$and $\varphi_{m}^{+}$limiting the whole cell labelled by its index $m$ are always defined by $\hat{y}_{m}-\frac{\Delta \hat{y}}{2}=\hat{y}_{k}+\frac{H-h}{2} \tan \varphi_{m}^{-}$and $\hat{y}_{m}+\frac{\Delta \hat{y}}{2}=\hat{y}_{k}+\frac{H-h}{2} \tan \varphi_{m}^{+}$, or equivalently $\varphi_{m}^{-}=\tan ^{-1}\left[\frac{2\left(\hat{y}_{m}-\hat{y}_{k}\right)-\Delta \hat{y}}{H-h}\right]$ and $\varphi_{m}^{+}=\tan ^{-1}\left[\frac{2\left(\hat{y}_{m}-\hat{y}_{k}\right)+\Delta \hat{y}}{H-h}\right]$.

In the formal sum of Eq. (40), the indices of the punctual cells $\{M, M+P-1, M+2 P-2\}$ are omitted because these cells are of zero depth. Using the $\mathrm{Bis}_{3}$ function implies:

$$
\begin{aligned}
\int_{\varphi=0}^{\xi_{E}^{+}} T_{R E}^{4}\left(\tau_{k}^{+}+\right. & \left.\tau_{0} \tan \varphi\right) K i_{3}\left(\frac{\tau_{0}}{\cos \varphi}\right) \cos \varphi d \varphi \\
& =T_{R E, k}^{4} B i s_{3}\left(\tau_{0}, \varphi_{k}^{+}\right)+\sum_{m=k+1}^{2(M+P-2)} T_{R E, m}^{4}\left[B i s_{3}\left(\tau_{0}, \varphi_{m}^{+}\right)-B i s_{3}\left(\tau_{0}, \varphi_{m}^{-}\right)\right]
\end{aligned}
$$

Similarly, the point of ordinate $\tilde{y}=\hat{y}_{k}-\frac{H-h}{2} \tan \varphi$ covers the whole upper surface $\Sigma_{E}$ below $M_{k}$ for a propagation angle $\varphi \in\left[0, \tan ^{-1}\left(\frac{2 \hat{y}_{k}}{H-h}\right)\right]$, and for the same non-punctual cell of index $k$, the contribution of $\Sigma_{E}$ below $M_{k}$ can be formally discretized as:

$$
\begin{aligned}
\int_{\varphi=0}^{\xi_{E}^{-}} T_{R E}^{4}\left(\tau_{k}^{+}-\right. & \left.\tau_{0} \tan \varphi\right) K i_{3}\left(\frac{\tau_{0}}{\cos \varphi}\right) \cos \varphi d \varphi \\
= & \sum_{m=2}^{k-1} T_{R E, m}^{4} \int_{\varphi=\widetilde{\varphi}_{m}^{+}}^{\widetilde{\varphi}_{m}^{-}} K i_{3}\left(\frac{\tau_{0}}{\cos \varphi}\right) \cos \varphi d \varphi+T_{R E, k}^{4} \int_{\varphi=0}^{\widetilde{\varphi}_{k}^{-}} K i_{3}\left(\frac{\tau_{0}}{\cos \varphi}\right) \cos \varphi d \varphi
\end{aligned}
$$

where the two boundary angles $\tilde{\varphi}_{m}^{-}$at the lower limit of the cell labelled $m$ and $\tilde{\varphi}_{m}^{+}$at the lower limit of the same cell are defined by $\hat{y}_{m}-\frac{\Delta \hat{y}}{2}=\hat{y}_{k}-\frac{H-h}{2} \tan \tilde{\varphi}_{m}^{-}$and $\hat{y}_{m}+\frac{\Delta \hat{y}}{2}=\hat{y}_{k}-\frac{H-h}{2} \tan \tilde{\varphi}_{m}^{+}$. In the formal sum (42), the indices of the punctual cells $\{M, M+P-1, M+2 P-2\}$ are still omitted. The previous expression can be rewritten with the $\mathrm{Bis}_{3}$ function under the form:

$$
\begin{aligned}
\int_{\varphi=0}^{\xi_{E}^{-}} T_{R E}^{4}\left(\tau_{k}^{+}-\right. & \left.\tau_{0} \tan \varphi\right) K i_{3}\left(\frac{\tau_{0}}{\cos \varphi}\right) \cos \varphi d \varphi \\
= & \sum_{m=2}^{k-1} T_{R E, m}^{4}\left[B i s_{3}\left(\tau_{0}, \tilde{\varphi}_{m}^{-}\right)-B i s_{3}\left(\tau_{0}, \tilde{\varphi}_{m}^{+}\right)\right]+T_{R E, k}^{4} B i s_{3}\left(\tau_{0}, \tilde{\varphi}_{k}^{-}\right)
\end{aligned}
$$


whence the total contribution of $\Sigma_{E}$ to $\sigma_{E}$ 's radiosity temperature is given by:

$$
\begin{aligned}
\int_{\varphi=0}^{\xi_{E}^{+}} T_{R E}^{4}\left(\tau_{k}^{+}+\right. & \left.\tau_{0} \tan \varphi\right) K i_{3}\left(\frac{\tau_{0}}{\cos \varphi}\right) \cos \varphi d \varphi+\int_{\varphi=0}^{\xi_{E}^{-}} T_{R E}^{4}\left(\tau_{k}^{+}-\tau_{0} \tan \varphi\right) K i_{3}\left(\frac{\tau_{0}}{\cos \varphi}\right) \cos \varphi d \varphi \\
& =\sum_{m=2}^{k-1} T_{R E, m}^{4}\left[B i s_{3}\left(\tau_{0}, \tilde{\varphi}_{m}^{-}\right)-B i s_{3}\left(\tau_{0}, \tilde{\varphi}_{m}^{+}\right)\right]+2 T_{R E, k}^{4} B i s_{3}\left(\tau_{0}, \varphi_{k}^{+}\right) \\
& +\sum_{m=k+1}^{2(M+P-2)} T_{R E, m}^{4}\left[B i s_{3}\left(\tau_{0}, \varphi_{m}^{+}\right)-B i s_{3}\left(\tau_{0}, \varphi_{m}^{-}\right)\right]
\end{aligned}
$$

which can formally be summed up under the generic form:

$$
\begin{aligned}
\int_{\varphi=0}^{\xi_{E}^{+}} T_{R E}^{4}\left(\tau_{k}^{+}+\right. & \left.\tau_{0} \tan \varphi\right) K i_{3}\left(\frac{\tau_{0}}{\cos \varphi}\right) \cos \varphi d \varphi+\int_{\varphi=0}^{\xi_{E}} T_{R E}^{4}\left(\tau_{k}^{+}-\tau_{0} \tan \varphi\right) K i_{3}\left(\frac{\tau_{0}}{\cos \varphi}\right) \cos \varphi d \varphi \\
& =\sum_{m=2}^{2(M+P-2)} C_{k, m}^{E, \sigma_{E}} T_{R E, m}^{4}
\end{aligned}
$$

Note that for the three punctual cells $k=\{M, M+P-1, M+2 P-2\}$ the contribution of the whole surface $\Sigma_{E}$ still writes under the expression given by Eq. (45), with the changes $C_{k, k}^{E, \sigma_{E}}=0$ and $C_{k, k-1}^{E, \sigma_{E}}=C_{k, k+1}^{E, \sigma_{E}}=B i s_{3}\left[\tau_{0}, \tan ^{-1}\left(\frac{2 \Delta \hat{y}}{H-h}\right)\right]$

For the contribution originating from the cavity's northern surface, one has for any point $M_{k}\left(\hat{y}_{k}\right)$ on $\sigma_{E}$ :

$$
\begin{aligned}
C_{N}(k)=\int_{\varphi=0}^{\xi_{N}^{+}} T_{R N}^{4}\left(\tau^{\prime \prime}{ }_{0}+\tau_{k}^{-} \tan \varphi\right) K i_{3}\left(\frac{\tau_{k}^{-}}{\cos \varphi}\right) \sin \varphi d \varphi \\
=T_{R N, M+2 P-1}^{4} \int_{\varphi=0}^{\varphi_{M+2 P-1}^{+}} K i_{3}\left(\frac{\tau_{k}^{-}}{\cos \varphi}\right) \sin \varphi d \varphi \\
+\sum_{m=M+2 P}^{2(M+P-2)} T_{R N, m}^{4} \int_{\varphi=\varphi_{m}^{-}}^{\varphi_{m}^{+}} K i_{3}\left(\frac{\tau_{k}^{-}}{\cos \varphi}\right) \sin \varphi d \varphi
\end{aligned}
$$

where this time the two angles $\hat{\varphi}_{m}^{-}$and $\hat{\varphi}_{m}^{+}$are defined by $\frac{H+h}{2}+\left(H-\hat{y}_{k}\right) \tan \hat{\varphi}_{m}^{-}=\hat{x}_{m}-\frac{\Delta \hat{x}}{2}$ and $\frac{H+h}{2}+\left(H-\hat{y}_{k}\right) \tan \hat{\varphi}_{m}^{+}=\hat{x}_{m}+\frac{\Delta \hat{x}}{2}$, with $\hat{x}_{m}>\frac{H+h}{2}$, from which Eq. (44) rewrites:

$$
C_{N}(k)=T_{R N, M+2 P-1}^{4} \operatorname{Cis}_{3}\left(\tau_{k}^{-}, \hat{\varphi}_{M+2 P-1}^{+}\right)+\sum_{m=M+2 P}^{2(M+P-2)} T_{R N, m}^{4}\left[C i s_{3}\left(\tau_{k}^{-}, \hat{\varphi}_{m}^{+}\right)-\operatorname{Cis}_{3}\left(\tau_{k}^{-}, \hat{\varphi}_{m}^{-}\right)\right]
$$

or in a more synthetic form, introducing the coefficients $C_{k, m}^{N, \sigma_{E}}, C_{N}(k)=\sum_{m=M+2 P-1}^{2(M+P-2)} C_{k, m}^{N, \sigma_{E}} T_{R N, m}^{4}$. The contribution of the cavity's southern surface to the $\sigma_{E}$ 's radiosity temperature expresses exactly in the same way, simply by using the formal substitution $H-\hat{y}_{k} \leftrightarrow \hat{y}_{k}$ leading to: 


$$
\begin{aligned}
C_{S}(k)=\int_{\varphi=0}^{\xi_{S}^{+}} & T_{R S}^{4}\left(\tau^{\prime \prime}{ }_{0}+\tau_{k}^{+} \tan \varphi\right) K i_{3}\left(\frac{\tau_{k}^{+}}{\cos \varphi}\right) \sin \varphi d \varphi \\
& =T_{R S, M+2 P-1}^{4} \operatorname{Cis}_{3}\left(\tau_{k}^{+}, \tilde{\varphi}_{M+2 P-1}^{+}\right)+\sum_{m=M+2 P}^{2(M+P-2)} T_{R S, m}^{4}\left[\operatorname{Cis}_{3}\left(\tau_{k}^{+}, \tilde{\varphi}_{m}^{+}\right)-\operatorname{Cis}_{3}\left(\tau_{k}^{+}, \tilde{\varphi}_{m}^{-}\right)\right]
\end{aligned}
$$

where the angles $\tilde{\varphi}_{m}^{-}$and $\tilde{\varphi}_{m}^{+}$for the southern cavity surface are defined by $\frac{H+h}{2}+\hat{y}_{k} \tan \tilde{\varphi}_{m}^{-}=\hat{x}_{m}-\frac{\Delta \hat{x}}{2}$ and $\frac{H+h}{2}+\hat{y}_{k} \tan \tilde{\varphi}_{m}^{+}=\hat{x}_{m}+\frac{\Delta \hat{x}}{2}$, or in a more synthetic form, introducing the coefficients $C_{k, m}^{S, \sigma_{E}}$, as $C_{S}(k)=\sum_{m=M+2 P-1}^{2(M+P-2)} C_{k, m}^{S, \sigma_{E}} T_{R S, m}^{4}$.

Then the obstacle's eastern surface radiosity temperature writes in its discrete form:

$$
\frac{\pi\left[T_{R \sigma_{E}}^{4}\left(\hat{y}_{k}\right)-\varepsilon_{\sigma_{E}} T_{\sigma_{E}}^{4}\right]}{2 \rho_{\sigma_{E}}}=\sum_{m=2}^{2(M+P-2)}\left(C_{k, m}^{E, \sigma_{E}} T_{R E, m}^{4}+C_{k, m}^{N, \sigma_{E}} T_{R N, m}^{4}+C_{k, m}^{S, \sigma_{E}} T_{R S, m}^{4}\right)+\kappa \aleph_{\sigma_{E}}\left(\hat{y}_{k}\right)
$$

In the global expression of Eq. (49), some coefficients can be equal to 0 .

The three other radiosity temperatures of the obstacle are obtained equivalently as what precedes.

The discrete coefficients $C_{k, m}^{O, \sigma_{O}}$ in the partial contribution $C_{k, m}^{O, \sigma_{O}} T_{R O, m}^{4}$ of the radiosity temperature of $\sigma_{O}$ have exactly the same expressions of the coefficients $C_{k, m}^{E, \sigma_{E}}$ for a given index $k$. Furthermore, the northern contribution of the cavity to the obstacle's western surface writes:

$$
\begin{aligned}
\int_{\varphi=0}^{\xi_{N}^{+}} T_{R N}^{4}\left(\tau_{0}-\right. & \left.\tau_{k}^{-} \tan \varphi\right) K i_{3}\left(\frac{\tau_{k}^{-}}{\cos \varphi}\right) \sin \varphi d \varphi \\
& =T_{R N, M-1}^{4} \int_{\varphi=0}^{\bar{\varphi}_{M-1}^{-}} K i_{3}\left(\frac{\tau_{k}^{-}}{\cos \varphi}\right) \sin \varphi d \varphi+\sum_{m=2}^{M-2} T_{R N, m}^{4} \int_{\varphi=\widetilde{\varphi}_{m}^{+}}^{\bar{\varphi}_{m}^{-}} K i_{3}\left(\frac{\tau_{k}^{-}}{\cos \varphi}\right) \sin \varphi d \varphi
\end{aligned}
$$

where the boundary angles $\bar{\varphi}_{m}^{-}$and $\bar{\varphi}_{m}^{+}$are similarly defined by $\frac{H-h}{2}-\left(H-\hat{y}_{k}\right) \tan \bar{\varphi}_{m}^{-}=\hat{x}_{m}-\frac{\Delta \hat{x}}{2}$ and $\frac{H-h}{2}-\left(H-\hat{y}_{k}\right) \tan \bar{\varphi}_{m}^{+}=\hat{x}_{m}+\frac{\Delta \hat{x}}{2}$, with $\hat{x}_{m}<\frac{H-h}{2}$, from which (50) rewrites:

$$
\begin{aligned}
C_{N}(k)=\int_{\varphi=0}^{\xi_{N}^{+}} & T_{R N}^{4}\left(\tau_{0}-\tau_{k}^{-} \tan \varphi\right) K i_{3}\left(\frac{\tau_{k}^{-}}{\cos \varphi}\right) \sin \varphi d \varphi \\
& =T_{R N, M-1}^{4} \operatorname{Cis}_{3}\left(\tau_{k}^{-}, \bar{\varphi}_{M-1}^{-}\right)+\sum_{m=2}^{M-2} T_{R N, m}^{4}\left[\operatorname{Cis}_{3}\left(\tau_{k}^{-}, \bar{\varphi}_{m}^{-}\right)-\operatorname{Cis}_{3}\left(\tau_{k}^{-}, \bar{\varphi}_{m}^{+}\right)\right]
\end{aligned}
$$

whence $C_{N}(k)=\sum_{m=2}^{M-1} C_{k, m}^{N, \sigma_{O}} T_{R N, m}^{4}$ with $C_{k, m}^{N, \sigma_{O}}=C_{k, 2(M+P-1)-m}^{N, \sigma_{E}}$ for $2 \leq m \leq M-1$.

Obviously one similarly has $C_{S}(k)=\sum_{m=2}^{M-1} C_{k, m}^{S, \sigma_{O}} T_{R S, m}^{4}$ with $C_{k, m}^{S, \sigma_{O}}=C_{k, 2(M+P-1)-m}^{S, \sigma_{E}}$ for $2 \leq m \leq M-1$. Then the obstacle's western radiosity temperature is completely deduced from the obstacle's eastern radiosity temperature.

For the obstacle's northern radiosity temperature, using the formal substitution $\hat{y} \leftrightarrow \hat{x}$, one writes as for the eastern one: 


$$
\frac{\pi\left[T_{R \sigma_{N}}^{4}\left(\hat{x}_{k}\right)-\varepsilon_{\sigma_{N}} T_{\sigma_{N}}^{4}\right]}{2 \rho_{\sigma_{N}}}=\sum_{m=2}^{2(M+P-2)}\left(C_{k, m}^{N, \sigma_{N}} T_{R N, m}^{4}+C_{k, m}^{E, \sigma_{N}} T_{R E, m}^{4}+C_{k, m}^{O, \sigma_{N}} T_{R O, m}^{4}\right)+\kappa \aleph_{\sigma_{N}}\left(\hat{x}_{k}\right)
$$

with $C_{k, m}^{N, \sigma_{N}}=C_{k, m}^{E, \sigma_{E}}, C_{k, m}^{E, \sigma_{N}}=C_{k, m}^{N, \sigma_{E}}$ and $C_{k, m}^{O, \sigma_{N}}=C_{k, m}^{S, \sigma_{E}}$ for a given index $k$.

The obstacle's southern radiosity temperature is similarly obtained with the analogous coefficients $C_{k, m}^{S, \sigma_{S}}=C_{k, m}^{O, \sigma_{O}}=C_{k, m}^{E, \sigma_{E}}, C_{k, m}^{E, \sigma_{S}}=C_{k, m}^{N, \sigma_{O}}=C_{k, 2(M+P-1)-m}^{N, \sigma_{E}}$ and $C_{k, m}^{O, \sigma_{S}}=C_{k, m}^{S, \sigma_{O}}=C_{k, 2(M+P-1)-m}^{S, \sigma_{E}}$

\section{III-2. The cavity's radiosity temperatures}

Similarly to what precedes, the cavity's eastern surface radiosity temperature can be discretized in a same way and writes in the general case:

$$
\begin{aligned}
\frac{\pi\left[T_{R E}^{4}\left(\hat{y}_{k}\right)-\varepsilon_{E} T_{E}^{4}\right]}{2 \rho_{E}} & \\
= & \sum_{\substack{m=2 \\
M+2 P-3}}^{2(M+P-2)}\left(C_{k, m}^{O, E} T_{R O, m}^{4}+C_{k, m}^{N, E} T_{R N, m}^{4}+C_{k, m}^{S, E} T_{R S, m}^{4}\right) \\
& \left.+\sum_{m=M+1}^{\sigma_{k, m}, E} T_{R \sigma_{E}, m}^{4}+C_{k, m}^{\sigma_{S}, E} T_{R \sigma_{S}, m}^{4}+C_{k, m}^{\sigma_{N}, E} T_{R \sigma_{N}, m}^{4}\right)+\kappa \aleph_{E}\left(\hat{y}_{k}\right)
\end{aligned}
$$

where the coefficients $C_{k, m}^{S, E}$ are detailed in the Annex 3, some of them being of 0 value, depending on which case is treated.

For an identical position $\hat{y}_{k}$, the cavity's western surface radiosity temperature similarly writes:

$$
\begin{aligned}
\frac{\pi\left[T_{R O}^{4}\left(\hat{y}_{k}\right)-\varepsilon_{O} T_{O}^{4}\right]}{2 \rho_{O}} & \\
= & \sum_{\substack{m=2 \\
M+2 P-3}}^{2(M+P-2)}\left(C_{k, m}^{E, O} T_{R E, m}^{4}+C_{k, m}^{N, O} T_{R N, m}^{4}+C_{k, m}^{S, O} T_{R S, m}^{4}\right) \\
& +\sum_{m=M+1}^{\sigma_{O}, O}\left(C_{k, m}^{\sigma_{0}} T_{R \sigma_{O}, m}^{4}+C_{k, m}^{\sigma_{S}, O} T_{R \sigma_{S}, m}^{4}+C_{k, m}^{\sigma_{N}, O} T_{R \sigma_{N}, m}^{4}\right)+\kappa \aleph_{O}\left(\hat{y}_{k}\right)
\end{aligned}
$$

where $C_{k, m}^{E, O}=C_{k, m}^{O, E}$ and $C_{k, m}^{\sigma_{O}, O}=C_{k, m}^{\sigma_{E}, E}, C_{k, m}^{N, O}=C_{k, 2(M+P-1)-m}^{N, E}$ and $C_{k, m}^{S, O}=C_{k, 2(M+P-1)-m}^{S, E}$, $C_{k, m}^{\sigma_{S}, O}=C_{k, 2(M+P-1)-m}^{\sigma_{S}, E}$ and $C_{k, m}^{\sigma_{N}, O}=C_{k, 2(M+P-1)-m}^{\sigma_{N}, E}$, where the substitution $\hat{y} \leftrightarrow H-\hat{y}$ has been applied. Using the formal substitution $\hat{y} \leftrightarrow \hat{x}$ allows writing for the cavity's northern surface radiosity temperature:

$$
\begin{aligned}
\frac{\pi\left[T_{R N}^{4}\left(\hat{x}_{k}\right)-\varepsilon_{N} T_{N}^{4}\right]}{2 \rho_{N}} & \\
= & \sum_{m=2}^{2(M+P-2)}\left(C_{k, m}^{E, N} T_{R E, m}^{4}+C_{k, m}^{O, N} T_{R O, m}^{4}+C_{k, m}^{S, N} T_{R S, m}^{4}\right) \\
& +\sum_{m=M+1}^{M+2 P-3}\left(C_{k, m}^{\sigma_{O}, N} T_{R \sigma_{O}, m}^{4}+C_{k, m}^{\sigma_{N}, N} T_{R \sigma_{N}, m}^{4}+C_{k, m}^{\sigma_{E}, N} T_{R \sigma_{E}, m}^{4}\right)+\kappa \aleph_{N}\left(\hat{x}_{k}\right)
\end{aligned}
$$


with $C_{k, m}^{S, N}=C_{k, m}^{O, E}, C_{k, m}^{O, N}=C_{k, m}^{S, E}$ and $C_{k, m}^{E, N}=C_{k, m}^{N, E}$ for the cavity's surfaces, and $C_{k, m}^{\sigma_{N}, N}=C_{k, m}^{\sigma_{E}, E}$, $C_{k, m}^{\sigma_{o}, N}=C_{k, m}^{\sigma_{S}, E}$ and $C_{k, m}^{\sigma_{E}, N}=C_{k, m}^{\sigma_{N}, E}$ for the obstacle's surfaces.

The cavity's southern radiosity temperature similarly writes:

$$
\begin{aligned}
\frac{\pi\left[T_{R S}^{4}\left(\hat{x}_{k}\right)-\varepsilon_{S} T_{S}^{4}\right]}{2 \rho_{S}} & \\
= & \sum_{m=2}^{2(M+P-2)}\left(C_{k, m}^{E, S} T_{R E, m}^{4}+C_{k, m}^{O, S} T_{R O, m}^{4}+C_{k, m}^{N, S} T_{R N, m}^{4}\right) \\
& +\sum_{m=M+1}^{M+2 P-3}\left(C_{k, m}^{\sigma_{O}, S} T_{R \sigma_{O}, m}^{4}+C_{k, m}^{\sigma_{S}, S} T_{R \sigma_{S}, m}^{4}+C_{k, m}^{\sigma_{E}, S} T_{R \sigma_{E}, m}^{4}\right)+\kappa \aleph_{S}\left(\hat{x}_{k}\right)
\end{aligned}
$$

with $C_{k, m}^{O, S}=C_{k, 2(M+P-1)-m}^{S, E}, C_{k, m}^{N, S}=C_{k, m}^{O, E}$ and $C_{k, m}^{E, S}=C_{k, 2(M+P-1)-m}^{N, E}$ for the cavity's surfaces, and $C_{k, m}^{\sigma_{S}, S}=C_{k, m}^{\sigma_{E}, E}, C_{k, m}^{\sigma_{O}, S}=C_{k, 2(M+P-1)-m}^{\sigma_{S}, E}$ and $C_{k, m}^{\sigma_{E}, S}=C_{k, 2(M+P-1)-m}^{\sigma_{N}, E}$ for the obstacle's surfaces.

Note that for a given set of cavity's surfaces radiosity temperatures, the set of obstacle's radiosity temperatures can be written in a linear matrix system form:

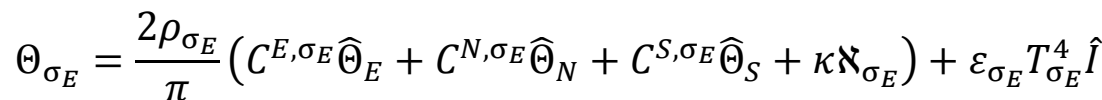

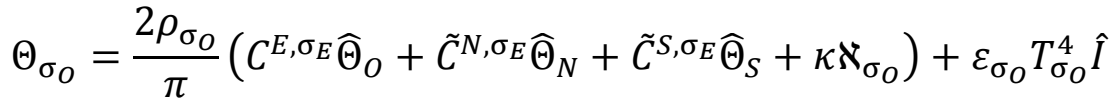

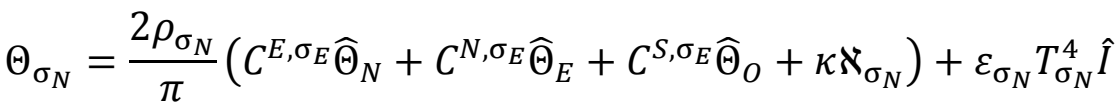

$$
\begin{aligned}
& \Theta_{\sigma_{S}}=\frac{2 \rho_{\sigma_{S}}}{\pi}\left(C^{E, \sigma_{E}} \widehat{\Theta}_{S}+\tilde{C}^{N, \sigma_{E}} \widehat{\Theta}_{E}+\tilde{C}^{S, \sigma_{E}} \widehat{\Theta}_{O}+\kappa \aleph_{\sigma_{S}}\right)+\varepsilon_{\sigma_{S}} T_{\sigma_{S}}^{4} \hat{I}
\end{aligned}
$$

where the $C^{E(N, S), \sigma_{E}}, \tilde{C}^{N(S), \sigma_{E}}$ are rectangular matrices of size $(2 P-1 ; 2 M+2 P-5)$, the $\Theta_{\sigma_{E(O, N, S)}}$ and $\aleph_{\sigma}$ are column vectors of size $2 P-1, \hat{I}$ being the unit column vector of same size, and the $\widehat{\Theta}_{E(O, N, S)}$ are column vectors of size $2 M+2 P-5$

Similarly, one forms a linear system of the four cavity's surfaces radiosity temperatures vectors as:

$$
\begin{gathered}
\widehat{\Theta}_{E}-\frac{2 \rho_{E}}{\pi}\left(\hat{C}^{O, E} \widehat{\Theta}_{O}+\hat{C}^{N, E} \widehat{\Theta}_{N}+\hat{C}^{S, E} \widehat{\Theta}_{S}\right) \\
=\frac{2 \rho_{E}}{\pi}\left(\bar{C}^{\sigma_{E}, E} \Theta_{\sigma_{E}}+\bar{C}^{\sigma_{S}, E} \Theta_{\sigma_{S}}+\bar{C}^{\sigma_{N}, E} \Theta_{\sigma_{N}}+\kappa \aleph_{E}\right)+\varepsilon_{E} T_{E}^{4} \bar{I} \\
\widehat{\Theta}_{O}-\frac{2 \rho_{O}}{\pi}\left(\hat{C}^{O, E} \widehat{\Theta}_{E}+\hat{\tilde{C}}^{N, E} \widehat{\Theta}_{N}+\hat{\tilde{C}}^{S, E} \widehat{\Theta}_{S}\right) \\
=\frac{2 \rho_{O}}{\pi}\left(\bar{C}^{\sigma_{E}, E} \Theta_{\sigma_{O}}+\overline{\tilde{C}}^{\sigma_{S}, E} \Theta_{\sigma_{S}}+\overline{\tilde{C}}^{\sigma_{N}, E} \Theta_{\sigma_{N}}+\kappa \aleph_{O}\right)+\varepsilon_{O} T_{O}^{4} \bar{I} \\
\widehat{\Theta}_{N}-\frac{2 \rho_{N}}{\pi}\left(\hat{C}^{N, E} \widehat{\Theta}_{E}+\hat{C}^{S, E} \widehat{\Theta}_{O}+\hat{C}^{O, E} \widehat{\Theta}_{S}\right) \\
=\frac{2 \rho_{N}}{\pi}\left(\bar{C}^{\sigma_{S}, E} \Theta_{\sigma_{O}}+\bar{C}^{\sigma_{E}, E} \Theta_{\sigma_{N}}+\bar{C}^{\sigma_{N}, E} \Theta_{\sigma_{E}}+\kappa \aleph_{S}\right)+\varepsilon_{N} T_{N}^{4} \bar{I}
\end{gathered}
$$




$$
\begin{aligned}
\widehat{\Theta}_{S}-\frac{2 \rho_{S}}{\pi}\left(\hat{\tilde{C}}^{N, E} \widehat{\Theta}_{E}+\hat{\tilde{C}}{ }^{S, E} \widehat{\Theta}_{O}+\hat{C}^{O, E} \widehat{\Theta}_{N}\right) \\
=\frac{2 \rho_{S}}{\pi}\left(\overline{\tilde{C}}^{\sigma, E} \Theta_{\sigma_{O}}+\bar{C}^{\sigma_{E}, E} \Theta_{\sigma_{S}}+\overline{\tilde{C}}^{\sigma_{N}, E} \Theta_{\sigma_{E}}+\kappa \aleph_{S}\right)+\varepsilon_{S} T_{S}^{4} \bar{I}
\end{aligned}
$$

where the $\hat{C}^{O(N, S), E}, \hat{\tilde{C}}^{N(S), E}$ are square matrices of size $(2 M+2 P-5 ; 2 M+2 P-5)$, the $\bar{C}^{\sigma_{E(N, S)}, E}$ and $\overline{\tilde{C}}^{\sigma_{S(N)}, E}$ are rectangular matrices of size $(2 M+2 P-5 ; 2 P-1), \aleph$ are column vectors of size $2 M+2 P-5$ and $\bar{I}$ the unit column vector of same size.

We shall now present some numerical results for various cases.

\section{IV - NUMERICAL RESULTS}

\section{IV-1. Grid generation}

The grid has been built considering a total number of cells (the 5 point cells included) depending on the number of cells in the area $\left[\frac{H-h}{2}, \frac{H+h}{2}\right]$ and outside, such that $N_{t}=2 M+2 P-3$ where $M-2$ is the number of non-zero depth cells on the length $\frac{H-h}{2}$.

Assuming cells intervals of constant depth in the whole domain leads to $\Delta x=\frac{H}{N_{t}-5}$, and in the area $\left[0, \frac{H-h}{2}\right]$, the interval $\Delta x$ must verify $(M-2) \Delta x=\frac{H-h}{2}$, whence $M=2+\llbracket \frac{H-h}{2 \Delta x} \rrbracket$, or in an equivalent way $M=2+\llbracket \frac{H-h}{H}(M+P-4) \rrbracket$, where $\llbracket x \rrbracket$ stands for the integer part of a real number $x$.

Note that if it exists two integers $q$ and $r$ such that $\frac{h}{H}=1-\frac{q}{r}$, and if it exists an integer $m$ which verifies $N_{t}-5=2 m r$, the integer $M$ is defined by the simple relation $M=2+m q$, whence $P=2+m(r-q)$ : under these two conditions, the interval $\Delta x$ is perfectly determined and each non-zero depth cell's centre is distant from its neighbour of $\Delta x$ everywhere on a cavity's surface and on an obstacle's surface.

If however the ratio $\frac{h}{H}$ is not rational, although a depth interval $\Delta x$ can be defined from $\Delta x=\frac{H}{N_{t}-5}$ and the integer $M$ from $M=2+\llbracket \frac{H-h}{2 \Delta x} \rrbracket$, the latter relation cannot insure that the nodes position defined by the relation $(E)$ with a same depth interval will be verified. To overcome this difficulty, we define two depth intervals on the areas $\left[\frac{H-h}{2}, \frac{H+h}{2}\right]$ and elsewhere, such that $\Delta x_{C}=\frac{H-h}{2(M-2)}$, the integers $M$ and $P$ still being defined by $M=2+\llbracket \frac{H-h}{2 \Delta x} \rrbracket$ with $\Delta x=\frac{H}{N_{t}-5}$ a fictive depth interval, and $P=\frac{N_{t}+3}{2}-M$ leading to an obstacle's depth interval $\Delta x_{O}=\frac{h}{2(P-2)}$

With such a definition, one always has $N_{t}=2 M+2 P-3$ and $\Delta x_{C} \approx \Delta x_{O}$, the equality being strictly verified for obstacles such that the ratio $\frac{h}{H}$ is rational and for a judicious total cells number (punctual cells included); on the contrary, a special attention must be paid for the nodes closed to the obstacle's boundaries $\{M-1, M+1,2 M+2 P-3,2 M+2 P-1\}$

With such a description, any point $\tilde{x}$ on a cavity's surface is located in a cell characterised by its integer index $p$ defined by the useful relations:

if $\tilde{x}<\frac{H-h}{2}, p=2+\llbracket \frac{\tilde{x}}{\Delta x_{C}} \rrbracket$; if $\tilde{x}=\frac{H-h}{2}, p=M$; if $\frac{H-h}{2}<\tilde{x}<\frac{H}{2}, p=M+1+\llbracket \frac{\tilde{x}-\frac{H-h}{2}}{\Delta x_{O}} \rrbracket$

if $\tilde{x}=\frac{H}{2}, p=M+P-1$; if $\frac{H}{2}<\tilde{x}<\frac{H+h}{2}, p=M+P+\llbracket \frac{\tilde{x}-\frac{H}{2}}{\Delta x_{O}} \rrbracket$;if $\tilde{x}=\frac{H+h}{2}, p=M+2 P-2$; 
and if $\tilde{x}>\frac{H+h}{2}, p=M+2 P-1+\llbracket \frac{\tilde{x}-\frac{H+h}{2}}{\Delta x_{C}} \rrbracket$

The previous relations reduce to $p=3+\llbracket \frac{\tilde{x}}{\Delta x} \rrbracket$ if $\frac{H}{2}<\tilde{x}<\frac{H+h}{2}, p=4+\llbracket \frac{\tilde{x}}{\Delta x} \rrbracket$ if $\frac{H}{2}<\tilde{x}<\frac{H+h}{2}$, and finally $p=5+\llbracket \frac{\tilde{x}}{\Delta x} \rrbracket$ if $\tilde{x}>\frac{H+h}{2}$ for the case $\Delta x_{C}=\Delta x_{O}$

\section{IV-2. Radiosity temperatures description for different varying parameters}

The following results are obtained on a Personal Computer with 3.25 GB of RAM from numerical programs written in Fortran 77. The special functions $\mathrm{Ki}_{2}, \mathrm{Bis}_{3}$ and $\mathrm{Cis}_{3}$ are computed from the numerical routines written by Altac and Tekkalmaz and D. E. Amos [28].

Since the cavity's and obstacle's radiosity temperatures are coupled each other, the computation program is built in an iterative way with an error criterion such that the condition $\left|T_{R}^{(n+1)}-T_{R}^{(n)}\right|<10^{-6} K$ is verified for all radiosity temperatures $T_{R}$ on the eight boundary surfaces in each small surface cell labelled by its index $k$. Note that the accuracy from this criterion is only relative, since the medium is not at radiative equilibrium but at imposed temperature, for which the condition $\operatorname{div} \boldsymbol{q}_{r}=0$ is not verified, where $\boldsymbol{q}_{\boldsymbol{r}}$ is the radiative flux vector internal field. Hence the integrated condition $\sum_{i} \boldsymbol{n}_{i} \int_{l_{i}} \boldsymbol{q}_{\boldsymbol{r}} d l_{i}=0$ implying the normal integrated fluxes on the eight surfaces, which can define an absolute accuracy criterion as in [14], cannot be verified unless the complete radiative field is determined in the whole medium. Under this condition of relative accuracy, the selected criterion $\left|T_{R}^{(n+1)}-T_{R}^{(n)}\right|<10^{-6} \mathrm{~K}$ for the radiosity temperatures always converges in all selected cases for any cells number on the eight surfaces.

\section{IV.2.1. Isothermal semi-transparent media:}

We shall quantify now the influence of the obstacle on the cavity's radiosity temperatures, firstly when all the surfaces of the obstacle are black and the four surfaces of the cavity are reflecting the incident radiation with the same emissivity, the cavity containing a cold isothermal medium at $T_{m}=0 \mathrm{~K}$. We look simultaneously at the obstacle's size and absorption coefficient of the surrounding medium, for given surfaces temperatures.

To evaluate the performance of the numerical integrations and observe the influence of the grid's cells number, we choose first a black obstacle at high temperature with $\varepsilon_{O}=1$, while the almost perfectly reflecting surfaces of the cavity have their emissivity set to $\varepsilon_{C}=0.01$. The isothermal surfaces have their temperatures imposed to $T_{C}=100 \mathrm{~K}$ and $T_{O}=1000 \mathrm{~K}$. We let vary the length ratio from $\frac{h}{\mathrm{H}} \ll 1$ to high values around 1 for a given absorption coefficient for almost transparent media to moderately absorbing ones. We put an insight on the temperatures at the centre of the cavity's surfaces so as at the two values $\frac{H-h}{2}$ and $\frac{H+h}{2}$. Numerical results are reported in Figs. 4a-e. We also give an insight of the numerical values depending on the iterative process for a particular case in Table 1 for a small ratio $\frac{h}{H}=0.01$

- $\quad$ Case $\frac{h}{H}=0.01:\left(\frac{h}{H}<\sqrt{5}-2\right)$

\begin{tabular}{|c|c|c|c|c|}
\hline$\kappa\left(m^{-1}\right)$ & $N_{t},(M, P)$ & $N_{i t e r}$ & $T_{R}\left(\frac{H \pm h}{2}\right)(K)$ & $T_{R}\left(\frac{H}{2}\right)(K)$ \\
\hline 0.01 & $101(49,3)$ & 519 & 757.971 & 757.241 \\
\hline 0.01 & $251(123,4)$ & 520 & 759.131 & 759.124 \\
\hline 0.01 & $501(247,5)$ & 520 & 759.127 & 759.124 \\
\hline 0.01 & $1001(495,7)$ & 520 & 759.125 & 759.123 \\
\hline
\end{tabular}




\begin{tabular}{|c|c|c|c|c|}
\hline 0.01 & $2001(990,12)$ & 520 & 759.124 & 759.123 \\
\hline 0.01 & $3001(1485,17)$ & 520 & 759.1232 & 759.1232 \\
\hline 0.1 & $101(49,3)$ & 143 & 533.873 & 531.878 \\
\hline 0.1 & $251(123,4)$ & 143 & 536.055 & 536.029 \\
\hline 0.1 & $501(247,5)$ & 143 & 536.041 & 536.029 \\
\hline 0.1 & $1001(495,7)$ & 143 & 536.034 & 536.029 \\
\hline 0.1 & $2001(990,12)$ & 143 & 536.030 & 536.029 \\
\hline 0.1 & $3001(1485,17)$ & 143 & 536.0293 & 536.0292 \\
\hline 1. & $101(49,3)$ & 25 & 298.094 & 291.411 \\
\hline 1. & $251(123,4)$ & 25 & 304.472 & 304.385 \\
\hline 1. & $501(247,5)$ & 25 & 304.424 & 304.385 \\
\hline 1. & $1001(495,7)$ & 25 & 304.401 & 304.385 \\
\hline 1. & $2001(990,12)$ & 26 & 304.389 & 304.385 \\
\hline 1. & $3001(1485,17)$ & 26 & 304.3851 & 304.3853 \\
\hline 10. & $101(49,3)$ & 17 & 77.453 & 74.670 \\
\hline 10. & $251(123,4)$ & 19 & 80.105 & 80.077 \\
\hline 10. & $501(247,5)$ & 20 & 80.088 & 80.078 \\
\hline 10. & $1001(495,7)$ & 21 & 80.079 & 80.078 \\
\hline 10. & $2001(990,12)$ & 21 & 80.074 & 80.078 \\
\hline 10. & $3001(1485,17)$ & 22 & 80.0728 & 80.0779 \\
\hline
\end{tabular}

Table 1: Cavity's surface radiosity temperatures at three positions $\frac{H \pm h}{2}$ and $\frac{H}{2}$ for $\frac{h}{H}=0.01$
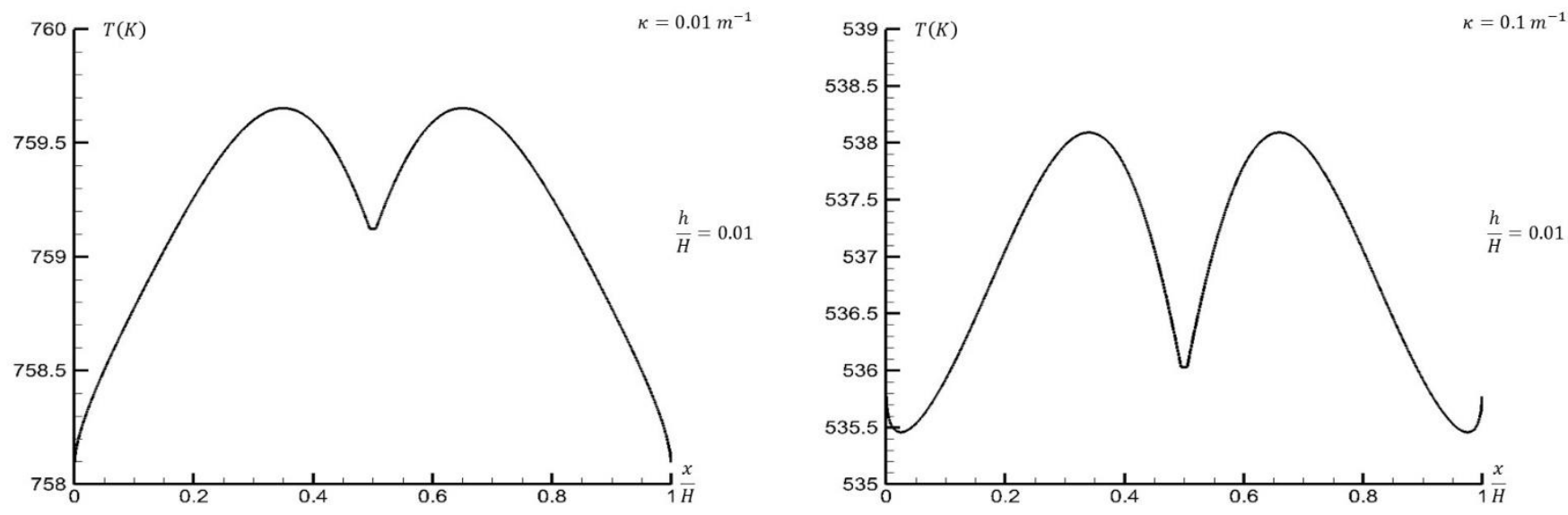

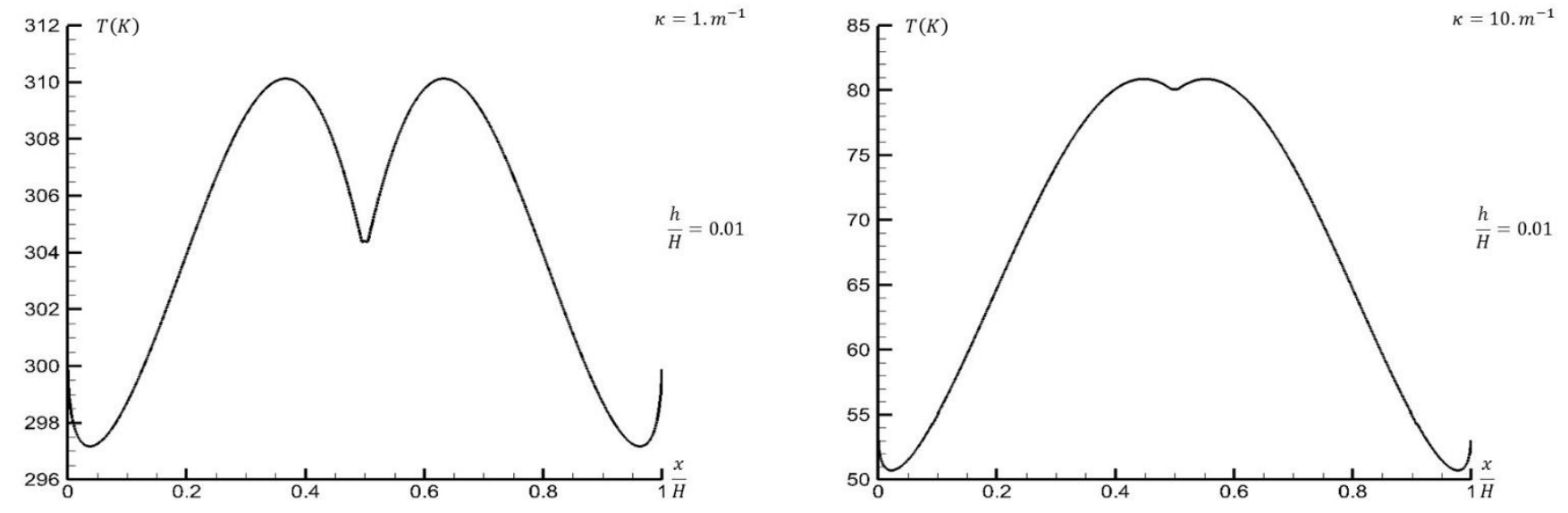

Figure 4a: Cavity's surface radiosity temperature for a small ratio $\frac{h}{H}=0.01$

- $\quad$ Case $\frac{h}{H}=0.1:\left(\frac{h}{H}<\sqrt{5}-2\right)$
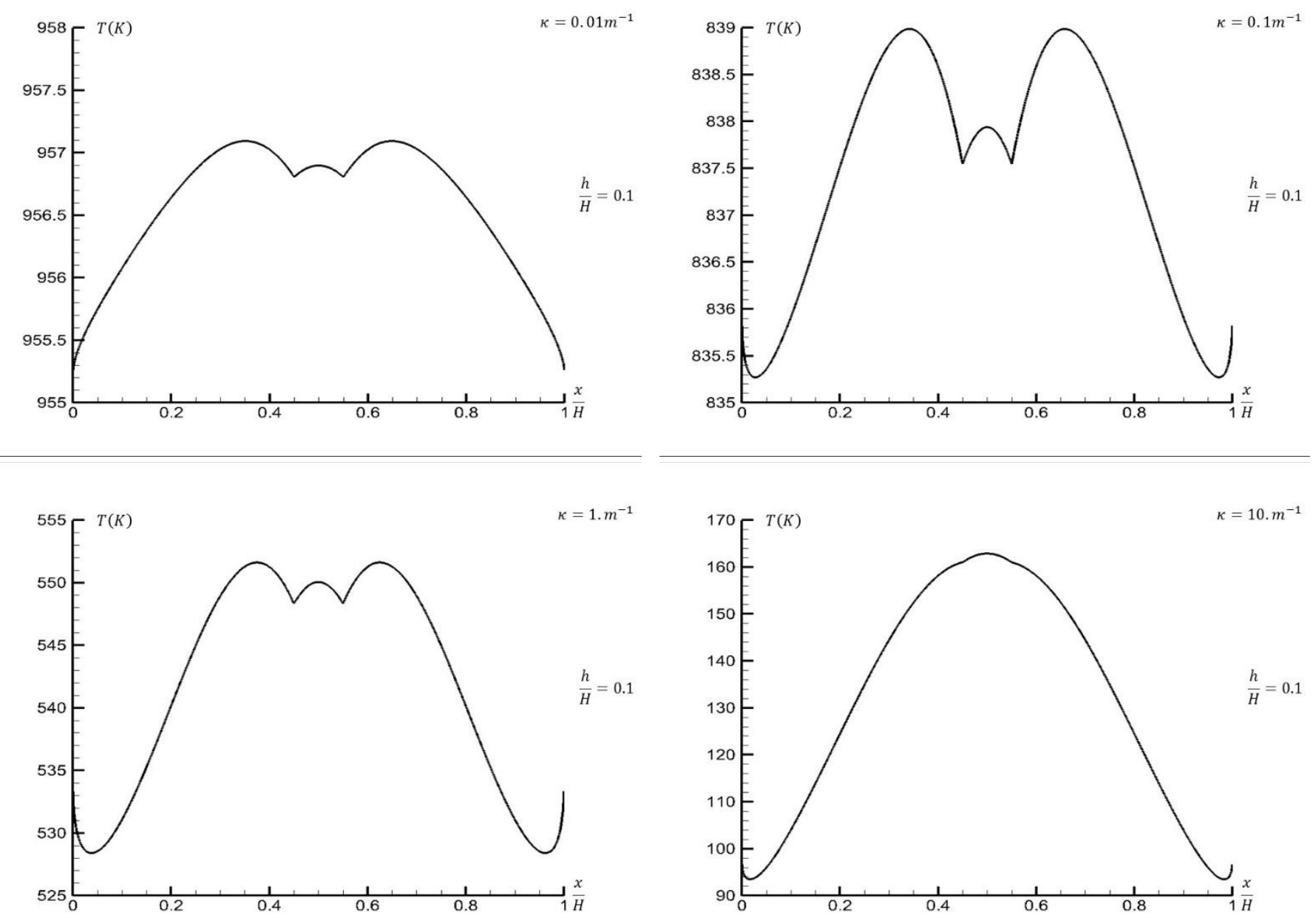

Figure 4b: Cavity's surface radiosity temperature for a ratio $\frac{h}{H}=0.1$ verifying $\frac{h}{H}<\sqrt{5}-2$

- $\quad$ Case $\frac{h}{H}=0.3:\left(\sqrt{5}-2<\frac{h}{H}<\frac{1}{3}\right)$ 

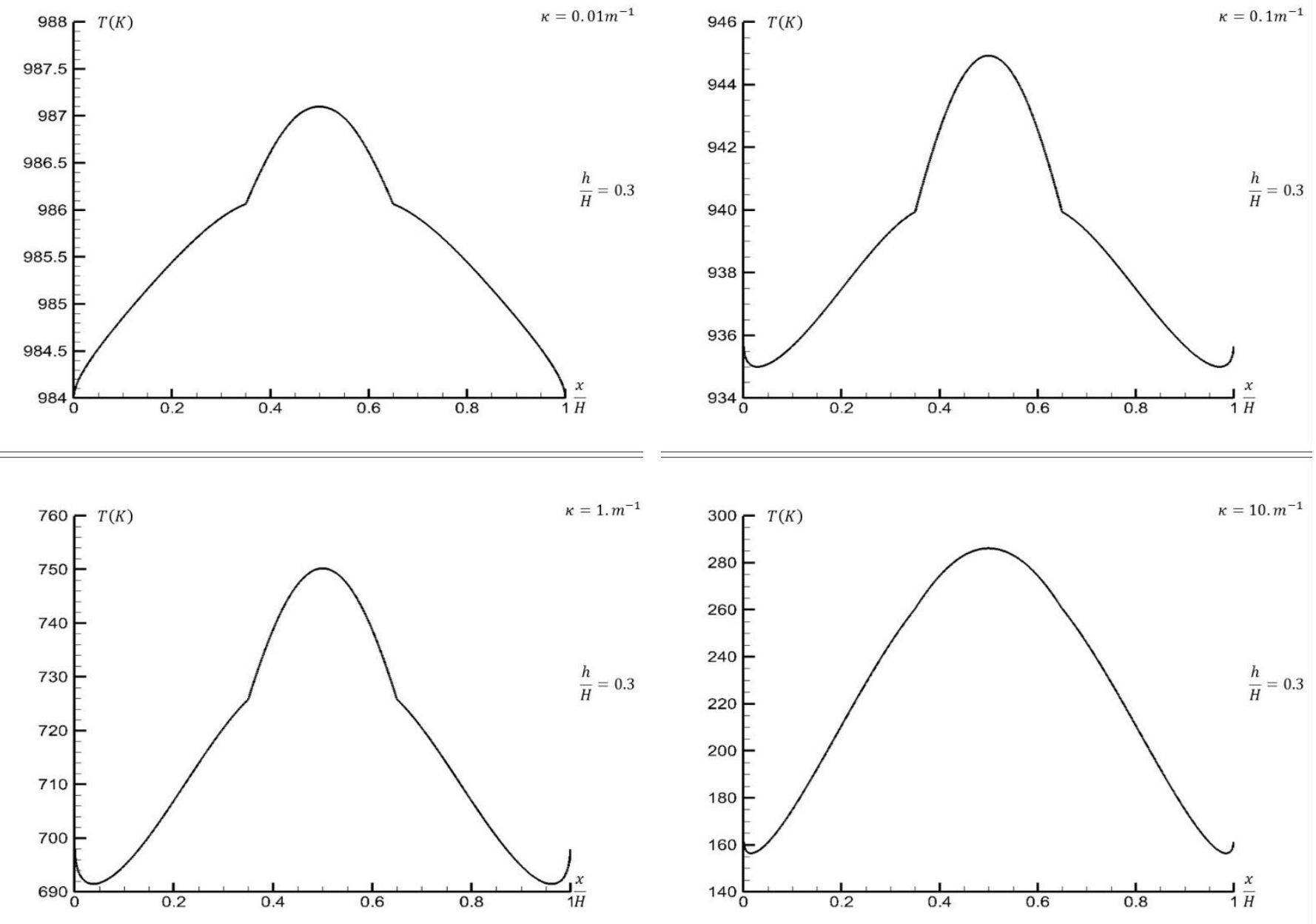

Figure 4c: Cavity's surface radiosity temperature for a ratio $\frac{h}{H}=0.3$ in the domain $] \sqrt{5}-2, \frac{1}{3}[$

- $\quad$ Case $\frac{h}{H}=0.5:\left(\frac{h}{H}>\frac{1}{3}\right)$
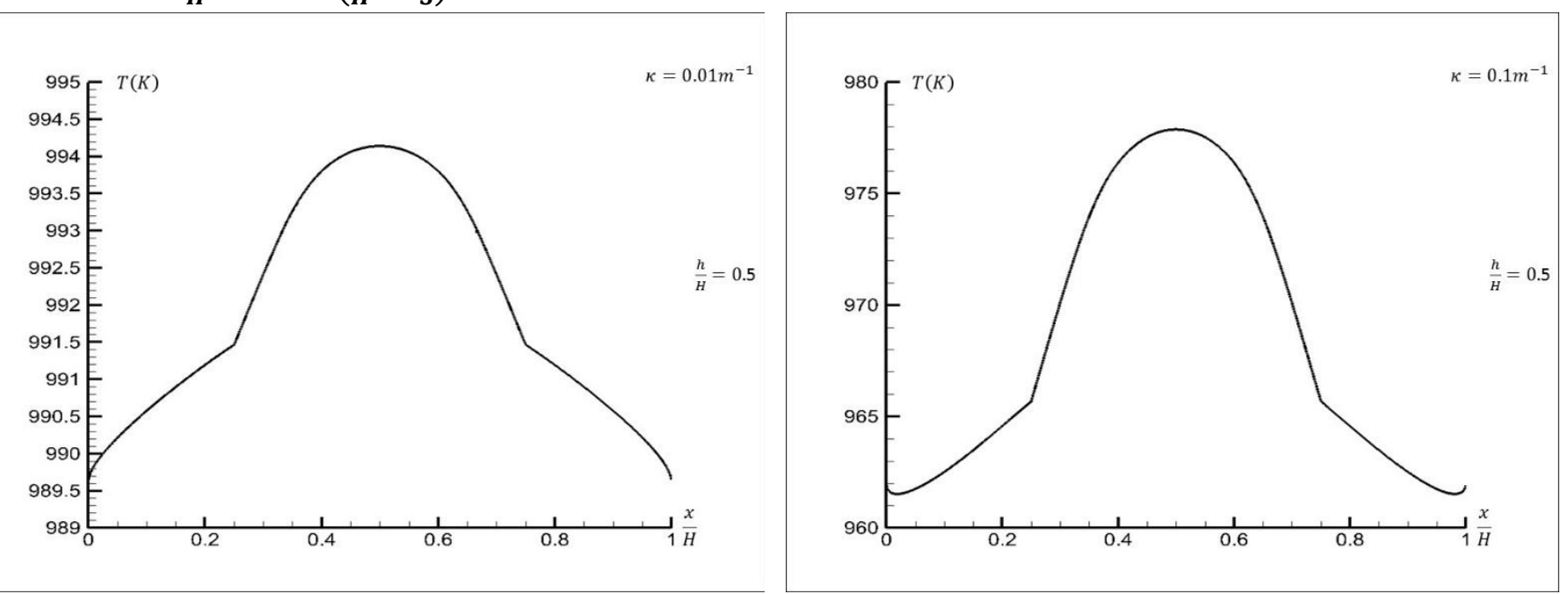

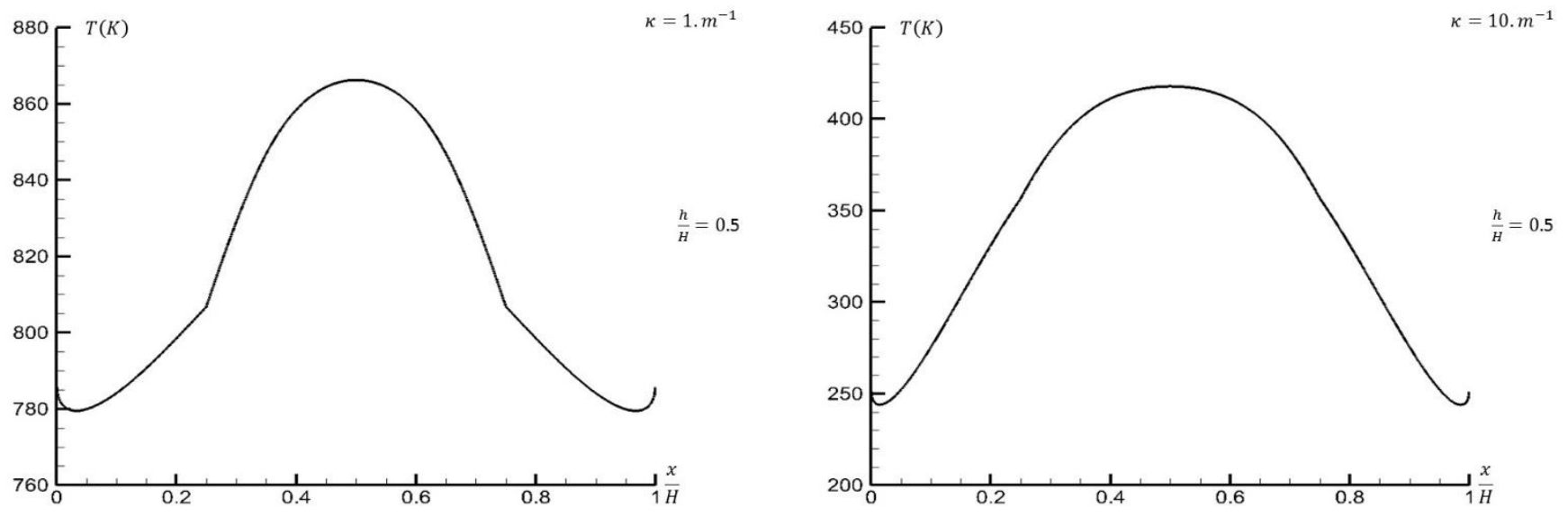

Figure 4d: Cavity's surface radiosity temperature for a ratio $\frac{h}{H}=0.5$ close to the limit $\frac{1}{3}$

- $\quad$ Case $\frac{h}{H}=0.9:\left(\frac{h}{H}>\frac{1}{3}\right)$
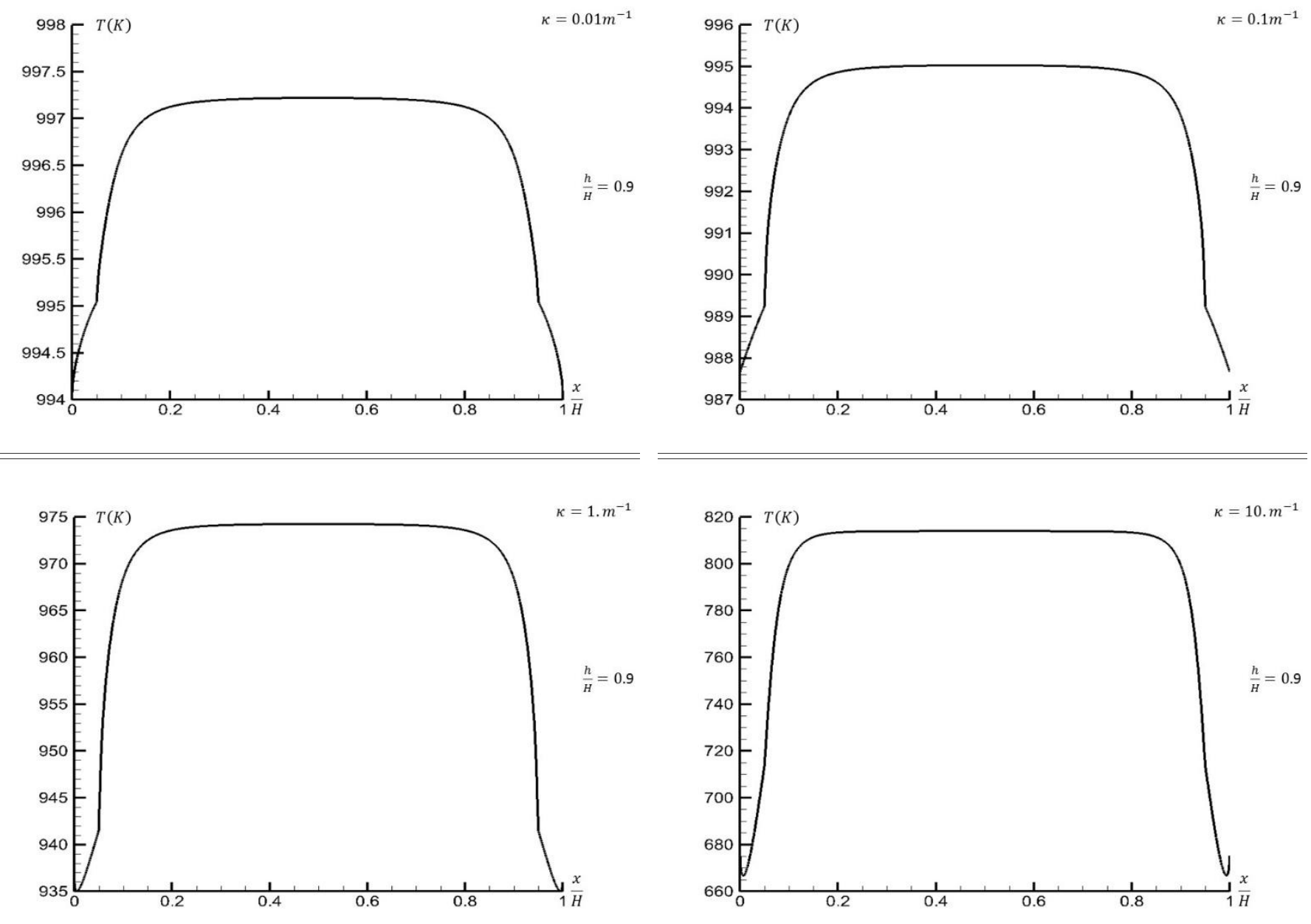

Figure 4e: Cavity's surface radiosity temperature for a high ratio $\frac{h}{H}=0.9$

The iterative resolution shows that the iterations number depends essentially on the absorption coefficient and not on the cells' number, excepted for moderate to high absorptions. In the case of almost transparent or few absorbing media, the results are qualitatively and quantitatively of good quality even for a small number of cells around $N_{t}=251$ for any ratio $\frac{h}{H}$, like for cavities without any obstacle, but 
increasing the cells number insures smooth results. The presented figures have been obtained for a total cells number $N_{t}=1001$ in each configuration, which allows short computation times and accurate results.

The highly different behaviours of the cavity's radiosity temperatures, depending on the ratio $\frac{h}{H}$ values, are significantly put in evidence in Figs (4a-e): when the ratio is smaller than $\sqrt{5}-2$, the influence of the obstacle is not limited to the area $\left[\frac{H-h}{2}, \frac{H+h}{2}\right]$ but strongly affects the whole extended zone $\left[\frac{2 h H}{H+h}, H \frac{H-h}{H+h}\right]$ which can be of large size up to moderate absorption coefficients. When the ratio increases, the influence of the obstacle is more concentrated in the domain $\left[\frac{H-h}{2}, \frac{H+h}{2}\right]$, while the morphology of the radiosity temperature changes in the obstacle's zone as soon as $\frac{h}{H}$ becomes larger than $\sqrt{5}-2$.

Note that for optically thin media with low absorption coefficients, the edge effects are not significant near the surface boundaries, while they significantly modify the radiosity temperature structure for moderate absorption coefficients, especially for small ratios $\frac{h}{H}$.

In the way to obtain the minimal obstacle's size which can be detected and still has an influence on the cavity's radiosity temperatures, we decrease the length of the obstacle's side while increasing the cells number, for a given absorption coefficient, by comparing with the radiosity temperature obtained without any obstacle.

For a square cavity of length $H=1 \mathrm{~m}$, whose four reflecting surfaces of same emissivity $\varepsilon_{C}=0.01$ are isothermal, with an imposed temperature $T_{C}=100 \mathrm{~K}$, the radiosity temperature on the surfaces without any internal obstacle for various absorption coefficients inside the cold medium of zero constant temperature is given by:

$$
\begin{aligned}
\frac{\pi\left[T_{R}^{4}\left(\hat{y}_{k}\right)-\varepsilon_{C} T_{C}^{4}\right]}{2 \rho_{C}} & \\
& =\int_{\varphi=0}^{\tan ^{-1}\left(\frac{H}{H-\hat{y}_{k}}\right)} T_{R}^{4}\left[H-\left(H-\hat{y}_{k}\right) \tan \varphi\right] K i_{3}\left[\frac{\kappa\left(H-\hat{y}_{k}\right)}{\cos \varphi}\right] \sin \varphi d \varphi \\
& +\int_{\varphi=0}^{\tan ^{-1}\left(\frac{H-\hat{y}_{k}}{H}\right)} T_{R}^{4}\left(\hat{y}_{k}+H \tan \varphi\right) K i_{3}\left(\frac{\kappa H}{\cos \varphi}\right) \cos \varphi d \varphi \\
& +\int_{\varphi=0}^{\tan ^{-1}\left(\frac{\hat{y}_{k}}{H}\right)} T_{R}^{4}\left(\hat{y}_{k}-H \tan \varphi\right) K i_{3}\left(\frac{\kappa H}{\cos \varphi}\right) \cos \varphi d \varphi \\
& +\int_{\varphi=0}^{\tan ^{-1}\left(\frac{H}{\hat{y}_{k}}\right)} T_{R}^{4}\left(H-\hat{y}_{k} \tan \varphi\right) K i_{3}\left(\frac{\kappa \hat{y}_{k}}{\cos \varphi}\right) \sin \varphi d \varphi
\end{aligned}
$$

In the case of very absorbing media, $\frac{T_{R}}{T_{C}} \rightarrow \sqrt[4]{\varepsilon_{C}} \approx 0.316$, and the radiosity temperature on each side of the cavity tends to be constant with $T_{R} \approx 31.62 \mathrm{~K}$. The symmetric radiosity temperatures relatively to $\frac{H}{2}$, without any obstacle, are numerically reported on Table 2 at the surface's centre and at the end points:

\begin{tabular}{|c|c|c|}
\hline$\kappa\left(m^{-1}\right)$ & $T_{R}(x \rightarrow 0 ; x \rightarrow H)(K)$ & $T_{R}\left(\frac{H}{2}\right)(K)$ \\
\hline 0.01 & 84.279 & 84.142 \\
\hline 0.1 & 56.613 & 55.748 \\
\hline 1. & 39.390 & 35.672 \\
\hline
\end{tabular}


Table 2: Cavity's surface radiosity temperatures without any obstacle at three positions $0, H$ and $\frac{H}{2}$

Note that from Eq. (70), the radiosity temperature is not constant on the surface in the most general case, even for same constant and equal surface temperatures, at any absorption coefficient, the radiosity temperature being constant only in the limiting case of the optically depth media approximation $\kappa \rightarrow \infty$ The evolution of the cavity surface radiosity temperature without any obstacle inside the cavity is depicted in Fig. 5 for several absorption coefficients when the four surfaces are isothermal $\left(T_{C}=100 \mathrm{~K}\right)$ with the same emissivity $\varepsilon_{C}=0.01$. One observes that the radiosity temperature is not constant but tends to a constant value when the absorption coefficient increases, close to $\sqrt[4]{\varepsilon_{C}} T_{C}$ for $\kappa=10 . m^{-1}$

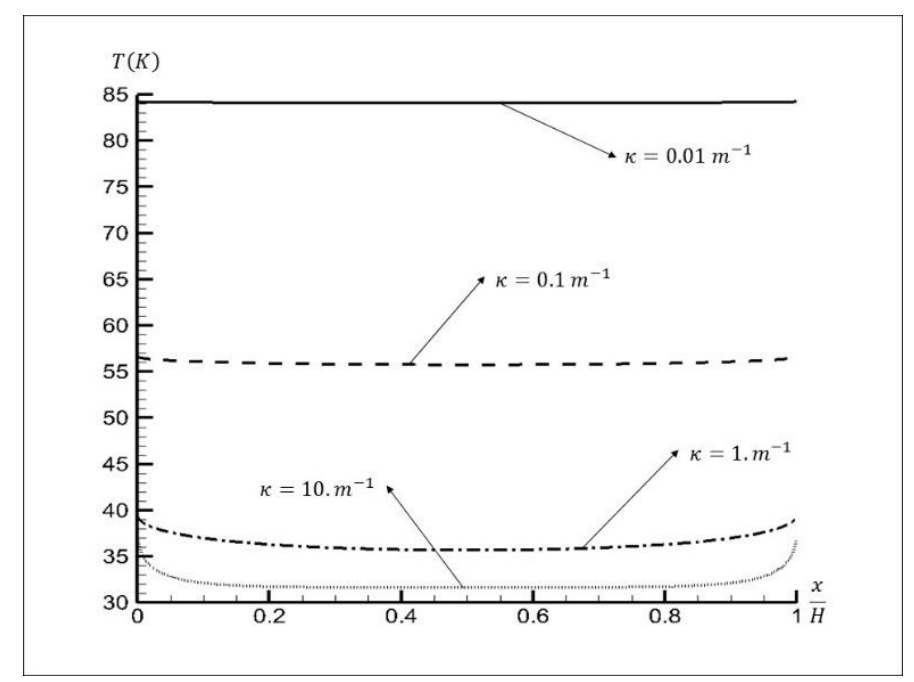

Figure 5: Cavity's surface radiosity temperature without any obstacle when $T_{C}=100 \mathrm{~K}$ for various absorption coefficients

If one compares to the radiosity temperatures obtained for a hot very small obstacle with $\frac{h}{H} \ll 1$, one observes that the obstacle remains playing a significant rule, strongly increasing the radiosity temperature mean magnitude and roughly modifying its morphologic structure in the case of optically thin media, while for relatively high optical depths such as $\kappa H=10$., the radiosity temperatures magnitude is not very far from the attempted value in a cavity without any obstacle in the same conditions of reflexion on the surfaces, although its structure is altered comparatively to the case with no internal obstacle.
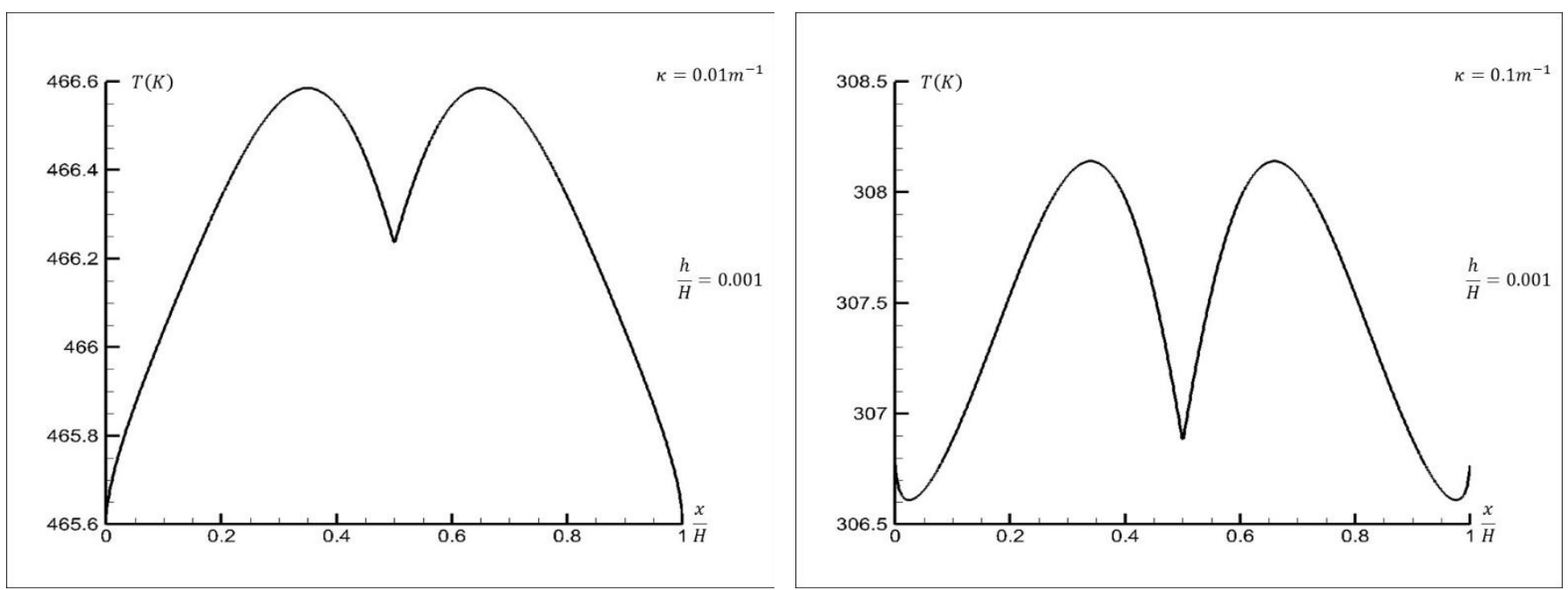

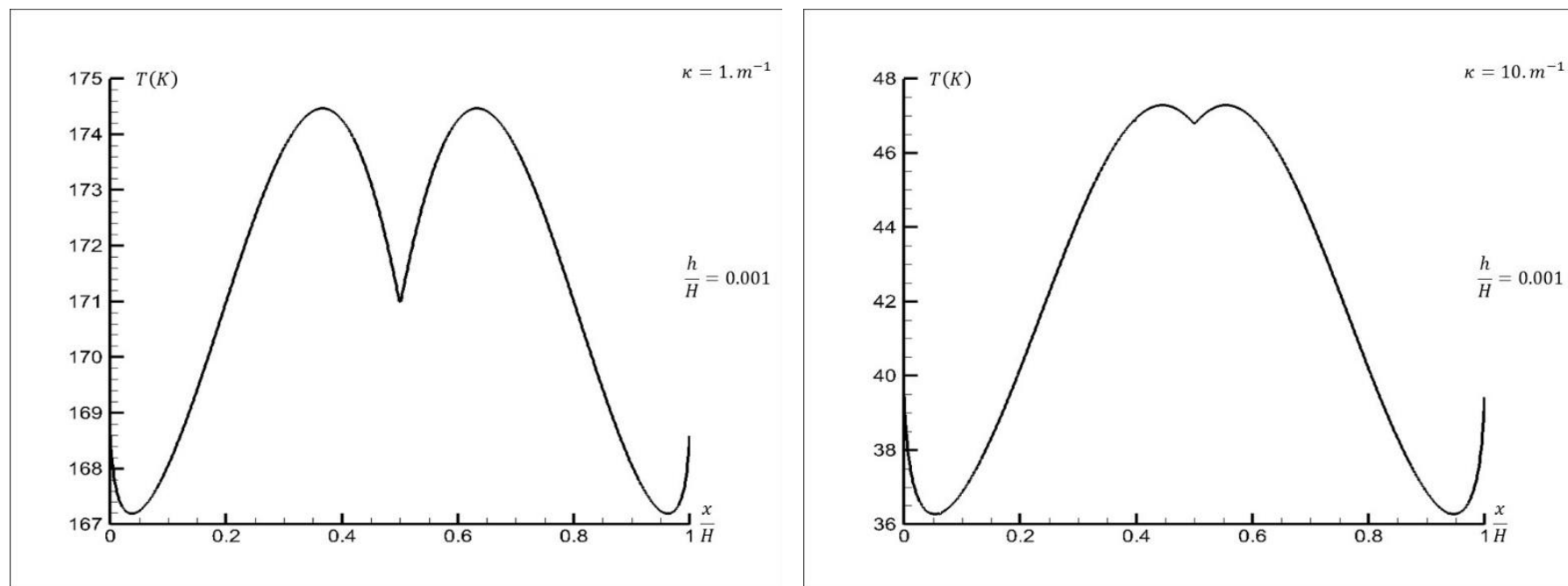

Figure 6: Cavity's surface radiosity temperature for a very small obstacle with $\frac{h}{H}=10^{-3}$

Indeed, the influence of the obstacle illustrated in Fig. 6 still remains noticeable on the cavity's radiosity temperature for the four selected absorption coefficients, with a qualitative behaviour very similar to the case $\frac{h}{H}=0.01$. For instance, when the semi-transparent medium is weakly absorbing with $\kappa=0.01 \mathrm{~m}^{-1}$, the mean radiosity temperature on one cavity's highly reflecting surface of temperature $T_{C}=100 \mathrm{~K}$ is around $T_{R C}=466 \mathrm{~K}$ for a hot black obstacle of temperature $T_{C}=1000 \mathrm{~K}$ with $\frac{h}{H}=10^{-3}$, while it is only $84 K$ when $\frac{h}{H}=0$.

For smaller obstacles with to $\frac{h}{H}<10^{-3}$, our results remain no longer accurate with oscillating values even for a large cells number. Nevertheless, very small obstacles up to $\frac{h}{H} \sim 10^{-3}$ can be taken into account and may be considered as a radiative perturbation on the whole device, but of small amplitude for relatively high absorption coefficients.

Interesting now again to the obstacle characterised by $\frac{h}{H}=0.01$, one inverts the temperatures of the cavity and black obstacle's surfaces, i.e. the obstacle is cold relatively to the cavity, and one examines the radiosity temperature behaviour for the same four selected absorption coefficients when the internal medium remains cold at $T_{m}=0 \mathrm{~K}$. The results are depicted in Fig. 7 for equivalent conditions of reflection on the surfaces of the cavity, i.e. $\varepsilon_{C}=0.01$
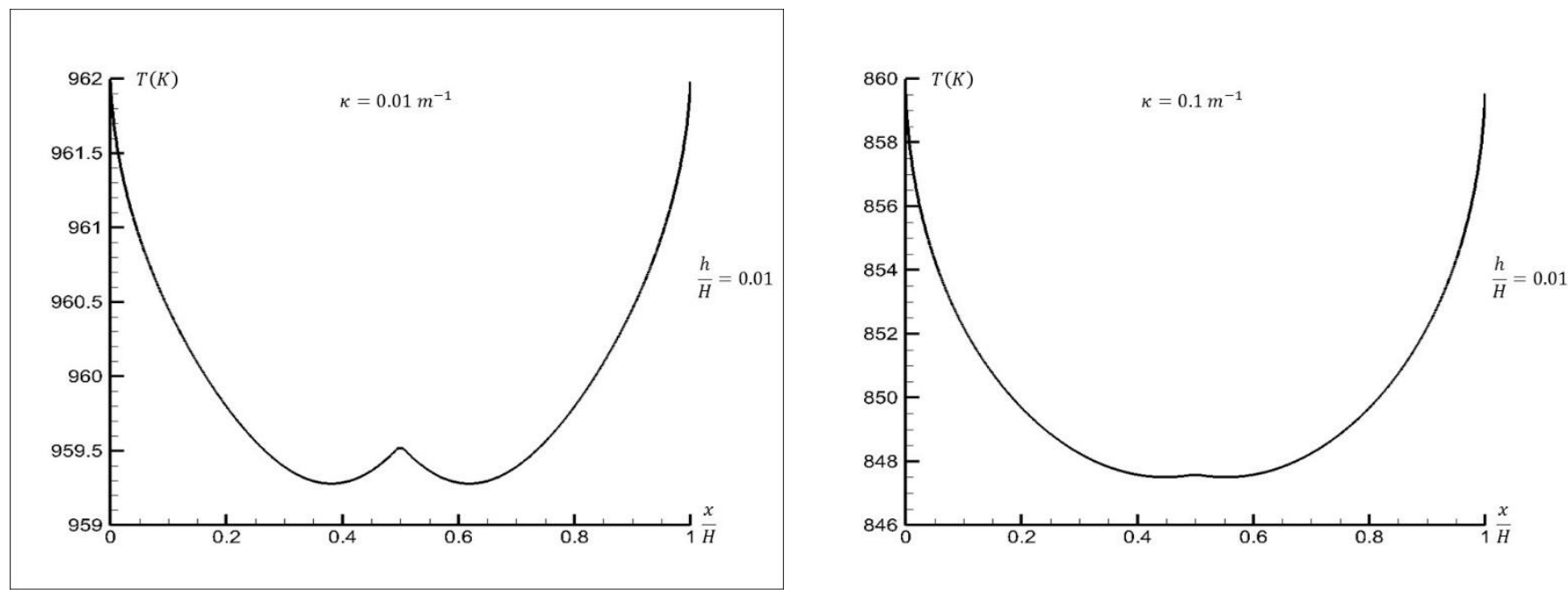

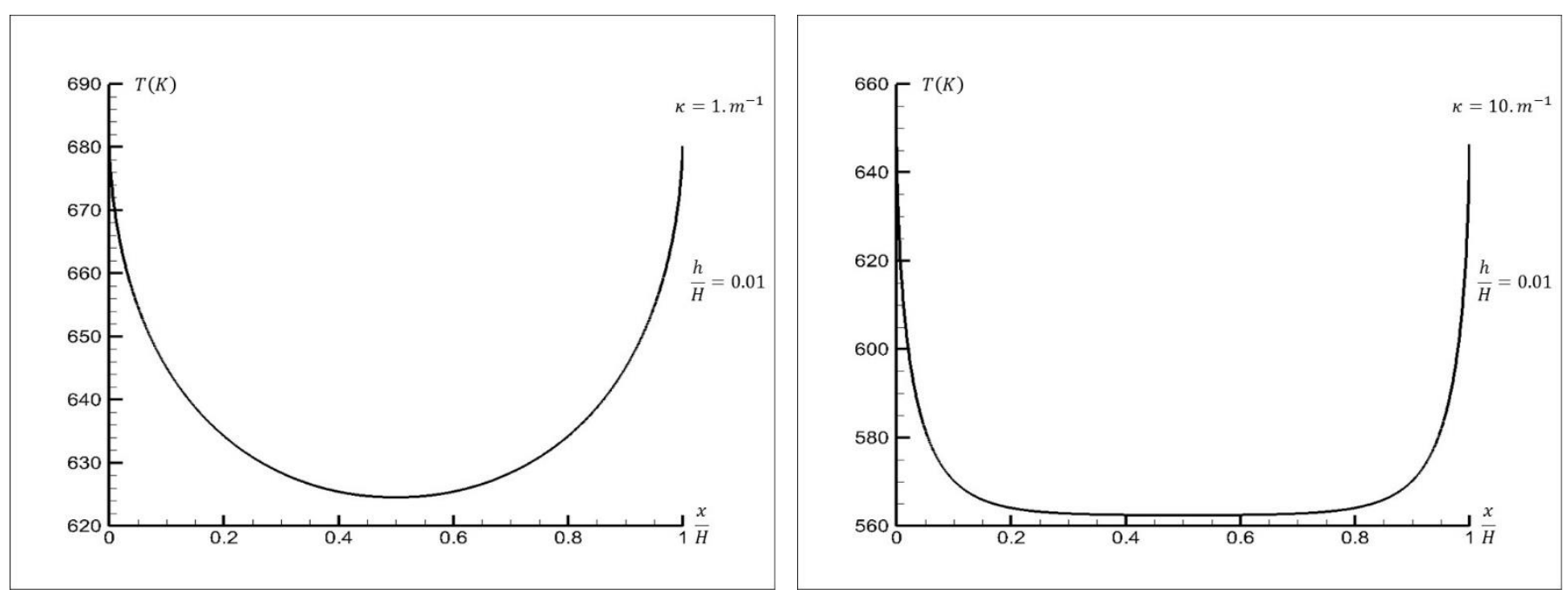

Figure 7: Cavity's surface radiosity temperature for $T_{C}=1000 \mathrm{~K}$ and $T_{O}=100 \mathrm{~K}$, case $\frac{h}{H}=0.01$

For a cold small obstacle, one observes that its influence on the cavity surface radiosity temperature is very limited to the case of an optically thin medium, with a small amplitude. As soon as the absorption coefficient increases, the radiosity temperature field on the cavity surface has a structure close to the one obtained when no obstacle is present, with a magnitude rapidly tending to the optically thick media radiosity temperature. One concludes that obstacles with small ratios $\frac{h}{H}$ have a strong influence on the cavity's radiosity temperature if they are much hotter than the cavity's surfaces.

Returning to the case of a hot obstacle, we look at the influence of the emissivity of the cavity's surfaces on the radiosity temperatures with respect to the absorption coefficient when the obstacle's size is much lower than the cavity's one. The radiosity temperatures are reported in the Fig. 8
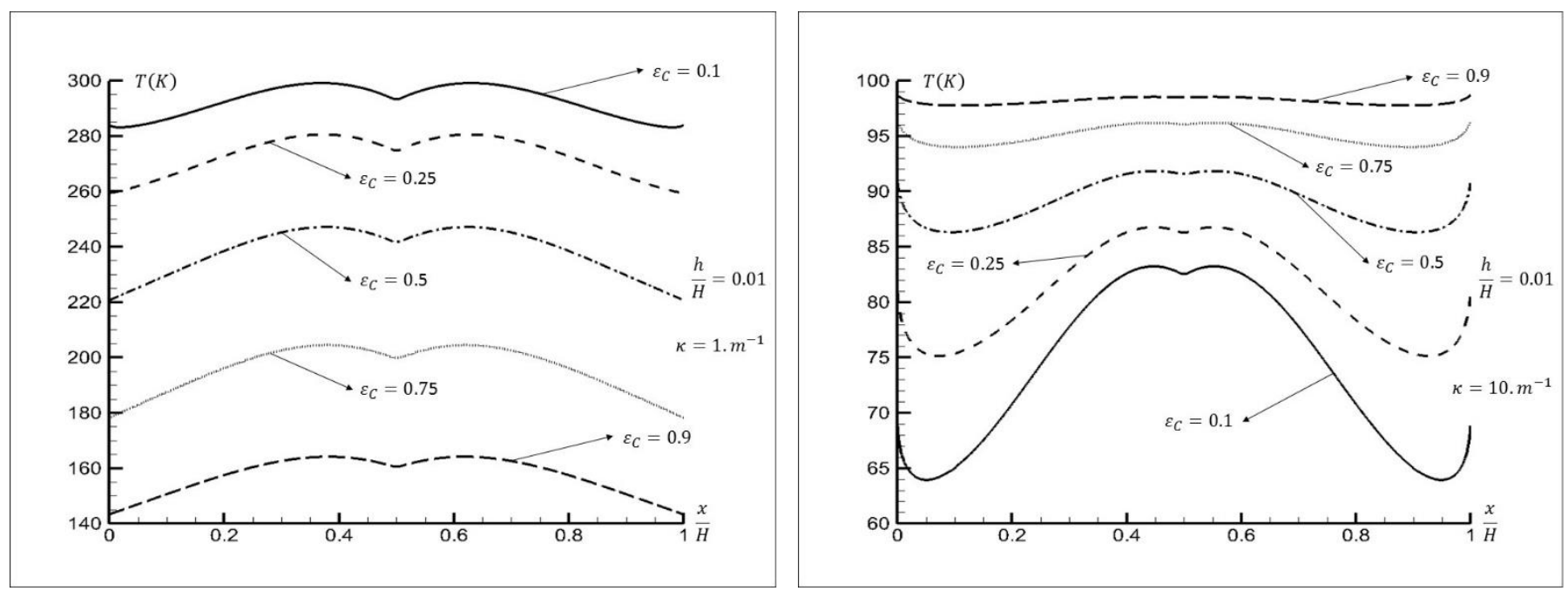

Figure 8: Cavity's radiosity temperature for various emissivities on the cavity's surfaces when $\frac{h}{H}=0.01$, for two absorption coefficients

One notes that increasing the emissivity of the cavity's surfaces doesn't dramatically change the morphologic structure of the radiosity temperatures at any absorption coefficient, but strongly modifies their amplitudes. A same trend can be observed for higher ratios $\frac{h}{H}$.

The determination of the cavity ( and/or obstacle) radiosity temperatures making no assumption on the surfaces emissivity behaviour, we look at the case of a sinusoidal varying emissivity on the four cavity's surfaces when the obstacle's surfaces are black, to precise the influence of the emissivity on the radiosity 
temperatures for a cold emitting and absorbing internal medium, with $T_{C}=1000 \mathrm{~K}$ and $T_{O}=100 \mathrm{~K}$. We examine the influence of the medium's absorption coefficient for different ratios $\frac{h}{H}$ when the cavity's surfaces emissivity variation is given by $\varepsilon_{C}=\frac{1}{10}\left[1-\sin \left(\frac{7 \pi x}{H}\right)\right]$, symmetric relatively to the surface's centre. The numerical results are described in Figs. 9a-d.

- $\quad$ Case $\frac{h}{H}=0.1:\left(\frac{h}{H}<\sqrt{5}-2\right)$
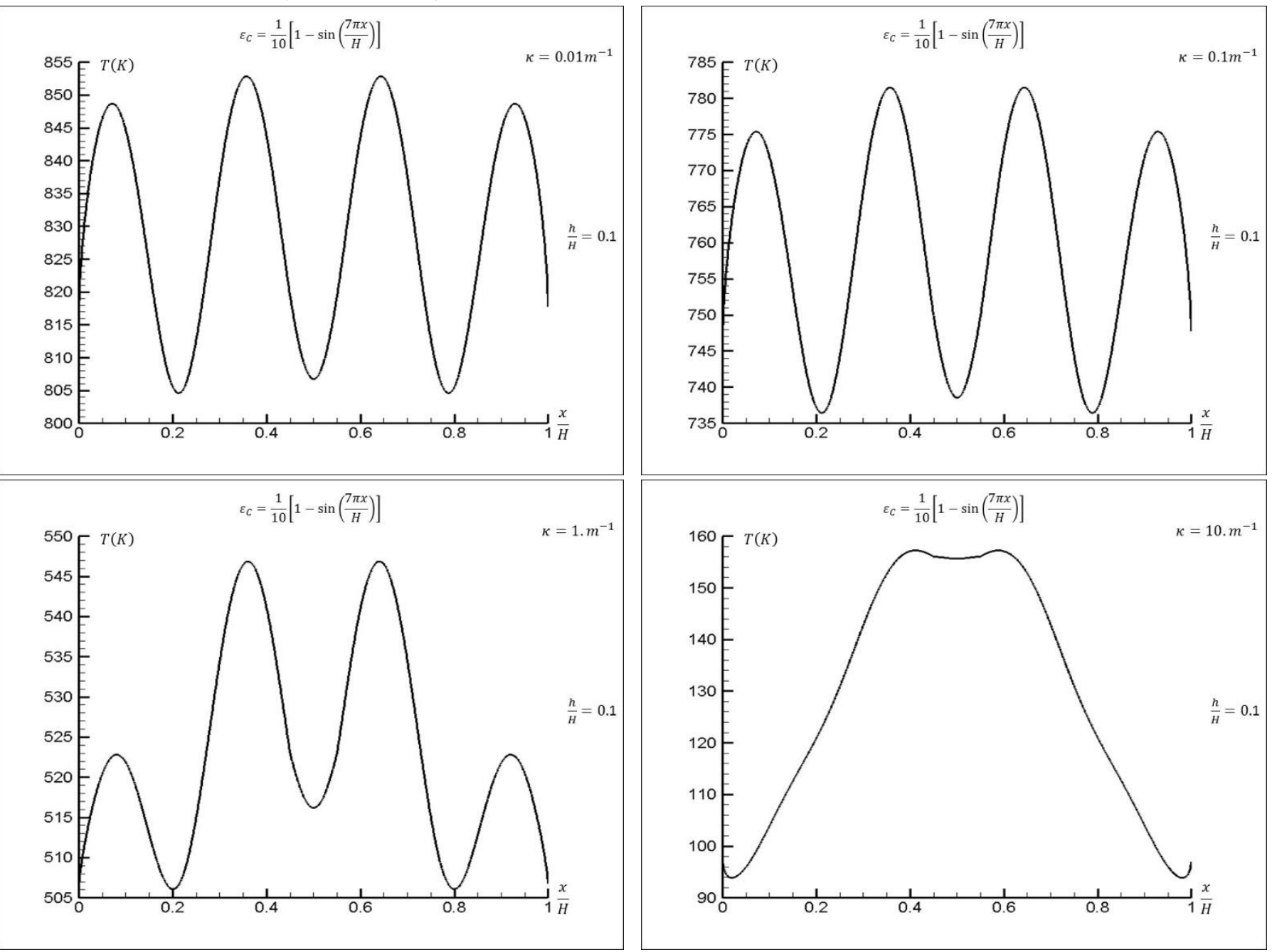

Figure 9a: Cavity's radiosity temperature for $\frac{h}{H}=0.1$ with $T_{C}=1000 \mathrm{~K}, T_{O}=100 \mathrm{~K}$ and $\varepsilon_{O}=1$ for different absorption coefficients

- $\quad$ Case $\frac{h}{H}=0.3:\left(\sqrt{5}-2<\frac{h}{H}<\frac{1}{3}\right)$ 

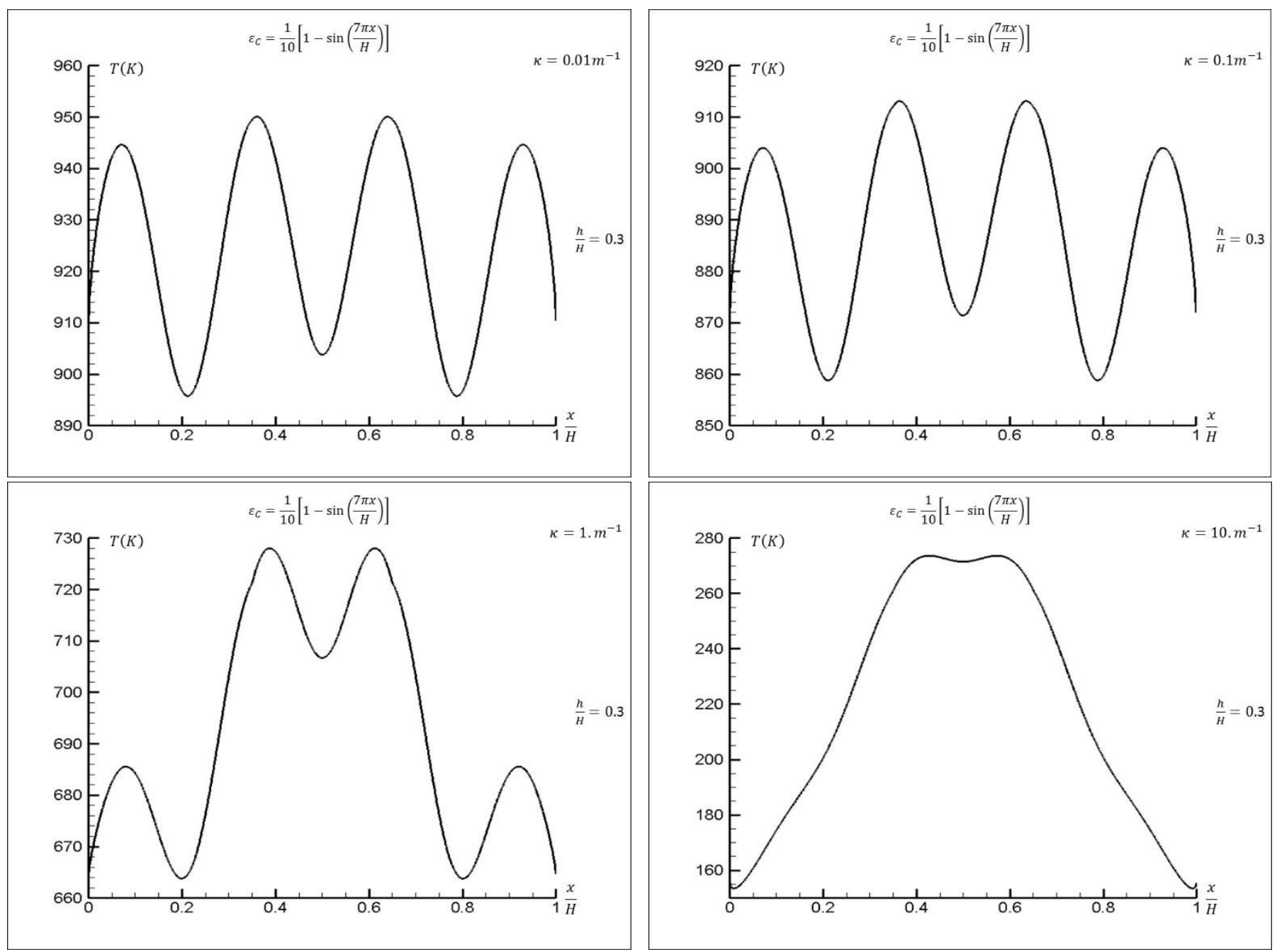

Figure 9b: Cavity's radiosity temperature for $\frac{h}{H}=0.3$ with $T_{C}=1000 \mathrm{~K}, T_{O}=100 \mathrm{~K}$ and $\varepsilon_{O}=1$ for different absorption coefficients

- $\quad$ Case $\frac{h}{H}=0.5:\left(\frac{h}{H}>\frac{1}{3}\right)$
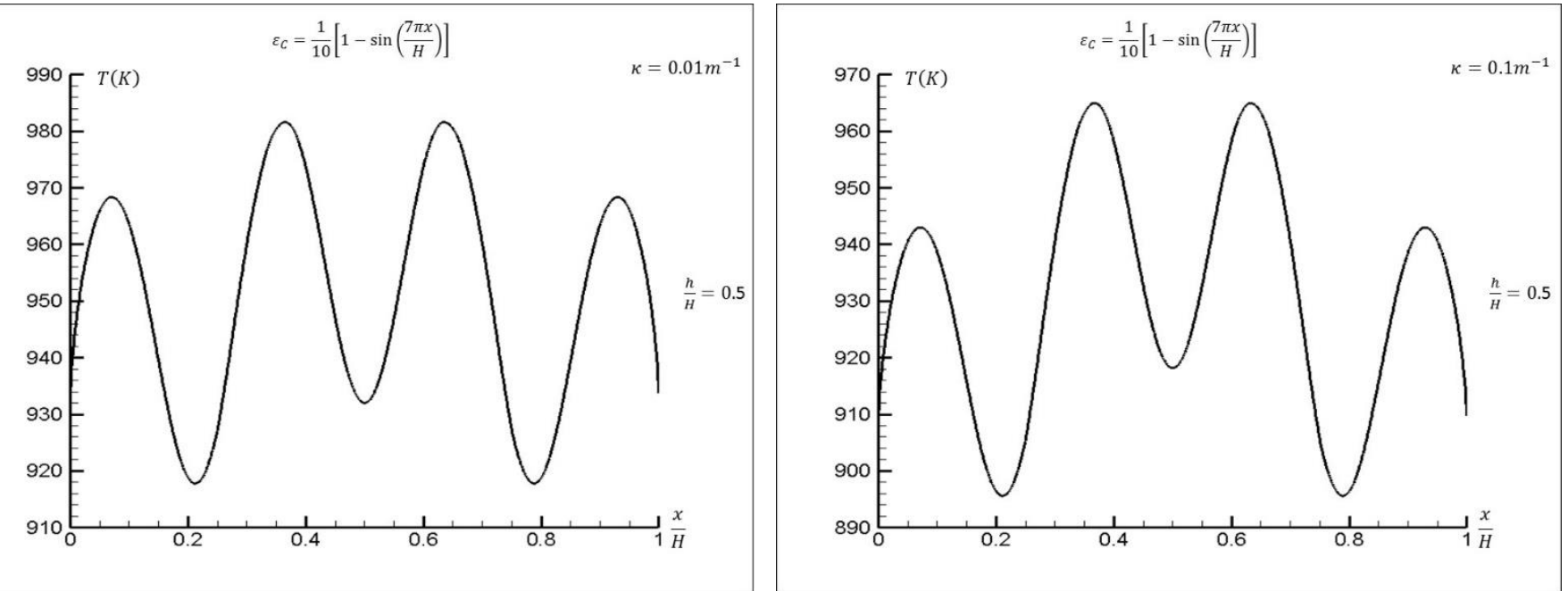

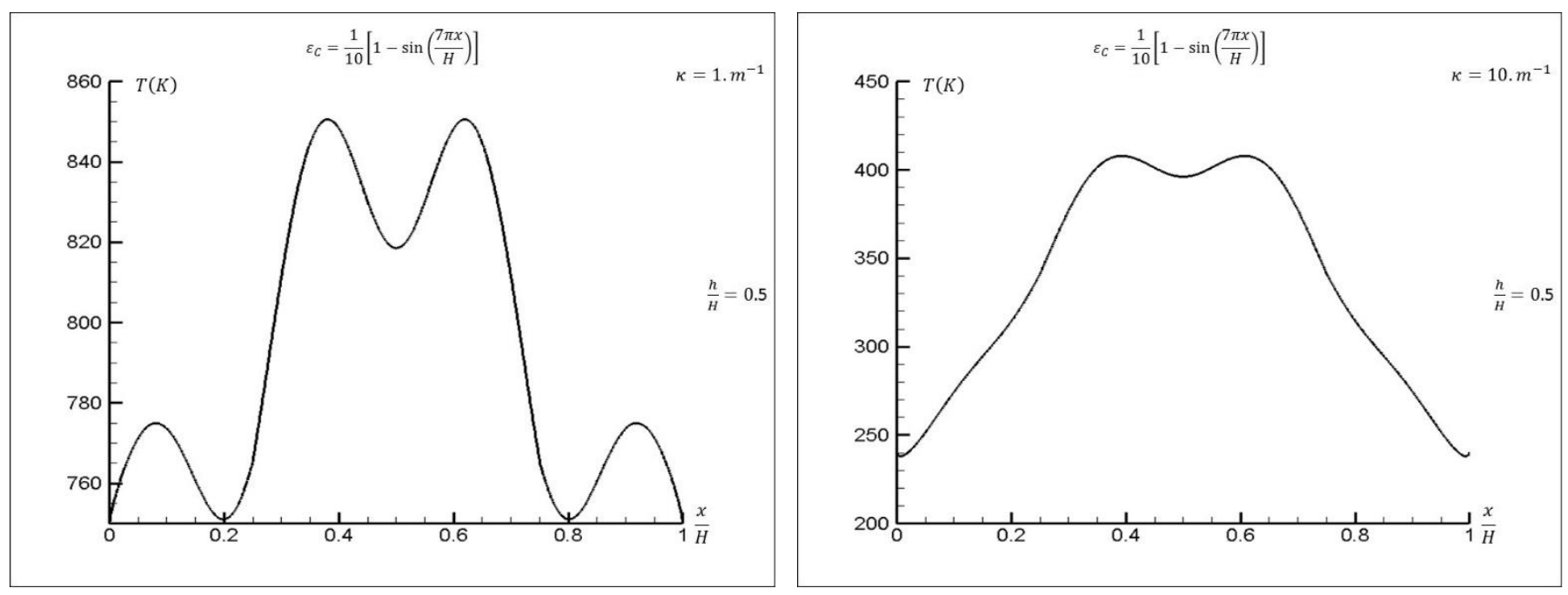

Figure 9c: Cavity's radiosity temperature for $\frac{h}{H}=0.5$ with $T_{C}=1000 \mathrm{~K}, T_{O}=100 \mathrm{~K}$ and $\varepsilon_{O}=1$ for different absorption coefficients

- $\quad$ Case $\frac{h}{H}=0.9:\left(\frac{h}{H}>\frac{1}{3}\right)$
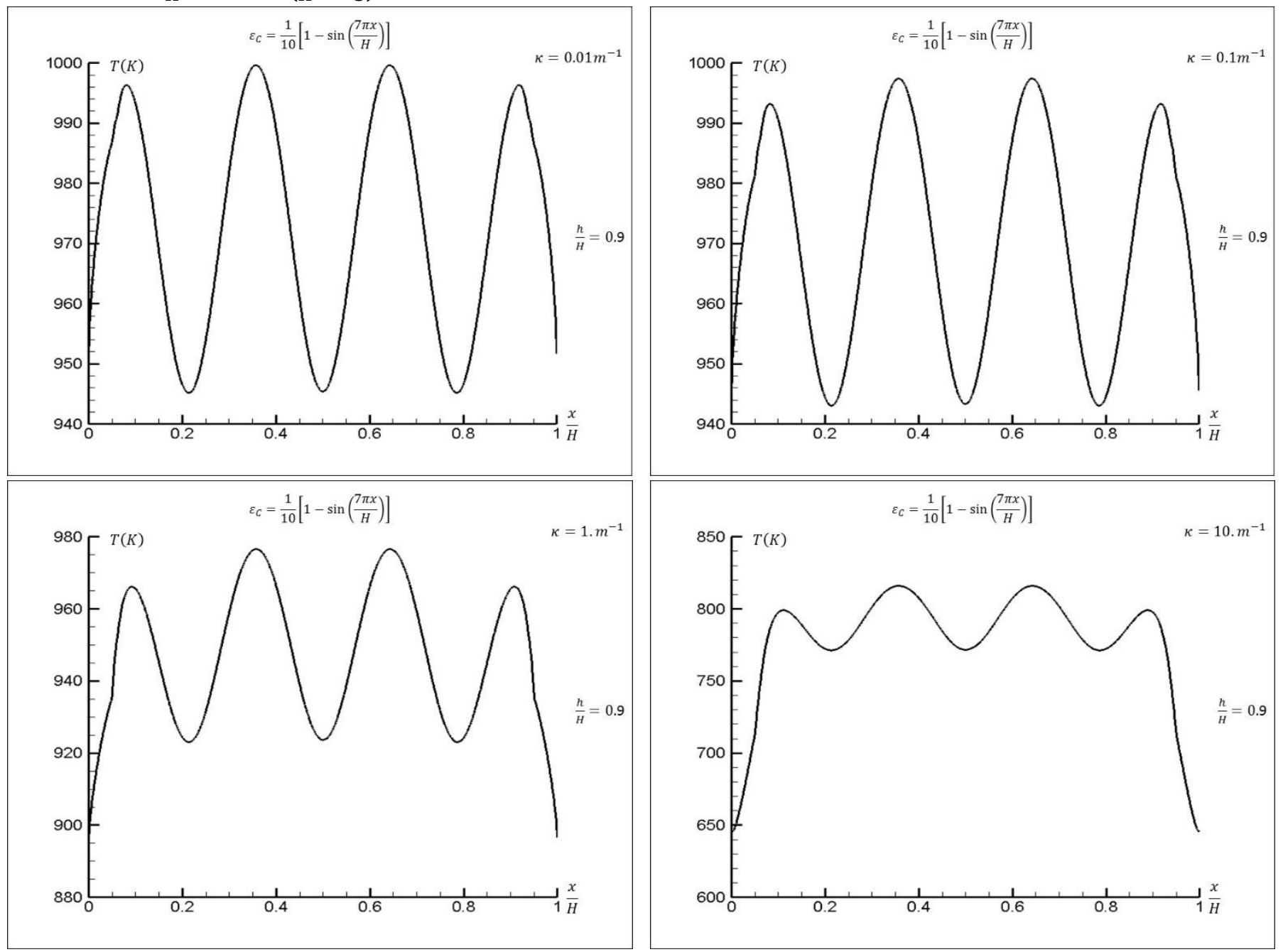

Figure 9d: Cavity's radiosity temperature for $\frac{h}{H}=0.9$ with $T_{C}=1000 \mathrm{~K}, T_{O}=100 \mathrm{~K}$ and $\varepsilon_{O}=1$ for different absorption coefficients

Note that the morphologic structure of the radiosity temperature is strongly modified by the spatial variation of the emissivity on the surface. One observes that for an emissivity perfectly symmetric relatively to the centre of the cavity, the radiosity temperatures are identical on the four cavity's surfaces. 
For a slightly modified emissivity $\varepsilon_{C}=\frac{1}{10}\left[1-\sin \left(\frac{2 \pi x}{H}\right)\right]$, the latter one is no longer symmetric relatively to the cavity's centre but antisymmetric, and the problem is no longer geometrically symmetric: this can be analized on the radiosity temperatures which are not symmetric and moreover different on the surfaces, as exemplified in Fig. 10 for a small ratio $\frac{h}{H}=0.1$, for four different absorption coefficients:
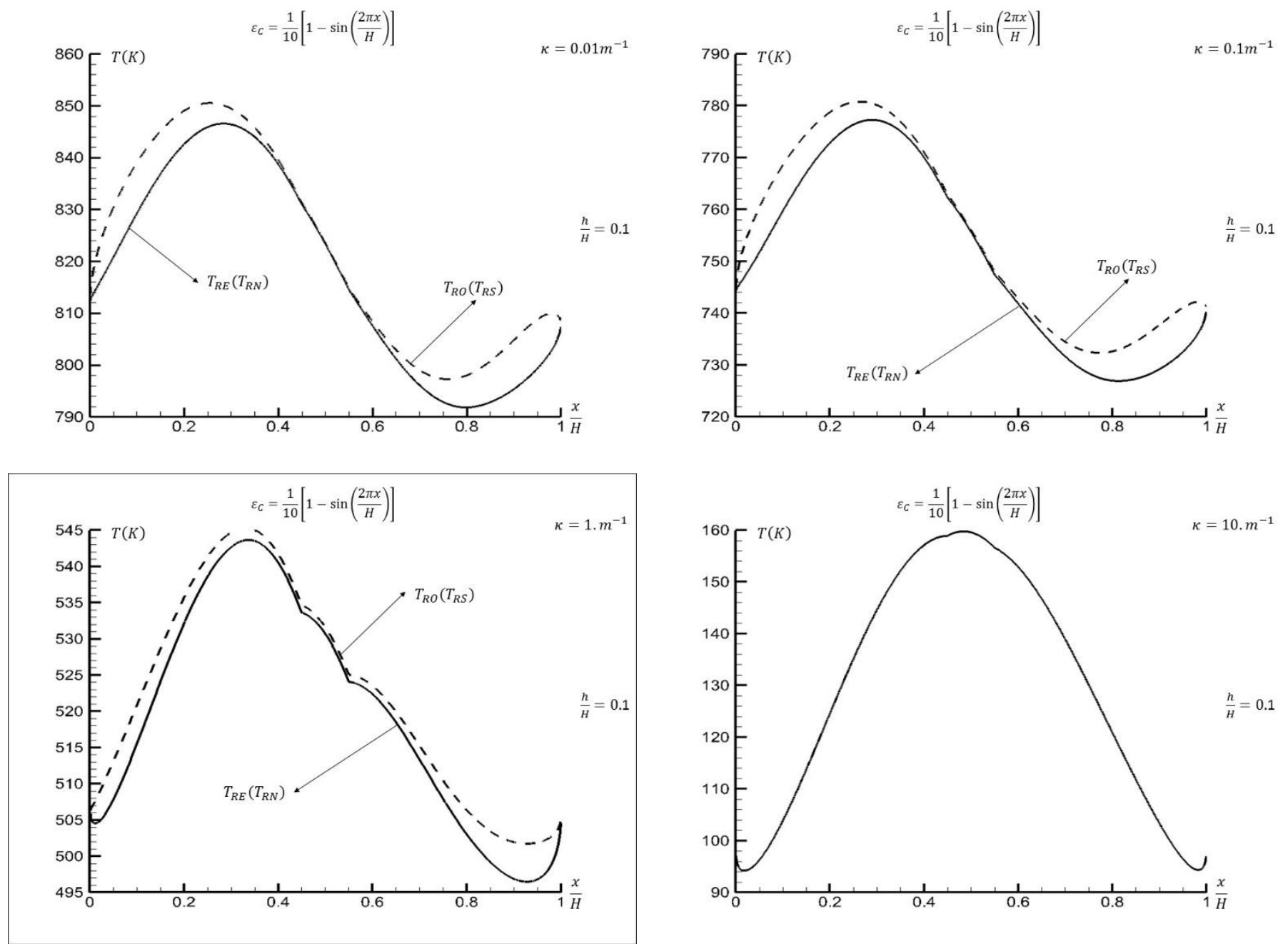

Figure 10: Cavity's radiosity temperature for $\frac{h}{H}=0.1$ and four different absorption coefficients

Taking now into account the ability of reflecting obstacle's surfaces, we examine the case of almost black cavity's surfaces with $\varepsilon_{C}=0.95$ with $T_{C}=1000 \mathrm{~K}$, and a much colder highly reflecting obstacle with $T_{O}=100 \mathrm{~K}$ and $\varepsilon_{O}=0.05$, for four different absorption coefficients. The results are reported in Fig. 11 for a ratio $\frac{h}{H}=0.5$, which insures to have a noticeable effect of the cavity's surfaces on the obstacle. 


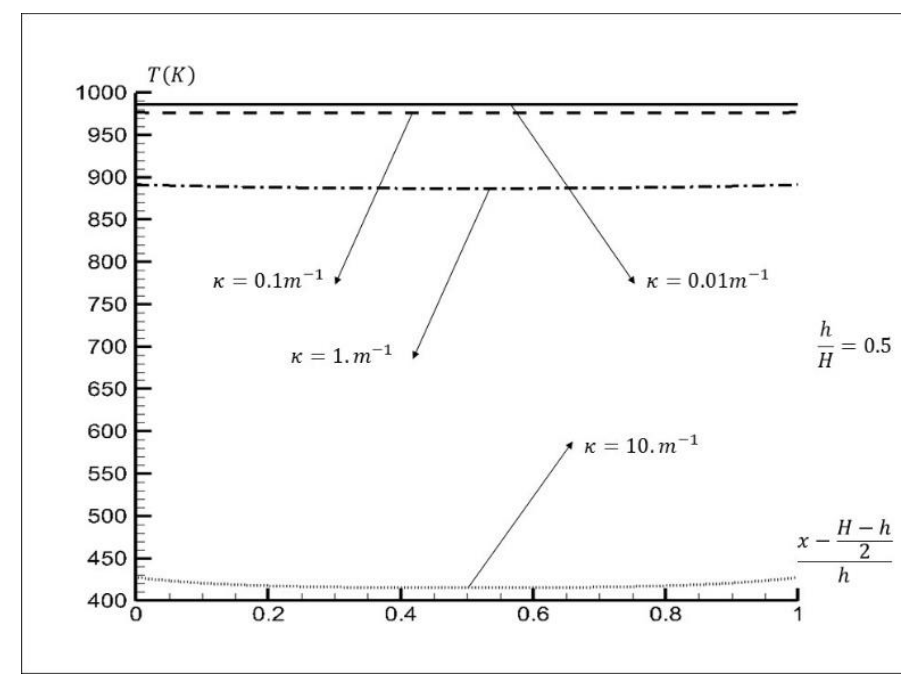

Figure 11: Obstacle's radiosity temperature for various absorption coefficients when $\frac{h}{H}=0.5$

As it can be seen in Fig. 11 the obstacle's radiosity temperature is almost constant on each whole surface for small absorption coefficients, which is more verified for small ratios $\frac{h}{H}$, while the radiosity temperature smoothly evolves when increasing the absorption coefficient. Is has been numerically observed that the obstacle's radiosity temperature spatial field is not mainly affected by the reflexion and imposed temperature conditions on the physical surfaces of the global device, but the mean magnitude is strongly depending on the different parameters.

If one furthermore takes into account the effect of the isothermal medium temperature $T_{m}$ on the radiosity temperatures, exemplified here in Fig. 12 for a cold $\left(T_{C}=100 \mathrm{~K}\right)$ highly reflecting cavity $\varepsilon_{C}=0.1$ and hot $\left(T_{O}=1000 \mathrm{~K}\right)$ almost black obstacle $\varepsilon_{O}=0.9$, we notice that it plays a significant influence on the cavity's radiosity temperature, especially for moderate to high absorption coefficients, both on the structure field and magnitude. For a small absorption however, the influence of the medium's temperature is less important.
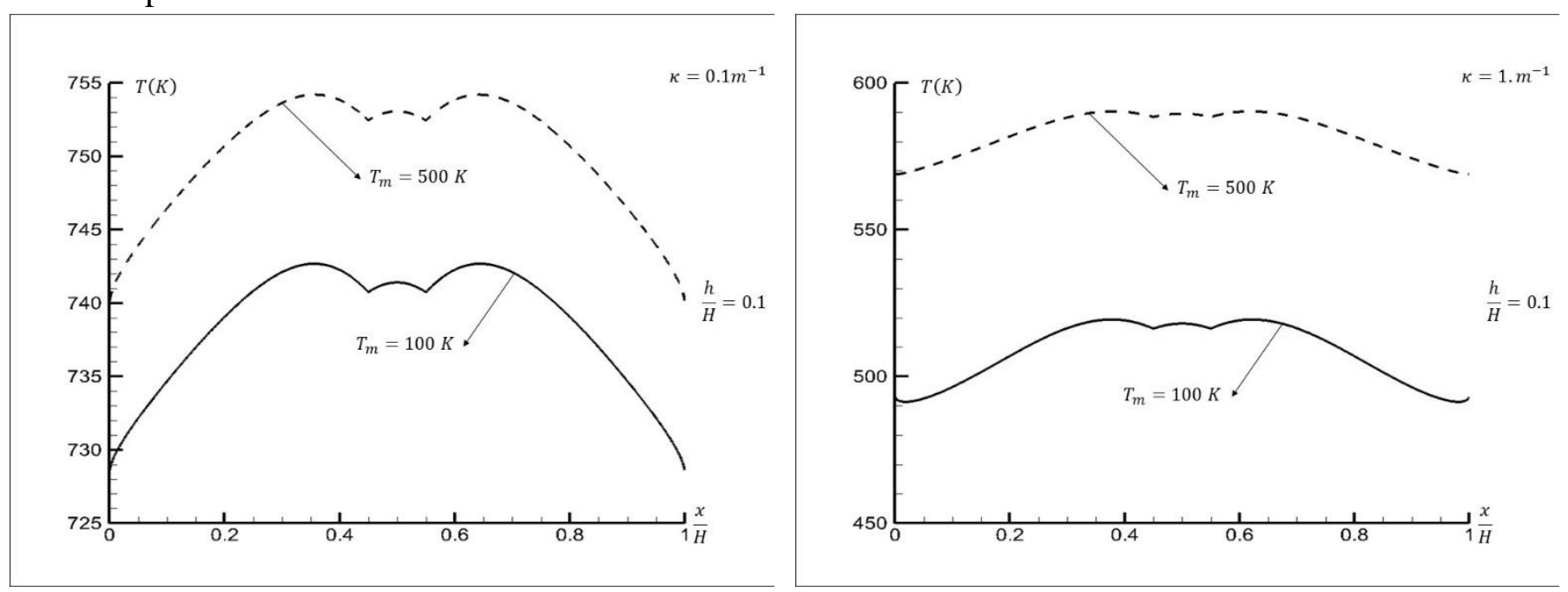


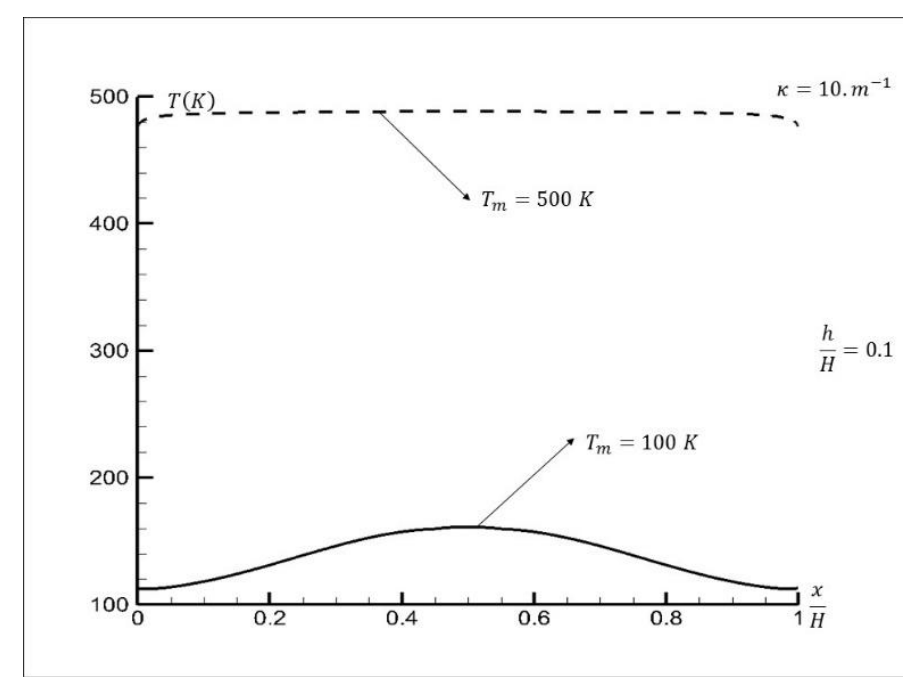

Figure 12: Cavity's radiosity temperature for various absorption coefficients when $\frac{h}{H}=0.1$ and for two isothermal media of different temperature

\section{IV.2.1. Non-isothermal semi-transparent media:}

The case of a non-isothermal internal absorbing-emitting medium has also been examined. We present hereafter some simulation cases of cavity's radiosity temperatures when the emitting-absorbing medium is non-isothermal, the central obstacle's surfaces still being black.

In all examined following cases, the boundary conditions on the cavity and obstacle's surfaces are kept to be $T_{C}=1000 \mathrm{~K}, T_{O}=100 \mathrm{~K}, \varepsilon_{C}=0.1$ and $\varepsilon_{O}=1$ and the internal medium is subjected to an imposed sinusoidal spatially varying temperature field with $T_{0}=500 \mathrm{~K}$. The results are described for different $\operatorname{ratios} \frac{h}{H}$.

In the first case exemplified in Figs 13a-c, the temperature field of the absorbing-emitting medium is symmetric relatively to the cavity's centre with a sinusoidal variation given by

$T(x, y)=T_{0}\left\{1+\frac{1}{2} \cos \left[\frac{4 \pi \sqrt{\left(\frac{H}{2}-x\right)^{2}+\left(\frac{H}{2}-y\right)^{2}}}{H}\right]\right\}$

\section{IV.2.1a. Sinusoidal completely symmetric internal temperature field:}

- $\quad$ Case $\frac{h}{H}=0.1$ :
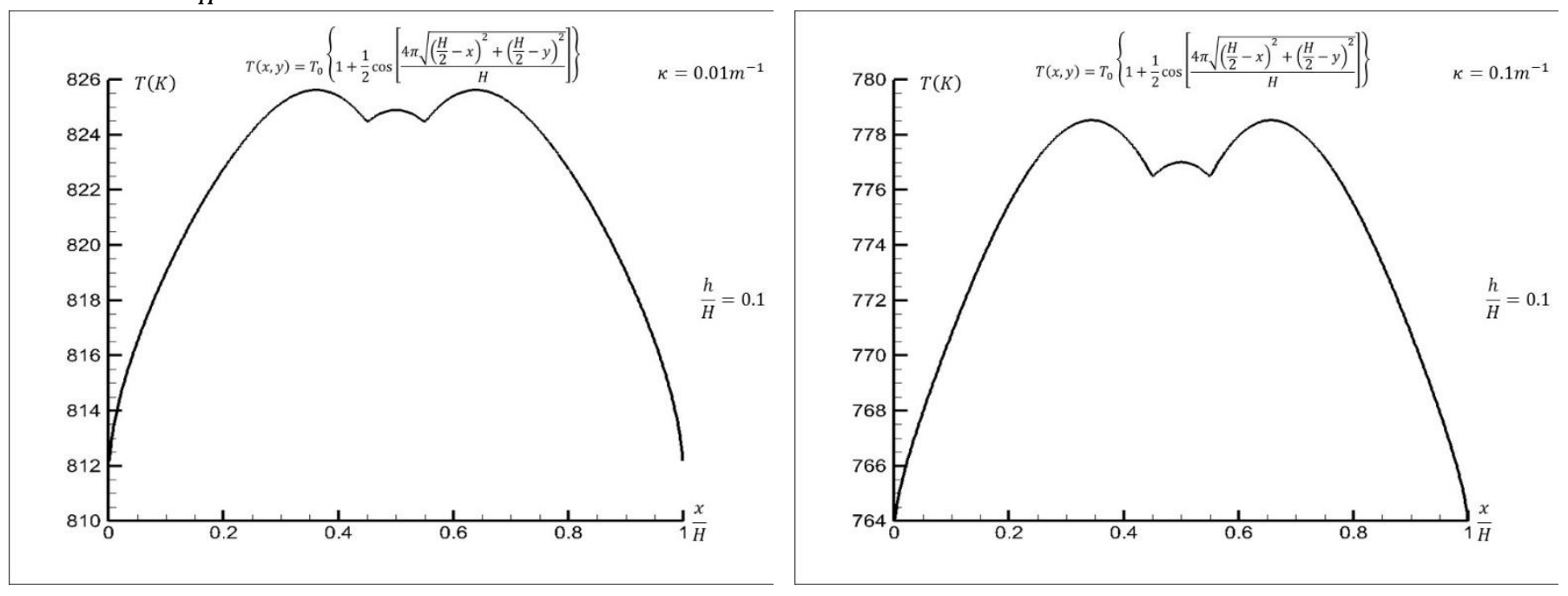

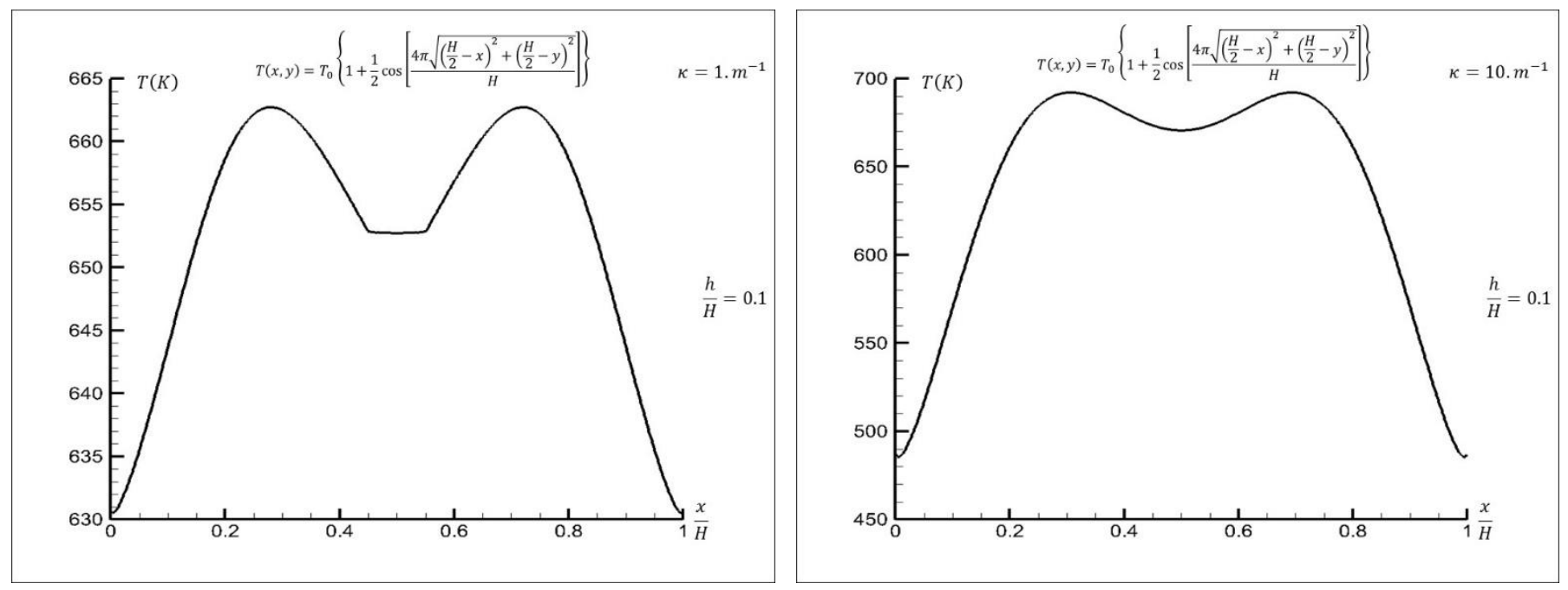

- $\quad$ Case $\frac{h}{H}=0.3$ :
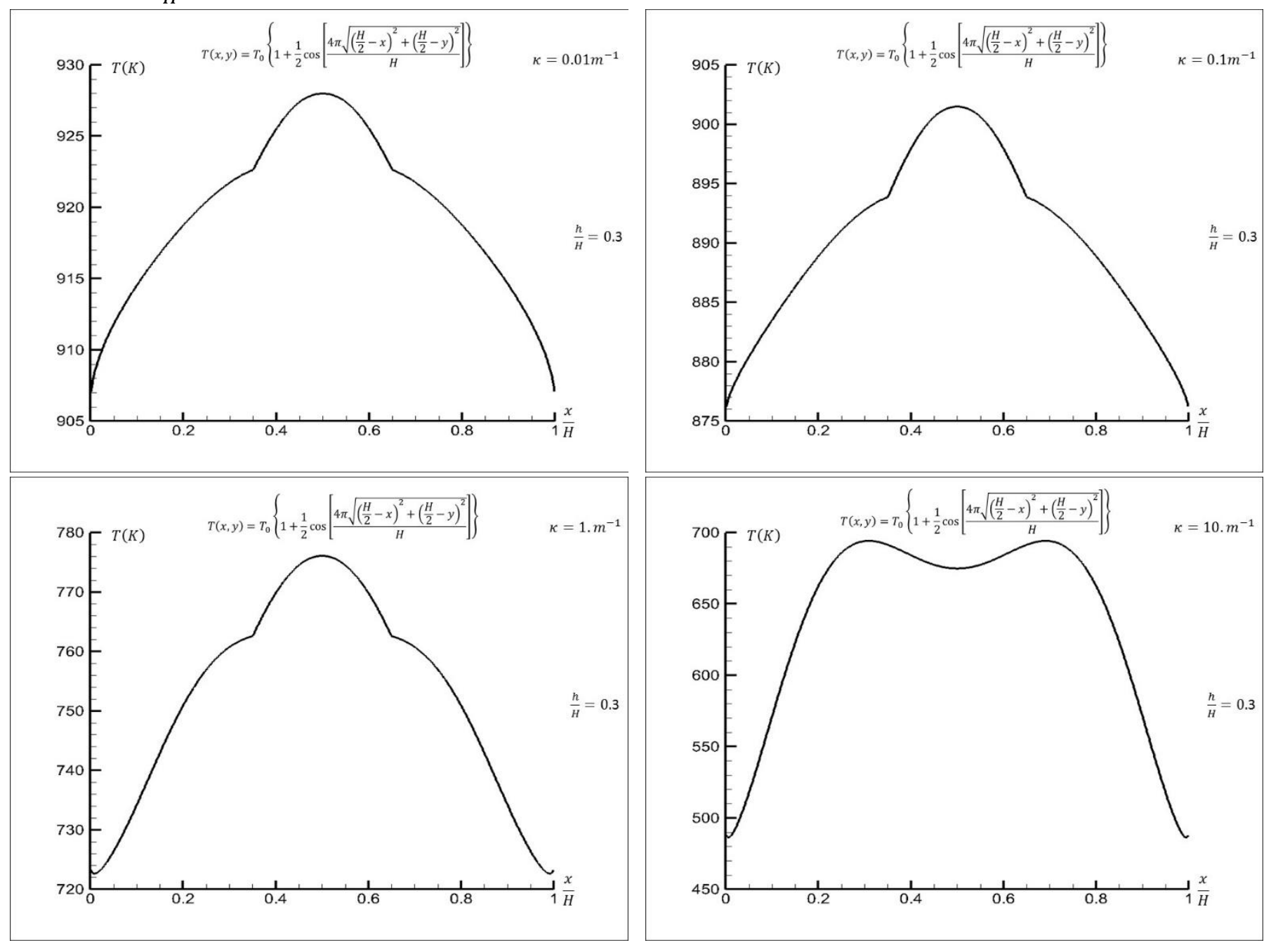

- $\quad$ Case $\frac{h}{H}=0.5$ : 

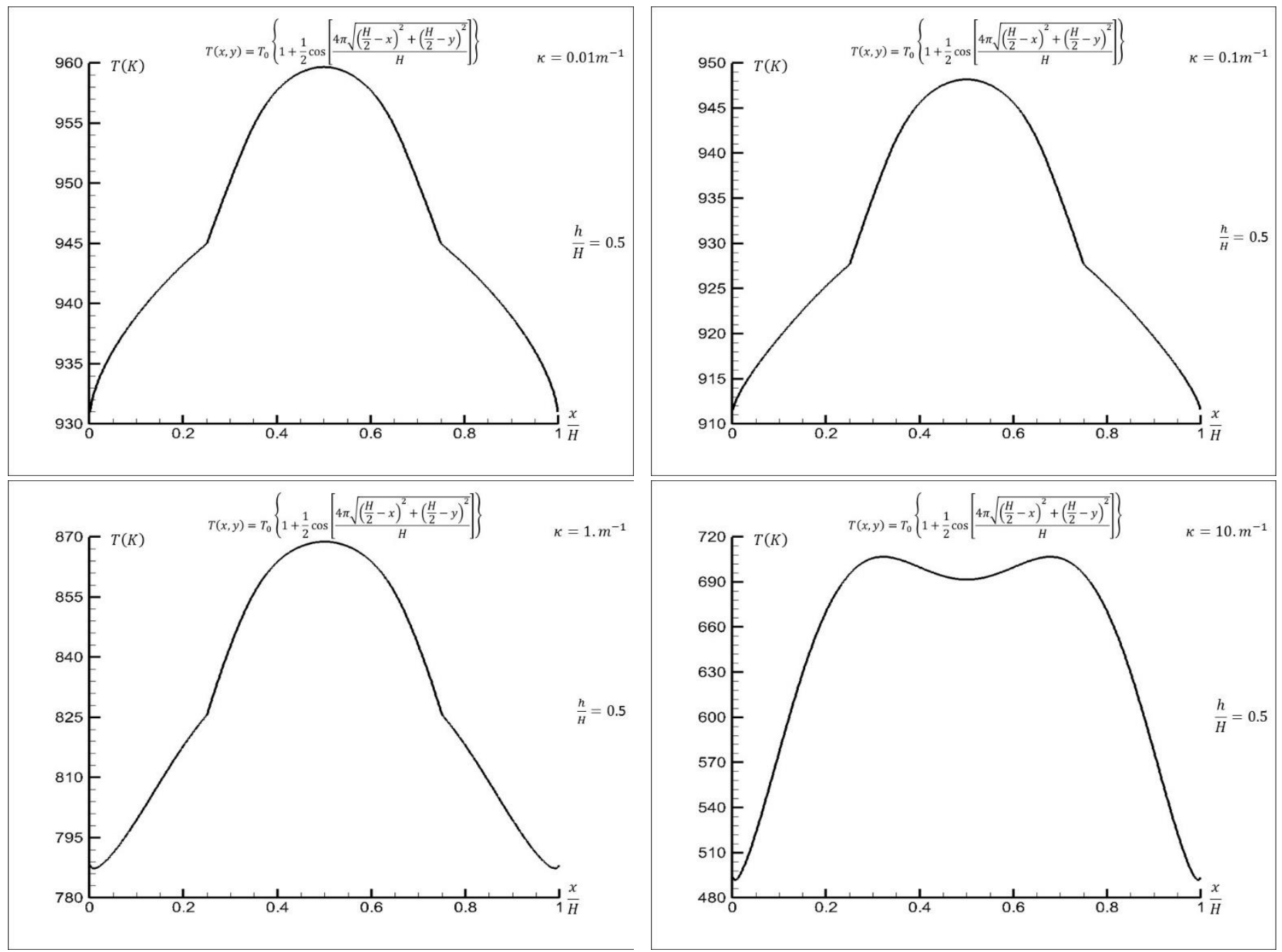

Figure 13a-c: Cavity's surface radiosity eastern and western temperatures for a completely symmetric sinusoidal temperature field with $T_{0}=500 \mathrm{~K}$ for three characteristic ratios $\frac{h}{H}$ and four different absorption coefficients

One observes that when the temperature field is symmetric relatively to the cavity's center, the four radiosity temperatures on the cavity surfaces are equal, with a shape highly similar to the one observed in the case of an isothermal medium, except for the case of media with relatively high absorption coefficients for which the influence of the non-isothermal field is preponderant.

\section{IV.2.1b. Sinusoidal completely non symmetric internal temperature field:}

In the second case illustrated in Figs 14a-c, the temperature field of the absorbing-emitting medium is completely non-symmetric relatively to the cavity's centre with a sinusoidal analogous variation given by $T(x, y)=T_{0}\left\{1+\frac{1}{2} \cos \left[\frac{4 \pi \sqrt{\left(\frac{h}{2}-x\right)^{2}+y^{2}}}{H}\right]\right\}$

- $\quad$ Case $\frac{h}{H}=0.1$ : 

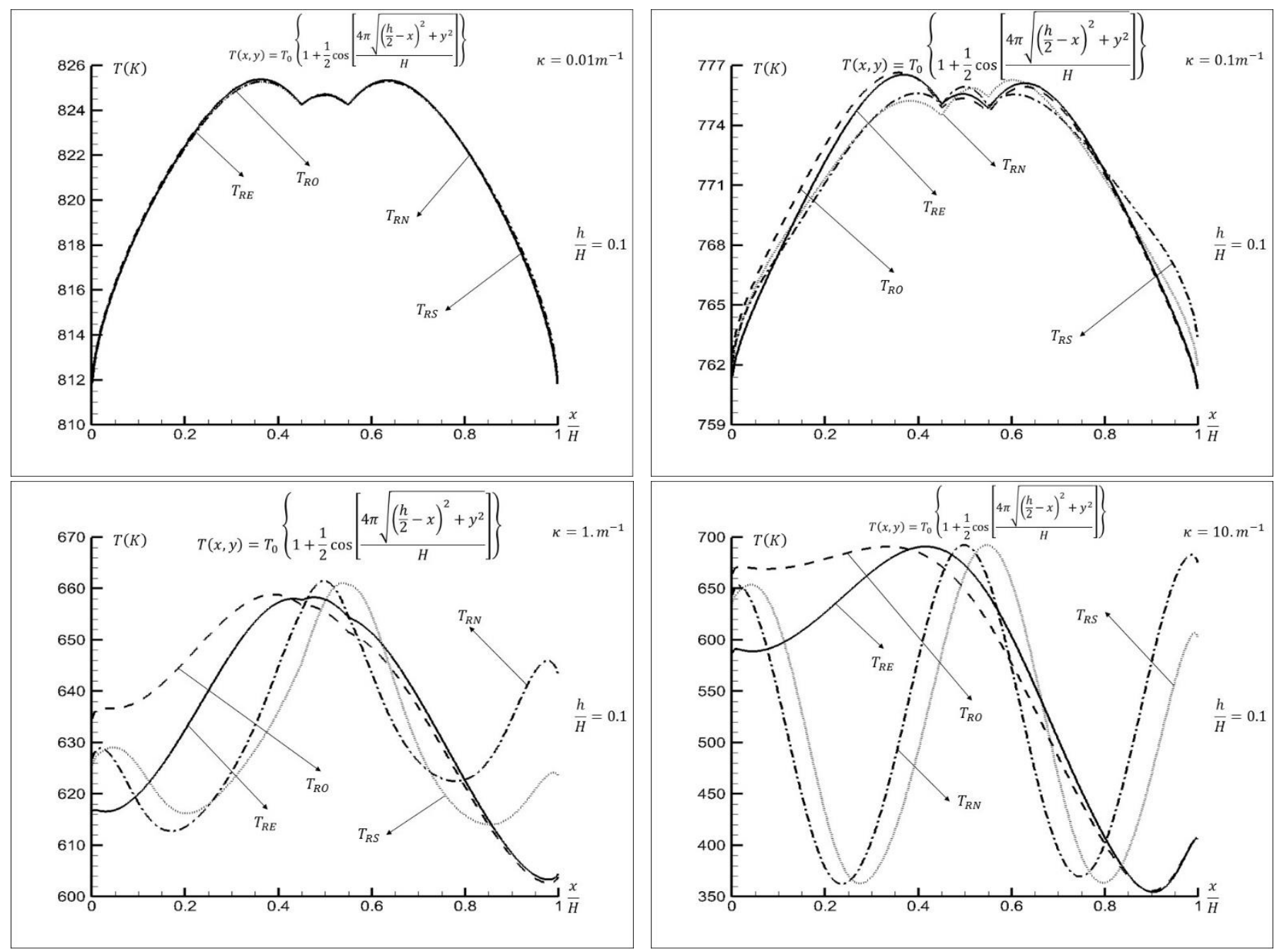

- $\quad$ Case $\frac{h}{H}=0.3$ :
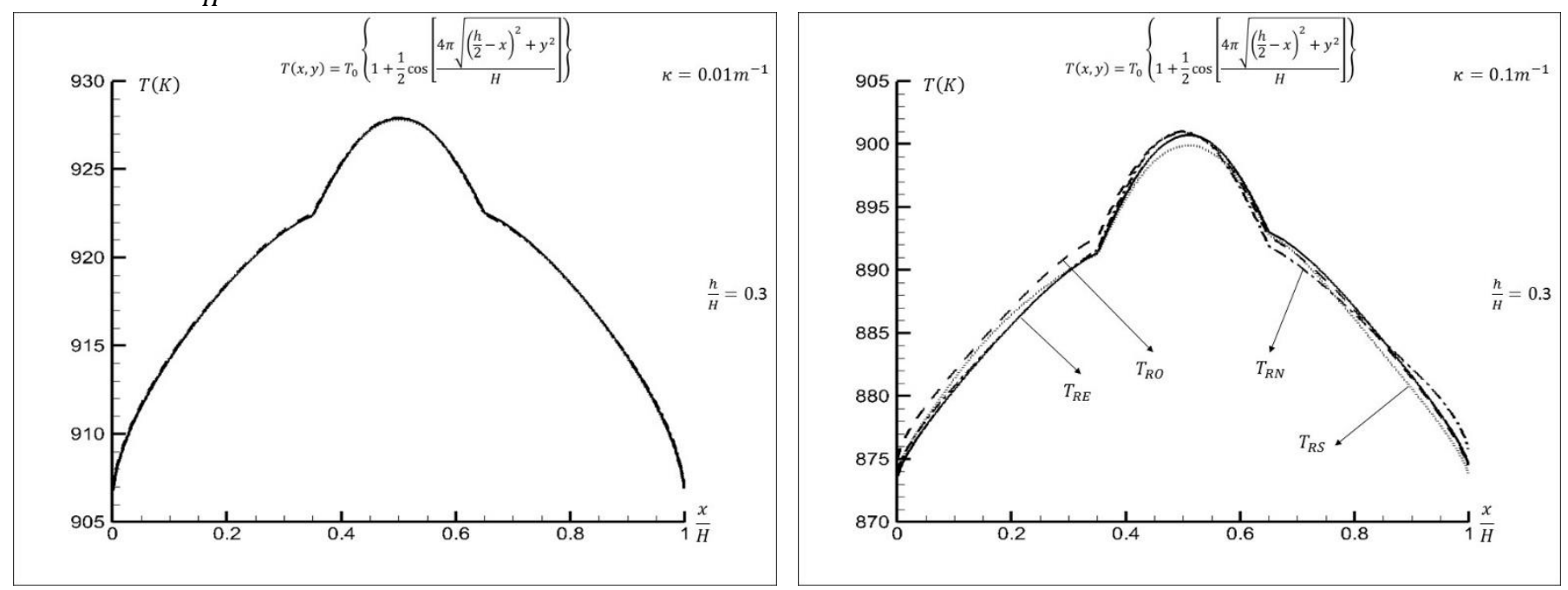

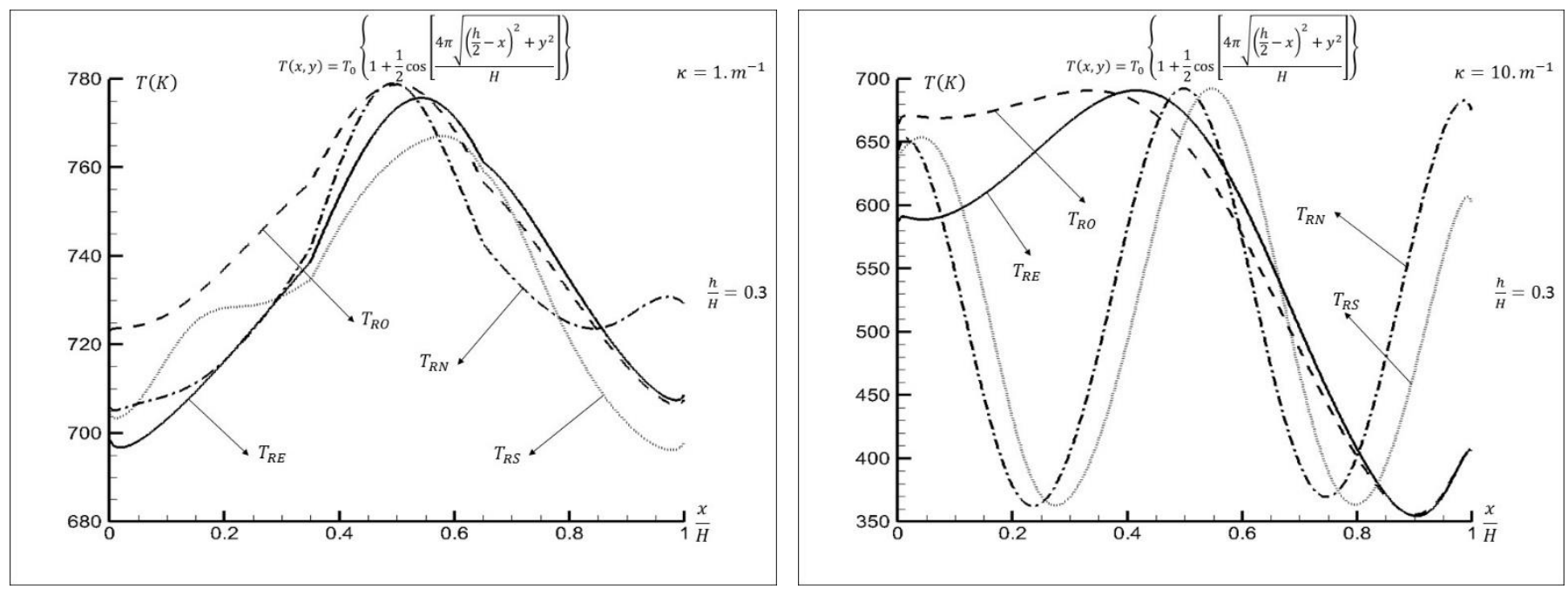

- $\quad$ Case $\frac{h}{H}=0.5$ :
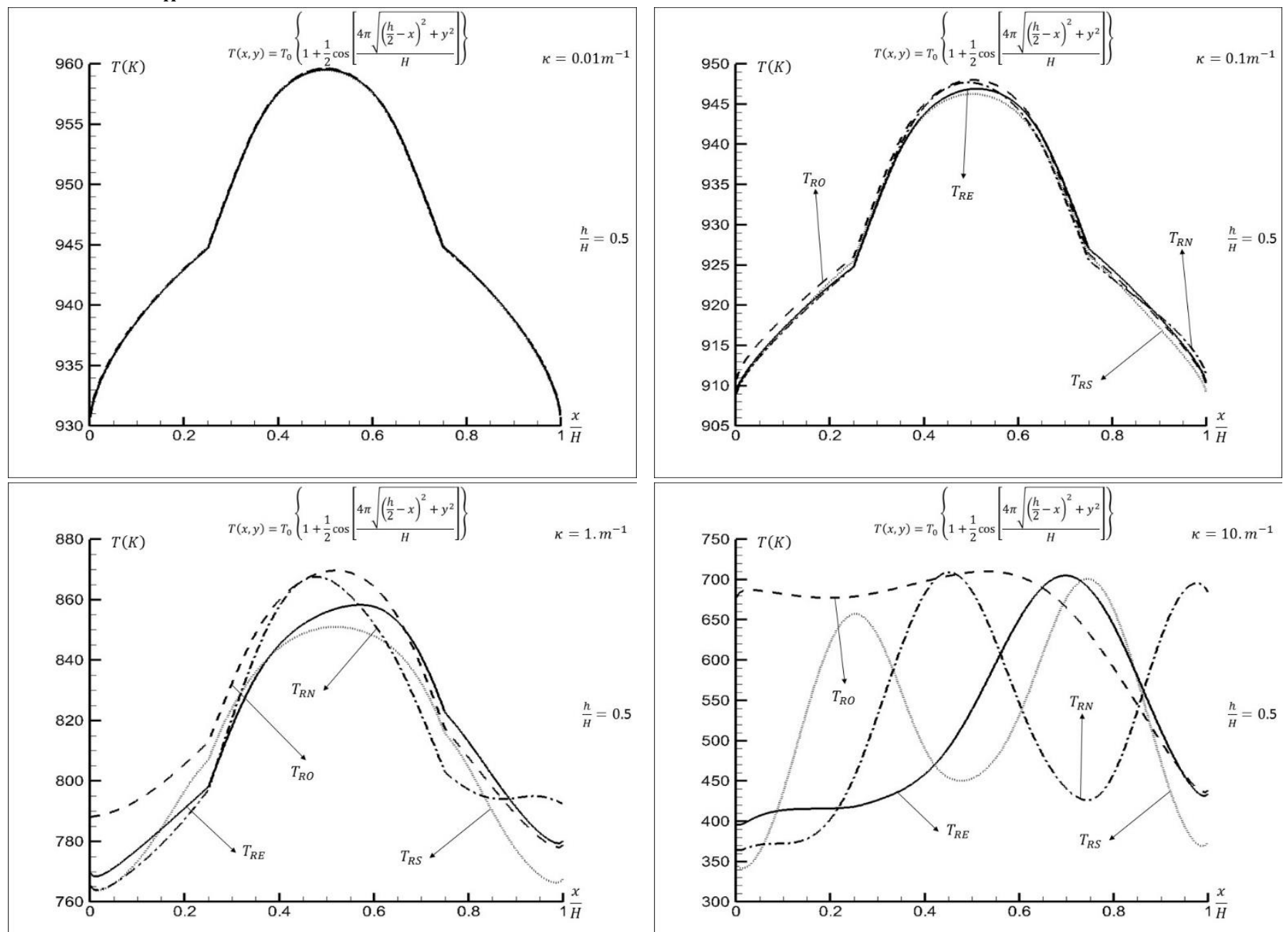

Figure 14a: Cavity's surface radiosity eastern and western temperatures for a non-symmetric sinusoidal temperature field with $T_{0}=500 \mathrm{~K}$ for three characteristic ratios $\frac{h}{H}$ and four different absorption coefficients

When the temperature field of the internal medium is no longer symmetric relatively to the center we note that logically the radiosity temperatures are different on the four surfaces of the cavity, especially for mean optical depths around or greater than one. For small absorption coefficients however, the radiosity temperatures are very similar in each case on the four surfaces, which suggest that the radiosity 
temperatures are not strongly affected by the temperature field of the internal medium: this let suspect that when determining the radiative field inside the medium in presence of reflecting surfaces, the radiative coupling between the internal medium and reflecting surfaces is low if the absorption coefficient of the internal medium is small, while the coupling may become strong for moderate to highly absorbing media.

To terminate this numerical section, we finally present some miscellaneous cases combining spatial varying emissivities and internal temperature field, in a completely symmetric case and a mixed symmetric/antisymmetric device, hereafter illustrated in Figs. 15-16 for a given absorption coefficient $\kappa=1 . m^{-1}$

In the first case, the emissivity is equal on the four surfaces of the cavity, with a sinusoidal symmetric spatial evolution relatively to the centre of the surfaces. Combining to this emissivity behaviour a perfectly symmetric sinusoidal evolution relatively to the cavity's centre of the internal temperature field, leads to a symmetric variation of the radiosity temperature on the surface, for each surface, mainly depending on the ratio $\frac{h}{H}$ for a given absorption coefficient and prescribed set of surfaces imposed temperatures, as it can be observed in Fig. 15
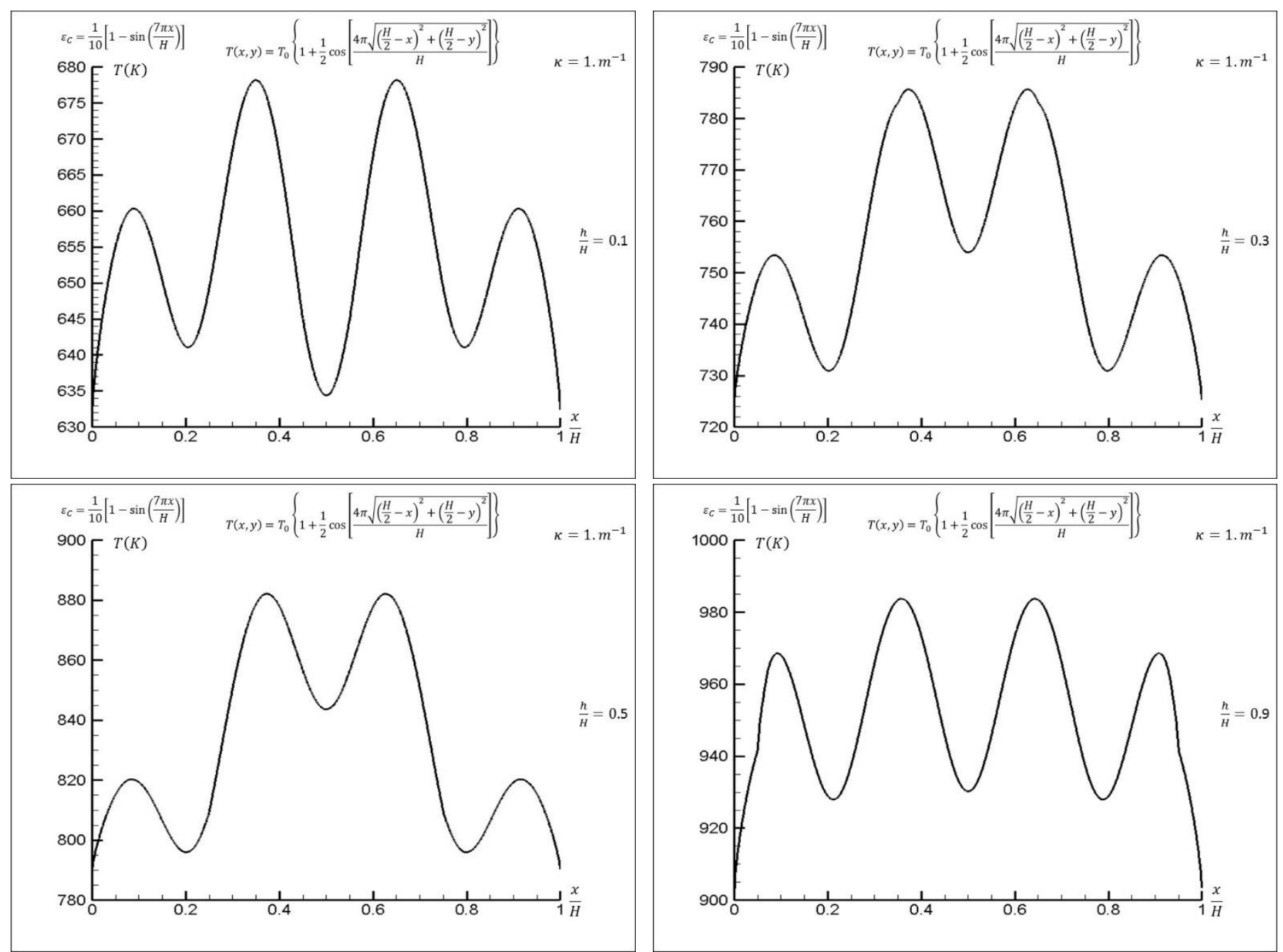

Figure 15: Symmetric sinusoidal variations of the cavity's surfaces emissivity and internal temperature field with $T_{0}=500 \mathrm{~K}$ for three characteristic ratios $\frac{h}{H}$ for a moderate absorption coefficient

In the second case, the temperature of the internal medium keeps the same symmetric sinusoidal variation and the cavity's four surfaces emissivities are such that $\varepsilon_{E}(y)=\varepsilon_{O}(y)$ and $\varepsilon_{N}(x)=\varepsilon_{S}(x)$, with 

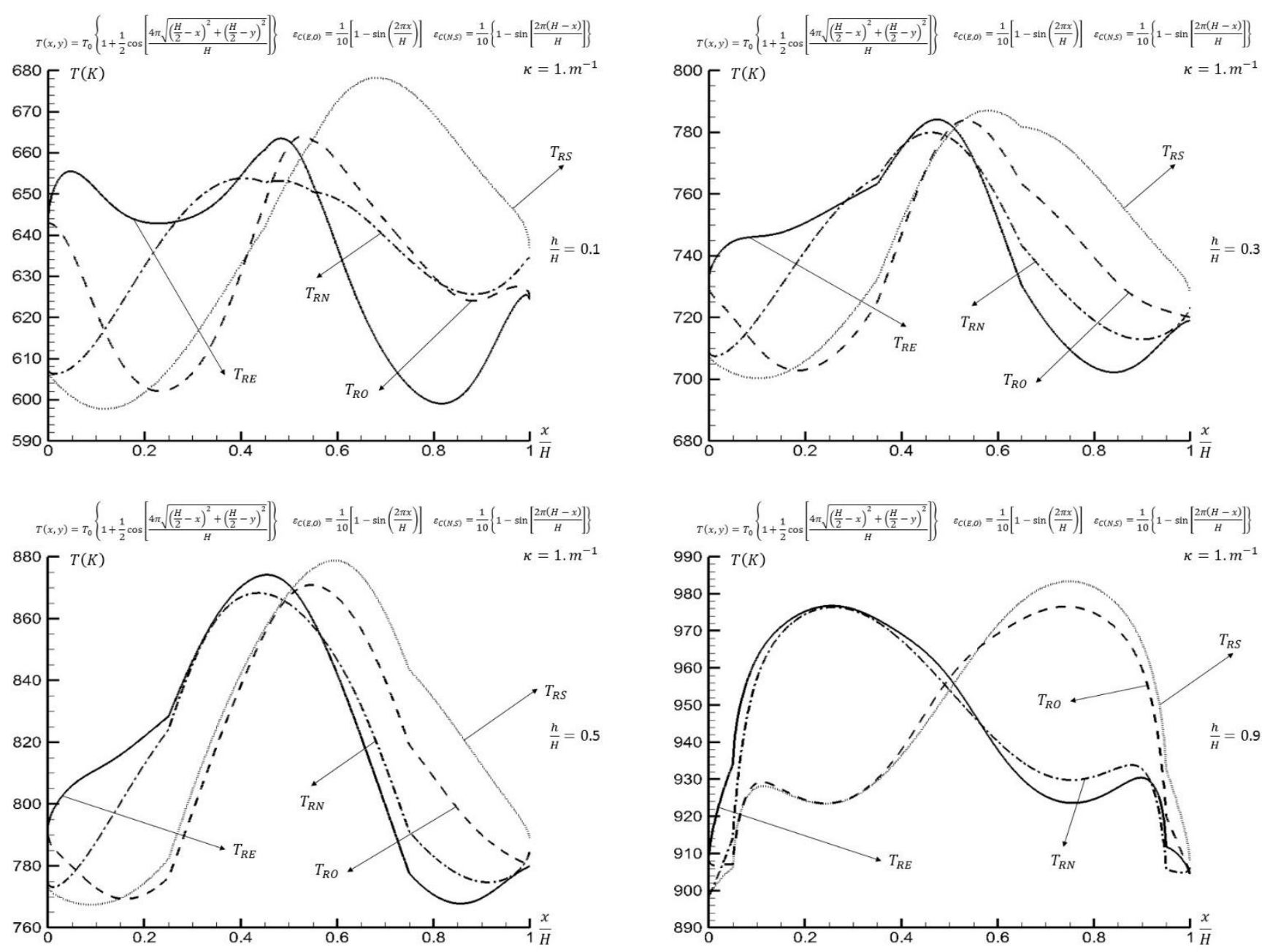

Figure 16: Antisymmetric sinusoidal variation of the cavity's surfaces emissivity and symmetric internal temperature field with $T_{0}=500 \mathrm{~K}$ for three characteristic ratios $\frac{h}{H}$ for a moderate absorption coefficient

The latter figure suggests that the internal radiative field is strongly depending on the reflection conditions on the surfaces of the device, an identical conclusion being done in the case of obstacle's reflecting surfaces, which let appear singular radiative behaviours in media at radiative equilibrium or in situation of radiation-conduction coupling.

\section{V - CONCLUDING REMARKS}

In this paper we completely described the exact analytical expressions of the radiosity temperatures on the diffusely reflecting surfaces of a cavity containing a centred opaque obstacle with reflecting surfaces, surrounded by a non-isothermal absorbing-emitting semi-transparent medium. We carefully detailed all the possible geometric configurations arising on each surface by putting in evidence the critical rule of the parameter $\frac{h}{H}$ already pointed out in [24], and consequently determined the associated expressions in each case. The lengths ratio appears to be a major parameter in the resulting radiative behaviour on the cavity's surfaces, which is also strongly depending on the internal medium's absorption coefficient so as the imposed surfaces temperatures and emissivities. The numerical results show that in some particular conditions of small absorptions and relative imposed surfaces temperatures magnitudes, the influence of the obstacle remains highly sensible even for extremely small obstacles 
comparatively to the cavity's surface size, which may have a strong incidence to the determination of the radiative field in the case of radiative equilibrium. It has also been pointed out that the space variation of the emissivity on the surfaces plays a major rule on the radiosity temperatures. The influence of the medium's temperature has also been examined, which may let suspect a strong radiative coupling between the cavity and obstacle surfaces and the internal medium in situation of radiative equilibrium or thermal coupling with conduction for moderately absorbing media. The determination of the incident radiation and radiative flux field in reflecting cavities with a reflecting obstacle is now under investigation before examining the case of radiative equilibrium and conductive-radiative coupling in the full device.

ANNEX 1: obstacle's radiosity temperatures for the western, norther and southern surfaces * Western obstacle's surface radiosity temperature:

$$
\begin{aligned}
\frac{\pi\left[T_{R \sigma_{O}}^{4}\left(\hat{y}_{k}\right)-\varepsilon_{\sigma_{O}} T_{\sigma_{O}}^{4}\right]}{2 \rho_{\sigma_{O}}} & \\
& =\int_{\varphi=0}^{\xi_{E}^{+}} T_{R O}^{4}\left(\tau_{k}^{+}+\tau_{0} \tan \varphi\right) K i_{3}\left(\frac{\tau_{0}}{\cos \varphi}\right) \cos \varphi d \varphi \\
& +\int_{\varphi=0}^{\xi_{E}^{-}} T_{R O}^{4}\left(\tau_{k}^{+}-\tau_{0} \tan \varphi\right) K i_{3}\left(\frac{\tau_{0}}{\cos \varphi}\right) \cos \varphi d \varphi \\
& +\int_{\varphi=0}^{\xi_{N}^{+}} T_{R N}^{4}\left(\tau_{0}-\tau_{k}^{-} \tan \varphi\right) K i_{3}\left(\frac{\tau_{k}^{-}}{\cos \varphi}\right) \sin \varphi d \varphi \\
& +\int_{\varphi=0}^{\xi_{S}^{+}} T_{R S}^{4}\left(\tau_{0}-\tau_{k}^{+} \tan \varphi\right) K i_{3}\left(\frac{\tau_{k}^{+}}{\cos \varphi}\right) \sin \varphi d \varphi+\kappa \aleph_{\sigma_{O}}
\end{aligned}
$$

where the internal contribution $\aleph_{\sigma_{O}}$ keeps the expression of Eq. (14) for isothermal media. When the medium is not isothermal, the internal contribution follows the same geometric substitution $\hat{x} \leftrightarrow H-\hat{x}$ and whence writes:

$$
\begin{aligned}
\kappa \aleph_{\sigma_{O}}=\int_{\tau_{u}^{+}=0}^{\tau_{0}} & \left\{\int_{\varphi=0}^{\xi_{E}^{+}} T^{4}\left(\tau_{0}-\hat{u}, \hat{y}_{k}+\hat{u} \tan \varphi\right) K i_{2}\left(\frac{\tau_{u}^{+}}{\cos \varphi}\right) d \varphi\right. \\
& \left.+\int_{\varphi=0}^{\xi_{E}^{-}} T^{4}\left(\tau_{0}-\hat{u}, \hat{y}_{k}-\hat{u} \tan \varphi\right) K i_{2}\left(\frac{\tau_{u}^{+}}{\cos \varphi}\right) d \varphi\right\} d \tau_{u}^{+} \\
& +\int_{\tau_{u}^{+}=0}^{\tau_{k}^{-}} \int_{\varphi=0}^{\xi_{N}^{+}} T^{4}\left(\tau_{0}-\hat{u} \tan \varphi, \hat{y}_{k}+\hat{u}\right) K i_{2}\left(\frac{\tau_{u}^{+}}{\cos \varphi}\right) \tan \varphi d \varphi d \tau_{u}^{+} \\
& +\int_{\tau_{u}^{+}=0}^{\tau_{k}^{+}} \int_{\varphi=0}^{\xi_{S}^{+}} T^{4}\left(\tau_{0}-\hat{u} \tan \varphi, \hat{y}_{k}-\hat{u}\right) K i_{2}\left(\frac{\tau_{u}^{+}}{\cos \varphi}\right) \tan \varphi d \varphi d \tau_{u}^{+}
\end{aligned}
$$

* Northern obstacle's surface radiosity temperature: 


$$
\begin{aligned}
\frac{\pi\left[T_{R \sigma_{N}}^{4}\left(\hat{x}_{k}\right)-\varepsilon_{\sigma_{N}} T_{\sigma_{N}}^{4}\right]}{2 \rho_{\sigma_{N}}} & \\
& =\int_{\varphi=0}^{\xi_{E}^{+}} T_{R N}^{4}\left(\tau_{k}^{+}+\tau_{0} \tan \varphi\right) K i_{3}\left(\frac{\tau_{0}}{\cos \varphi}\right) \cos \varphi d \varphi \\
& +\int_{\varphi=0}^{\xi_{E}^{-}} T_{R N}^{4}\left(\tau_{k}^{+}-\tau_{0} \tan \varphi\right) K i_{3}\left(\frac{\tau_{0}}{\cos \varphi}\right) \cos \varphi d \varphi \\
& +\int_{\varphi=0}^{\xi_{N}^{+}} T_{R E}^{4}\left(\tau_{0}^{\prime \prime}+\tau_{k}^{-} \tan \varphi\right) K i_{3}\left(\frac{\tau_{k}^{-}}{\cos \varphi}\right) \sin \varphi d \varphi \\
& +\int_{\varphi=0}^{\xi_{S}^{+}} T_{R O}^{4}\left(\tau^{\prime \prime}{ }_{0}+\tau_{k}^{+} \tan \varphi\right) K i_{3}\left(\frac{\tau_{k}^{+}}{\cos \varphi}\right) \sin \varphi d \varphi+\kappa \aleph_{\sigma_{N}}
\end{aligned}
$$

where the internal contribution $\aleph_{\sigma_{N}}$ writes in the general case of an non-isothermal medium:

$$
\begin{aligned}
\kappa \aleph_{\sigma_{N}}=\int_{\tau_{u}^{+}=0}^{\tau_{0}} & \left\{\int_{\varphi=0}^{\xi_{E}^{+}} T^{4}\left(\tau_{k}^{+}+\tau_{u}^{+} \tan \varphi, \tau_{u}^{+}+\tau_{0}{ }_{0}\right) K i_{2}\left(\frac{\tau_{u}^{+}}{\cos \varphi}\right) d \varphi\right. \\
& \left.+\int_{\varphi=0}^{\xi_{E}^{-}} T^{4}\left(\tau_{k}^{+}-\tau_{u}^{+} \tan \varphi, \tau_{u}^{+}+\tau_{0}{ }_{0}\right) K i_{2}\left(\frac{\tau_{u}^{+}}{\cos \varphi}\right) d \varphi\right\} d \tau_{u}^{+} \\
& +\int_{\tau_{u}^{+}=0}^{\tau_{k}^{-}} \int_{\varphi=0}^{\xi_{N}^{+}} T^{4}\left(\tau_{k}^{+}+\tau_{u}^{+}, \tau^{\prime \prime}{ }_{0}+\tau_{u}^{+} \tan \varphi\right) K i_{2}\left(\frac{\tau_{u}^{+}}{\cos \varphi}\right) \tan \varphi d \varphi d \tau_{u}^{+} \\
& +\int_{\tau_{u}^{+}=0}^{\tau_{k}^{+}} \int_{\varphi=0}^{\xi_{S}^{+}} T^{4}\left(\tau_{u}^{-}-\tau_{k}^{-}, \tau_{0}{ }_{0}+\tau_{u}^{+} \tan \varphi\right) K i_{2}\left(\frac{\tau_{u}^{+}}{\cos \varphi}\right) \tan \varphi d \varphi d \tau_{u}^{+}
\end{aligned}
$$

with the formal substitution $\aleph_{\sigma_{N}}\left(\hat{x}_{k}\right) \equiv \aleph_{\sigma_{E}}\left(\hat{y}_{k}\right)$, which for isothermal media reduces to:

$$
\frac{\kappa \aleph_{\sigma_{N}}}{T^{4}}=\frac{\pi}{2}-B i s_{3}\left(\tau_{0}, \xi_{E}^{+}\right)-B i s_{3}\left(\tau_{0}, \xi_{E}^{-}\right)-C i s_{3}\left(\tau_{k}^{-}, \xi_{N}^{+}\right)-C i s_{3}\left(\tau_{k}^{+}, \xi_{S}^{+}\right)
$$

or equivalently $\frac{\kappa \aleph}{T^{4}}\left(\hat{x}_{k} ; \sigma_{N}\right)=\frac{\kappa \aleph}{T^{4}}\left(\hat{y}_{k} ; \sigma_{E}\right)$

* Southern obstacle's surface radiosity temperature:

$$
\begin{aligned}
\frac{\pi\left[T_{R \sigma_{S}}^{4}\left(\hat{x}_{k}\right)-\varepsilon_{\sigma_{S}} T_{\sigma_{S}}^{4}\right]}{2 \rho_{\sigma_{S}}} & \\
& =\int_{\varphi=0}^{\xi_{E}^{+}} T_{R S}^{4}\left(\tau_{k}^{+}+\tau_{0} \tan \varphi\right) K i_{3}\left(\frac{\tau_{0}}{\cos \varphi}\right) \cos \varphi d \varphi \\
& +\int_{\varphi=0}^{\xi_{E}^{-}} T_{R S}^{4}\left(\tau_{k}^{+}-\tau_{0} \tan \varphi\right) K i_{3}\left(\frac{\tau_{0}}{\cos \varphi}\right) \cos \varphi d \varphi \\
& +\int_{\varphi=0}^{\xi_{N}^{+}} T_{R E}^{4}\left(\tau_{0}-\tau_{k}^{-} \tan \varphi\right) K i_{3}\left(\frac{\tau_{k}^{-}}{\cos \varphi}\right) \sin \varphi d \varphi \\
& +\int_{\varphi=0}^{\xi_{S}^{+}} T_{R O}^{4}\left(\tau_{0}-\tau_{k}^{+} \tan \varphi\right) K i_{3}\left(\frac{\tau_{k}^{+}}{\cos \varphi}\right) \sin \varphi d \varphi+\kappa \aleph_{S}
\end{aligned}
$$


where the exact internal contribution $\aleph_{S}$ is given by:

$$
\begin{aligned}
& \kappa \aleph_{S}=\int_{\tau_{u}^{+}=0}^{\tau_{0}}\left\{\int_{\varphi=0}^{\xi_{E}^{+}} T^{4}\left(\tau_{k}^{+}+\tau_{u}^{+} \tan \varphi, \tau_{0}-\tau_{u}^{+}\right) K i_{2}\left(\frac{\tau_{u}^{+}}{\cos \varphi}\right) d \varphi\right. \\
&\left.\quad+\int_{\varphi=0}^{\xi_{E}^{-}} T^{4}\left(\tau_{k}^{+}-\tau_{u}^{+} \tan \varphi, \tau_{0}-\tau_{u}^{+}\right) K i_{2}\left(\frac{\tau_{u}^{+}}{\cos \varphi}\right) d \varphi\right\} d \tau_{u}^{+} \\
&+\int_{\tau_{u}^{+}=0}^{\tau_{k}^{-}} \int_{\varphi=0}^{\xi_{N}^{+}} T^{4}\left(\tau_{k}^{+}+\tau_{u}^{+}, \tau_{0}-\tau_{u}^{+} \tan \varphi\right) K i_{2}\left(\frac{\tau_{u}^{+}}{\cos \varphi}\right) \tan \varphi d \varphi d \tau_{u}^{+} \\
&+\int_{\tau_{u}^{+}=0}^{\tau_{k}^{+}} \int_{\varphi=0}^{\xi_{S}^{+}} T^{4}\left(\tau_{k}^{+}-\tau_{u}^{+}, \tau_{0}-\tau_{u}^{+} \tan \varphi\right) K i_{2}\left(\frac{\tau_{u}^{+}}{\cos \varphi}\right) \tan \varphi d \varphi d \tau_{u}^{+}
\end{aligned}
$$

with the formal substitution $\aleph_{\sigma_{S}}\left(\hat{x}_{k}\right) \equiv \aleph_{\sigma_{O}}\left(\hat{y}_{k}\right)$, which keeps the expression (A5) of the northern contribution in the case of an isothermal medium.

\section{ANNEX 2: cavity's eastern radiosity temperature for points on the upper part of the surface}

- $\frac{h}{H} \leq \sqrt{5}-2$ or $\left(\frac{h}{H}>\sqrt{5}-2\right.$ and $\left.\frac{H}{2} \leq \hat{y}_{k} \leq \frac{H(H-h)}{H+h}\right)$, is equivalent to the case

$\frac{\mathrm{h}}{\mathrm{H}} \leq \sqrt{5}-2$ or $\left(\frac{\mathrm{h}}{\mathrm{H}}>\sqrt{5}-2\right.$ and $\left.\frac{2 \mathrm{hH}}{\mathrm{H}+\mathrm{h}} \leq \hat{\mathrm{y}}_{\mathrm{k}} \leq \frac{\mathrm{H}}{2}\right)$ with identical expressions of the radiosity temperature and isothermal medium contribution,

- $\left(\sqrt{5}-2 \leq \frac{h}{H}<\frac{1}{3}\right.$ and $\left.\frac{H(H-h)}{H+h} \leq \hat{y}_{k} \leq \frac{H+h}{2}\right)$ or $\left(\frac{h}{H} \geq \frac{1}{3}\right.$ and $\left.\frac{2 h H}{H+h} \leq \hat{y}_{k} \leq \frac{H+h}{2}\right)$, is equivalent to the case $\left(\sqrt{5}-2 \leq \frac{h}{H}<\frac{1}{3}\right.$ and $\left.\frac{H-h}{2} \leq \hat{y}_{k} \leq \frac{2 h H}{H+h}\right)$ or $\left(\frac{h}{H} \geq \frac{1}{3}\right.$ and $\left.\frac{H-h}{2} \leq \hat{y}_{k} \leq \frac{H(H-h)}{H+h}\right)$ with the appropriate substitutions:

$$
\begin{aligned}
\frac{\pi\left[T_{R E}^{4}\left(\hat{y}_{k}\right)-\varepsilon_{E} T_{E}^{4}\right]}{2 \rho_{E}} & \\
& =\int_{\varphi=0}^{\alpha_{S 1}^{+}} T_{R S}^{4}\left(\tau^{\prime}{ }_{0}-\tau_{k}^{+} \tan \varphi\right) K i_{3}\left(\frac{\tau_{k}^{+}}{\cos \varphi}\right) \sin \varphi d \varphi \\
& +\int_{\varphi=\alpha_{\sigma}^{+}}^{\alpha_{O}^{+}} T_{R O}^{4}\left(\tau_{k}^{+}+\tau_{0}^{\prime} \tan \varphi\right) K i_{3}\left(\frac{\tau_{0}^{\prime}}{\cos \varphi}\right) \cos \varphi d \varphi \\
& +\int_{\varphi=0}^{\alpha_{N}^{+}} T_{R N}^{4}\left(\tau_{0}^{\prime}-\tau_{k}^{-} \tan \varphi\right) K i_{3}\left(\frac{\tau_{k}^{-}}{\cos \varphi}\right) \sin \varphi d \varphi \\
& +\int_{\varphi=0}^{\alpha_{\sigma_{E}}^{+}} T_{R \sigma_{E}}^{4}\left(\tau_{k}^{+}+\tau_{0} \tan \varphi\right) K i_{3}\left(\frac{\tau_{0}}{\cos \varphi}\right) \cos \varphi d \varphi \\
& +\int_{\varphi=0}^{\alpha_{\sigma_{E}}} T_{R \sigma_{E}}^{4}\left(\tau_{k}^{+}-\tau_{0} \tan \varphi\right) K i_{3}\left(\frac{\tau_{0}}{\cos \varphi}\right) \cos \varphi d \varphi+\kappa \aleph_{E}
\end{aligned}
$$

In the latter expression the general internal contribution is described by: 


$$
\begin{aligned}
& \kappa \aleph_{E}=\int_{\varphi=0}^{\alpha_{S 1}^{+}} \int_{\tau_{u}^{+}=0}^{\tau_{k}^{+}} T^{4}\left[\tau_{0}^{\prime}-\left(\tau_{u}^{-}-\tau_{k}^{-}\right) \tan \varphi, \hat{u}\right] K i_{2}\left(\frac{\tau_{u}^{-}-\tau_{k}^{-}}{\cos \varphi}\right) d \tau_{u}^{+} \tan \varphi \tan \varphi d \varphi \\
&+\int_{\varphi=\alpha_{\sigma_{E}}^{+}}^{\alpha_{O}^{+}} \int_{\tau_{u}^{+}=0}^{\tau_{0}} T^{4}\left(\tau_{u}^{+}, \tau_{k}^{+}+\tau_{u}^{-} \tan \varphi\right) K i_{2}\left(\frac{\tau_{u}^{-}}{\cos \varphi}\right) d \tau_{u}^{+} d \varphi \\
&+\int_{\varphi=0}^{\alpha_{N}^{+}} \int_{\tau_{u}^{+}=\tau_{k}^{+}}^{\tau^{\prime} 0} T^{4}\left[\tau_{0}^{\prime}-\left(\tau_{u}^{+}-\tau_{k}^{+}\right) \tan \varphi, \tau_{u}^{+}\right] K i_{2}\left(\frac{\tau_{u}^{+}-\tau_{k}^{+}}{\cos \varphi}\right) d \tau_{u}^{+} d \varphi \\
&+\int_{\varphi=0}^{\alpha_{\sigma_{E}}^{+}} \int_{\tau_{u}^{+}=\tau_{0}^{\prime \prime}}^{\tau^{\prime} 0} T^{4}\left(\tau_{u}^{+}, \tau_{k}^{+}+\tau_{u}^{-} \tan \varphi\right) K i_{2}\left(\frac{\tau_{u}^{-}}{\cos \varphi}\right) d \tau_{u}^{+} d \varphi \\
&+\int_{\varphi=0}^{\alpha_{\sigma_{E}}^{-}} \int_{\tau_{u}^{+}=\tau_{0}^{\prime \prime}}^{\tau^{\prime}{ }_{0}} T^{4}\left(\tau_{u}^{+}, \tau_{k}^{+}-\tau_{u}^{-} \tan \varphi\right) K i_{2}\left(\frac{\tau_{u}^{-}}{\cos \varphi}\right) d \tau_{u}^{+} d \varphi
\end{aligned}
$$

whence the isothermal $\kappa \aleph_{E}$ function reduces to:

$$
\begin{gathered}
\frac{\kappa \aleph_{E}}{T^{4}}=\frac{\pi}{2}-B i s_{3}\left(\tau_{0}^{\prime}, \alpha_{O}^{+}\right)+B i s_{3}\left(\tau^{\prime}{ }_{0}, \alpha_{\sigma_{E}}^{-}\right)-B i s_{3}\left(\tau_{0}, \alpha_{\sigma_{E}}^{+}\right)-B i s_{3}\left(\tau_{0}, \alpha_{\sigma_{E}}^{-}\right)-C i s_{3}\left(\tau_{k}^{+}, \alpha_{S 1}^{+}\right) \\
-C i s_{3}\left(\tau_{k}^{-}, \alpha_{N}^{+}\right)
\end{gathered}
$$

- $\frac{h}{H} \geq \frac{1}{3}$ and $\frac{H}{2} \leq \hat{y}_{k} \leq \frac{2 h H}{H+h^{\prime}}$ is equivalent to the case $\frac{h}{H} \geq \frac{1}{3}$ and $\frac{H(H-h)}{H+h} \leq \hat{y}_{k} \leq \frac{H}{2}$, with the appropriate substitutions, which leads exactly to the same expressions for the radiosity temperature and isothermal medium contribution.

For the last configuration $\frac{H+h}{2} \leq \hat{y}_{k} \leq H$, where the angle $\alpha_{\sigma_{E}}^{+}$is now $\alpha_{\sigma_{E}}^{+}=\tan ^{-1}\left(\frac{2 \hat{y}_{k}-H-h}{H-h}\right)$, the latter variables change implies for the two considered cases, when introducing the two angles concerning the northern obstacle $\alpha_{\sigma_{N}}^{-}=\tan ^{-1}\left(\frac{H-h}{2 \hat{y}_{k}-H-h}\right)$ and $\alpha_{\sigma_{N}}^{+}=\tan ^{-1}\left(\frac{H+h}{2 \hat{y}_{k}-H-h}\right)$, and the latter angle $\alpha_{O 1}^{-}$for the western surface $\alpha_{O 1}^{-}=\frac{\pi}{2}-\alpha_{\sigma_{N}}^{+}=\tan ^{-1}\left(\frac{2 \hat{y}_{k}-H-h}{H+h}\right)$, with :

- $\frac{h}{H} \leq \sqrt{5}-2$ and $\frac{H+h}{2}<\hat{y}_{k} \leq \frac{H(H-h)}{H+h}$, 


$$
\begin{aligned}
\frac{\pi\left[T_{R E}^{4}\left(\hat{y}_{k}\right)-\varepsilon_{E} T_{E}^{4}\right]}{2 \rho_{E}} & \\
& =\int_{\varphi=0}^{\alpha_{N}^{+}} T_{R N}^{4}\left(\tau_{0}^{\prime}-\tau_{k}^{-} \tan \varphi\right) K i_{3}\left(\frac{\tau_{k}^{-}}{\cos \varphi}\right) \sin \varphi d \varphi \\
& +\int_{\varphi=0}^{\alpha_{O}^{+}} T_{R O}^{4}\left(\tau_{k}^{+}+\tau_{0}^{\prime} \tan \varphi\right) K i_{3}\left(\frac{\tau_{0}^{\prime}}{\cos \varphi}\right) \cos \varphi d \varphi \\
& +\int_{\varphi=0}^{\alpha_{\sigma_{E}}^{+}} T_{R O}^{4}\left(\tau_{k}^{+}-\tau_{0}^{\prime} \tan \varphi\right) K i_{3}\left(\frac{\tau_{0}^{\prime}}{\cos \varphi}\right) \cos \varphi d \varphi \\
& +\int_{\varphi=\alpha_{\sigma_{E}}}^{\alpha_{O}^{-}} T_{R O}^{4}\left(\tau_{k}^{+}-\tau_{0}^{\prime} \tan \varphi\right) K i_{3}\left(\frac{\tau^{\prime}}{\cos \varphi}\right) \cos \varphi d \varphi \\
& +\int_{\varphi=0}^{\alpha_{S}^{+}} T_{R S}^{4}\left(\tau_{0}^{\prime}-\tau_{k}^{+} \tan \varphi\right) K i_{3}\left(\frac{\tau_{k}^{+}}{\cos \varphi}\right) \sin \varphi d \varphi \\
& +\int_{\varphi=\alpha_{\sigma_{E}}^{+}}^{\alpha_{\sigma_{E}}} T_{R \sigma_{E}}^{4}\left(\tau_{k}^{+}-\tau_{0} \tan \varphi\right) K i_{3}\left(\frac{\tau_{0}}{\cos \varphi}\right) \cos \varphi d \varphi \\
& +\int_{\varphi=\alpha_{\sigma_{N}}^{-}}^{\alpha_{\sigma_{N}}^{+}} T_{R \sigma_{N}}^{4}\left[\tau_{0}^{\prime}-\left(\tau_{0}-\tau_{k}^{-}\right) \tan \varphi\right] K i_{3}\left(\frac{\tau_{0}-\tau_{k}^{-}}{\cos \varphi}\right) \sin \varphi d \varphi+\kappa \aleph_{E}
\end{aligned}
$$

where the internal contribution $\kappa \aleph_{E}$ is similarly given by:

$$
\begin{aligned}
& \kappa \aleph_{E}=\int_{\varphi=0}^{\alpha_{N}^{+}} \int_{\tau_{u}^{+}=\tau_{k}^{+}}^{\tau_{0}^{\prime}} T^{4}\left[\tau_{0}^{\prime}-\left(\tau_{u}^{+}-\tau_{k}^{+}\right) \tan \varphi, \tau_{u}^{+}\right] K i_{2}\left(\frac{\tau_{u}^{+}-\tau_{k}^{+}}{\cos \varphi}\right) d \tau_{u}^{+} \tan \varphi d \varphi \\
&+\int_{\varphi=0}^{\alpha_{O}^{+}} \int_{\tau_{u}^{+}=0}^{\tau_{0}^{\prime}} T^{4}\left(\tau_{u}^{+}, \tau_{k}^{+}+\tau_{u}^{-} \tan \varphi\right) K i_{2}\left(\frac{\tau_{u}^{-}}{\cos \varphi}\right) d \tau_{u}^{+} d \varphi \\
&+\int_{\varphi=0}^{\alpha_{\sigma_{E}}^{+}} \int_{\tau_{u}^{+}=0}^{\tau_{0}^{\prime}} T^{4}\left(\tau_{u}^{+}, \tau_{k}^{+}-\tau_{u}^{-} \tan \varphi\right) K i_{2}\left(\frac{\tau_{u}^{-}}{\cos \varphi}\right) d \tau_{u}^{+} d \varphi \\
&+\int_{\varphi=\alpha_{\sigma_{E}}^{-}}^{\alpha_{O}^{-}} \int_{\tau_{u}^{+}=0}^{\tau_{0}^{\prime}} T^{4}\left(\tau_{u}^{+}, \tau_{k}^{+}-\tau_{u}^{-} \tan \varphi\right) K i_{2}\left(\frac{\tau_{u}^{-}}{\cos \varphi}\right) d \tau_{u}^{+} d \varphi \\
&+\int_{\varphi=0}^{\alpha_{S}^{+}} \int_{\tau_{u}^{+}=0}^{\tau_{k}^{+}} T^{4}\left[\tau^{\prime}{ }_{0}-\left(\tau_{u}^{-}-\tau_{k}^{-}\right) \tan \varphi, \tau_{u}^{+}\right] K i_{2}\left(\frac{\tau_{u}^{-}-\tau_{k}^{-}}{\cos \varphi}\right) d \tau_{u}^{+} \tan \varphi d \varphi \\
&+\int_{\varphi=\alpha_{\sigma_{E}}^{+}}^{\alpha_{\sigma_{E}}} \int_{\tau_{u}^{+}=\tau_{0}^{\prime \prime}}^{\tau_{\prime}{ }_{0}} T^{4}\left(\tau_{u}^{+}, \tau_{k}^{+}-\tau_{u}^{-} \tan \varphi\right) K i_{2}\left(\frac{\tau_{u}^{-}}{\cos \varphi}\right) d \tau_{u}^{+} d \varphi \\
&+\int_{\varphi=\alpha_{\sigma_{N}}^{-}}^{\alpha_{\sigma_{N}}} \int_{\tau_{u}^{+}=\tau_{0}}^{\tau_{k}^{+}} T^{4}\left[\tau_{0}^{\prime}-\left(\tau_{u}^{-}-\tau_{k}^{-}\right) \tan \varphi, \tau_{u}^{+}\right] K i_{2}\left(\frac{\tau_{u}^{-}-\tau_{k}^{-}}{\cos \varphi}\right) d \tau_{u}^{+} \tan \varphi d \varphi
\end{aligned}
$$

which for isothermal media reduces to:

$$
\begin{aligned}
\frac{\kappa \aleph_{E}}{T^{4}}=\frac{\pi}{2}-B i S_{3}\left(\tau^{\prime}{ }_{0}, \alpha_{O}^{-}\right)+B i s_{3}\left(\tau^{\prime}{ }_{0}, \alpha_{\sigma_{E}}^{-}\right)-B i s_{3}\left(\tau^{\prime}{ }_{0}, \alpha_{O}^{+}\right)-B i s_{3}\left(\tau^{\prime}{ }_{0}, \alpha_{\sigma_{E}}^{+}\right)-B i s_{3}\left(\tau_{0}, \alpha_{\sigma_{E}}^{-}\right) \\
+B i s_{3}\left(\tau_{0}, \alpha_{\sigma_{E}}^{+}\right)-C_{i} i_{3}\left(\tau_{k}^{-}, \alpha_{N}^{+}\right)-\operatorname{Cis}_{3}\left(\tau_{k}^{+}, \alpha_{S}^{+}\right)-C i s_{3}\left(\tau_{0}-\tau_{k}^{-}, \alpha_{\sigma_{N}}^{+}\right) \\
+C i s_{3}\left(\tau_{0}-\tau_{k}^{-}, \alpha_{\sigma_{N}}^{-}\right)
\end{aligned}
$$


- $\frac{h}{H} \leq \sqrt{5}-2$ and $\frac{H(H-h)}{H+h} \leq \hat{y}_{k} \leq H$ or $\frac{h}{H}>\sqrt{5}-2$ and $\frac{H+h}{2} \leq \hat{y}_{k} \leq H$,

$$
\begin{aligned}
\frac{\pi\left[T_{R E}^{4}\left(\hat{y}_{k}\right)-\varepsilon_{E} T_{E}^{4}\right]}{2 \rho_{E}} & \\
& =\int_{\varphi=0}^{\alpha_{N}^{+}} T_{R N}^{4}\left(\tau^{\prime}{ }_{0}-\tau_{k}^{-} \tan \varphi\right) K i_{3}\left(\frac{\tau_{k}^{-}}{\cos \varphi}\right) \sin \varphi d \varphi \\
& +\int_{\varphi=0}^{\alpha_{O}^{+}} T_{R O}^{4}\left(\tau_{k}^{+}+\tau_{0}^{\prime} \tan \varphi\right) K i_{3}\left(\frac{\tau_{0}^{\prime}}{\cos \varphi}\right) \cos \varphi d \varphi \\
& +\int_{\varphi=0}^{\alpha_{O 1}^{-}} T_{R O}^{4}\left(\tau_{k}^{+}-\tau_{0}^{\prime} \tan \varphi\right) K i_{3}\left(\frac{\tau_{0}^{\prime}}{\cos \varphi}\right) \cos \varphi d \varphi \\
& +\int_{\varphi=0}^{\alpha_{S 1}^{+}} T_{R S}^{4}\left(\tau^{\prime}{ }_{0}-\tau_{k}^{+} \tan \varphi\right) K i_{3}\left(\frac{\tau_{k}^{+}}{\cos \varphi}\right) \sin \varphi d \varphi \\
& +\int_{\varphi=\alpha_{\sigma_{E}}^{+}}^{\alpha_{\sigma_{E}}} T_{R \sigma_{E}}^{4}\left(\tau_{k}^{+}-\tau_{0} \tan \varphi\right) K i_{3}\left(\frac{\tau_{0}}{\cos \varphi}\right) \cos \varphi d \varphi \\
& +\int_{\varphi=\alpha_{\sigma_{N}}^{-}}^{\alpha_{\sigma_{N}}^{+}} T_{R \sigma_{N}}^{4}\left[\tau^{\prime}{ }_{0}-\left(\tau_{0}-\tau_{k}^{-}\right) \tan \varphi\right] K i_{3}\left(\frac{\tau_{0}-\tau_{k}^{-}}{\cos \varphi}\right) \sin \varphi d \varphi+\kappa \aleph_{E}
\end{aligned}
$$

where the global internal contribution is expressed by:

$$
\begin{aligned}
& \kappa \aleph_{E}=\int_{\varphi=0}^{\alpha_{N}^{+}} \int_{\tau_{u}^{+}=\tau_{k}^{+}}^{\tau \prime} T^{4}\left[\tau_{0}^{\prime}-\left(\tau_{u}^{+}-\tau_{k}^{+}\right) \tan \varphi, \tau_{u}^{+}\right] K i_{2}\left(\frac{\tau_{u}^{+}-\tau_{k}^{+}}{\cos \varphi}\right) d \tau_{u}^{+} \tan \varphi d \varphi \\
&+\int_{\varphi=0}^{\alpha_{O}^{+}} \int_{\tau_{u}^{+}=0}^{\tau_{0}^{\prime}} T^{4}\left(\tau_{u}^{+}, \tau_{k}^{+}+\tau_{u}^{-} \tan \varphi\right) K i_{2}\left(\frac{\tau_{u}^{-}}{\cos \varphi}\right) d \tau_{u}^{+} d \varphi \\
&+\int_{\varphi=0}^{\alpha_{O 1}^{-}} \int_{\tau_{u}^{+}=0}^{\tau_{0}^{\prime}} T^{4}\left(\tau_{u}^{+}, \tau_{k}^{+}-\tau_{u}^{-} \tan \varphi\right) K i_{2}\left(\frac{\tau_{u}^{-}}{\cos \varphi}\right) d \tau_{u}^{+} d \varphi \\
&+\int_{\varphi=0}^{\alpha_{S 1}^{+}} \int_{\tau_{u}^{+}=0}^{\tau_{k}^{+}} T^{4}\left[\tau_{0}^{\prime}-\left(\tau_{u}^{-}-\tau_{k}^{-}\right) \tan \varphi, \tau_{u}^{+}\right] K i_{2}\left(\frac{\tau_{u}^{-}-\tau_{k}^{-}}{\cos \varphi}\right) d \tau_{u}^{+} \tan \varphi d \varphi \\
&+\int_{\varphi=\alpha_{\sigma_{E}}^{+}}^{\alpha_{\sigma_{E}}} \int_{\tau_{u}^{+}=\tau_{0}{ }_{0}}^{\tau_{\prime}{ }_{0}} T^{4}\left(\tau_{u}^{+}, \tau_{k}^{+}-\tau_{u}^{-} \tan \varphi\right) K i_{2}\left(\frac{\tau_{u}^{-}}{\cos \varphi}\right) d \tau_{u}^{+} d \varphi \\
&+\int_{\varphi=\alpha_{\sigma_{N}}}^{\alpha_{\sigma_{N}}} \int_{\tau_{u}^{+}=\tau_{0}}^{\tau_{k}^{+}} T^{4}\left[\tau_{0}^{\prime}-\left(\tau_{u}^{-}-\tau_{k}^{-}\right) \tan \varphi, \tau_{u}^{+}\right] K i_{2}\left(\frac{\tau_{u}^{-}-\tau_{k}^{-}}{\cos \varphi}\right) d \tau_{u}^{+} \tan \varphi d \varphi
\end{aligned}
$$

and leading to for internal media:

$$
\begin{gathered}
\frac{\kappa \aleph_{E}}{T^{4}}=\frac{\pi}{2}-B i s_{3}\left(\tau^{\prime}{ }_{0}, \alpha_{O}^{+}\right)-B i s_{3}\left(\tau_{0}^{\prime}, \alpha_{O 1}^{-}\right)-B i s_{3}\left(\tau_{0}, \alpha_{\sigma_{E}}^{-}\right)+B i s_{3}\left(\tau_{0}, \alpha_{\sigma_{E}}^{+}\right)-C i s_{3}\left(\tau_{k}^{-}, \alpha_{N}^{+}\right) \\
-C i s_{3}\left(\tau_{k}^{+}, \alpha_{S 1}^{+}\right)-C i s_{3}\left(\tau_{0}-\tau_{k}^{-}, \alpha_{\sigma_{N}}^{+}\right)+C i s_{3}\left(\tau_{0}-\tau_{k}^{-}, \alpha_{\sigma_{N}}^{-}\right)
\end{gathered}
$$


If $\frac{h}{H} \leq \sqrt{5}-2$ and $\frac{2 h H}{H+h}<\hat{y}_{k} \leq \frac{H-h}{2}$, the northern contribution is obtained from:

$$
\begin{aligned}
\int_{\varphi=0}^{\alpha_{N}^{+}} T_{R N}^{4}\left(\tau_{0}^{\prime}-\right. & \left.\tau_{k}^{-} \tan \varphi\right) K i_{3}\left(\frac{\tau_{k}^{-}}{\cos \varphi}\right) \sin \varphi d \varphi \\
& =\sum_{m=2}^{M-1} T_{R N, m}^{4} \int_{\varphi=\varphi_{m}^{-}}^{\varphi_{m}^{+}} K i_{3}\left(\frac{\tau_{k}^{-}}{\cos \varphi}\right) \sin \varphi d \varphi \\
& +\sum_{m=M+1}^{M+P-2} T_{R N, m}^{4} \int_{\varphi=\varphi_{m}^{-}}^{\varphi_{m}^{+}} K i_{3}\left(\frac{\tau_{k}^{-}}{\cos \varphi}\right) \sin \varphi d \varphi \\
& +\sum_{m=M+P}^{M+2 P-3} T_{R N, m}^{4} \int_{\varphi=\varphi_{m}^{-}}^{\varphi_{m}^{+}} K i_{3}\left(\frac{\tau_{k}^{-}}{\cos \varphi}\right) \sin \varphi d \varphi \\
& +\sum_{m=M+2 P-1}^{2(M+P-2)} T_{R N, m}^{4} \int_{\varphi=\varphi_{m}^{-}}^{\varphi_{m}^{+}} K i_{3}\left(\frac{\tau_{k}^{-}}{\cos \varphi}\right) \sin \varphi d \varphi
\end{aligned}
$$

with $\varphi_{m}^{-}=\tan ^{-1}\left(\frac{H-\hat{x}_{m}-\frac{\Delta \widehat{x}}{2}}{H-\hat{y}_{k}}\right)$ and $\varphi_{m}^{+}=\tan ^{-1}\left(\frac{H-\hat{x}_{m}+\frac{\Delta \widehat{x}}{2}}{H-\hat{y}_{k}}\right)$ for any point $M_{k}$ on $\Sigma_{E} \operatorname{such} \frac{2 h H}{H+h}<\hat{y}_{k} \leq \frac{H-h}{2}$, which can be summed up under the formal expression:

$$
\int_{\varphi=0}^{\alpha_{N}^{+}} T_{R N}^{4}\left(\tau^{\prime}{ }_{0}-\tau_{k}^{-} \tan \varphi\right) K i_{3}\left(\frac{\tau_{k}^{-}}{\cos \varphi}\right) \sin \varphi d \varphi=\sum_{m=2}^{2(M+P-2)} C_{k, m}^{N, E} T_{R N, m}^{4}
$$

where $C_{k, m}^{N, E}=C i s_{3}\left(\tau_{k}^{-}, \varphi_{m}^{+}\right)-C i s_{3}\left(\tau_{k}^{-}, \varphi_{m}^{-}\right)$and $C_{k, M}^{N, E}=C_{k, M+P-1}^{N, E}=C_{k, M+2 P-2}^{N, E}=0$

Similarly for the southern contribution, one obtains:

$$
\int_{\varphi=0}^{\alpha_{S}^{+}} T_{R S}^{4}\left(\tau_{0}^{\prime}-\tau_{k}^{+} \tan \varphi\right) K i_{3}\left(\frac{\tau_{k}^{+}}{\cos \varphi}\right) \sin \varphi d \varphi=\sum_{m=2}^{2(M+P-2)} C_{k, m}^{S, E} T_{R S, m}^{4}
$$

where the coefficients $C_{k, m}^{S, E}$ are given by $C_{k, M}^{S, E}=C_{k, M+P-1}^{S, E}=C_{k, M+2 P-2}^{S, E}=0$ and $C_{k, m}^{S, E}=\operatorname{Cis}_{3}\left(\tau_{k}^{+}, \varphi_{m}^{+}\right)-\operatorname{Cis}_{3}\left(\tau_{k}^{+}, \varphi_{m}^{-}\right)$for $m \neq\{M, M+P-1, M+2 P-2\}$

The whole obstacle's eastern surface being seen in this configuration, its contribution to $\Sigma_{E}$ writes as for the two previous surfaces:

$$
\begin{aligned}
\int_{\varphi=\alpha_{\sigma_{E}}^{-}}^{\alpha_{\sigma_{E}}^{+}} T_{R \sigma_{E}}^{4}\left(\tau_{k}^{+}+\tau_{0} \tan \varphi\right) K i_{3}\left(\frac{\tau_{k}^{-}}{\cos \varphi}\right) \cos \varphi d \varphi \\
=\sum_{m=M+1}^{M+P-2} T_{R \sigma_{E}, m}^{4} \int_{\varphi=\varphi_{m}^{-}}^{\varphi_{m}^{+}} K i_{3}\left(\frac{\tau_{0}}{\cos \varphi}\right) \cos \varphi d \varphi \\
+\sum_{m=M+P}^{M+2 P-3} T_{R \sigma_{E}, m}^{4} \int_{\varphi=\varphi_{m}^{-}}^{\varphi_{m}^{+}} K i_{3}\left(\frac{\tau_{0}}{\cos \varphi}\right) \cos \varphi d \varphi
\end{aligned}
$$


with $\varphi_{m}^{-}=\tan ^{-1}\left[\frac{2\left(\hat{y}_{m}-\hat{y}_{k}\right)-\Delta \hat{y}}{H-h}\right]$ and $\varphi_{m}^{+}=\tan ^{-1}\left[\frac{2\left(\hat{y}_{m}-\hat{y}_{k}\right)+\Delta \hat{y}}{H-h}\right]$ for any point $M_{k}$, whence:

$$
\int_{\varphi=\alpha_{\sigma_{E}}^{-}}^{\alpha_{\sigma_{E}}^{+}} T_{R \sigma_{E}}^{4}\left(\tau_{k}^{+}+\tau_{0} \tan \varphi\right) K i_{3}\left(\frac{\tau_{k}^{-}}{\cos \varphi}\right) \cos \varphi d \varphi=\sum_{m=M+1}^{M+2 P-3} C_{k, m}^{\sigma_{E}, E} T_{R \sigma_{E}, m}^{4}
$$

where $C_{k, M+P-1}^{\sigma_{E}, E}=0$ and $C_{k, m}^{\sigma_{E}, E}=B i s_{3}\left(\tau_{0}, \varphi_{m}^{+}\right)-B i s_{3}\left(\tau_{0}, \varphi_{m}^{-}\right)$

Similarly one obtains for the southern obstacle's surface contribution:

$$
\int_{\varphi=\alpha_{\sigma_{S}}^{-}}^{\alpha_{\sigma_{S}}^{+}} T_{R \sigma_{S}}^{4}\left[\tau^{\prime}{ }_{0}-\left(\tau_{0}-\tau_{k}^{+}\right) \tan \varphi\right] K i_{3}\left(\frac{\tau_{0}-\tau_{k}^{+}}{\cos \varphi}\right) \sin \varphi d \varphi=\sum_{m=M+1}^{M+2 P-3} C_{k, m}^{\sigma_{S}, E} T_{R \sigma_{S}, m}^{4}
$$

with $C_{k, M+P-1}^{\sigma_{S}, E}=0$ and

$C_{k, m}^{\sigma_{S}, E}=\operatorname{Cis}_{3}\left\{\tau_{0}-\tau_{k}^{+}, \tan ^{-1}\left[\frac{2\left(H-\hat{x}_{m}\right)+\Delta \hat{x}}{H-h-2 \hat{y}_{k}}\right]\right\}-\operatorname{Cis}_{3}\left\{\tau_{0}-\tau_{k}^{+}, \tan ^{-1}\left[\frac{2\left(H-\hat{x}_{m}\right)-\Delta \hat{x}}{H-h-2 \hat{y}_{k}}\right]\right\}$

The contribution of the lower part of $\Sigma_{O}$ writes for a non-punctual cell $k \neq M$ :

$$
\int_{\varphi=0}^{\alpha_{O}^{-}} T_{R O}^{4}\left(\tau_{k}^{+}-\tau_{0}^{\prime} \tan \varphi\right) K i_{3}\left(\frac{\tau^{\prime}{ }_{0}}{\cos \varphi}\right) \cos \varphi d \varphi=\sum_{m=2}^{k} C_{k, m}^{O, E} T_{R O, m}^{4}
$$

where the coefficients $C_{k, m}^{O, E}$ are such that $C_{k, k}^{O, E}=B i s_{3}\left[\tau^{\prime}{ }_{0}, \tan ^{-1}\left(\frac{\Delta \hat{y}}{2 H}\right)\right]$ and

$C_{k, m}^{O, E}=B i s_{3}\left(\tau_{0}^{\prime}, \varphi_{m}^{+}\right)-B i s_{3}\left(\tau_{0}^{\prime}, \varphi_{m}^{-}\right)$for $2 \leq m \leq k-1$

If $k=M$, i.e. $\hat{y}_{k}=\frac{H-h}{2}, C_{k, k}^{O, E}=0$ and $C_{k, k-1}^{O, E}=B i s_{3}\left[\tau^{\prime}{ }_{0}, \tan ^{-1}\left(\frac{\Delta \hat{y}}{H}\right)\right]$

For the upper part below the obstacle, the contribution writes, for $k \neq M$ :

$$
\int_{\varphi=0}^{\alpha_{O 1}^{+}} T_{R O}^{4}\left(\tau_{k}^{+}+\tau_{0}^{\prime} \tan \varphi\right) K i_{3}\left(\frac{\tau_{0}^{\prime}}{\cos \varphi}\right) \cos \varphi d \varphi=C_{k, k}^{O, E+} T_{R O, k}^{4}+\sum_{m=k+1}^{p-1} C_{k, m}^{O, E} T_{R O, m}^{4}+C_{k, p}^{O, E} T_{R O, p}^{4}
$$

where $p$ is the index such that the point of ordinate $\tilde{y}=\hat{y}_{k}+\frac{H\left(H-h-2 \hat{y}_{k}\right)}{H+h}=\frac{\left(H-\hat{y}_{k}\right)(H-h)}{H+h}$ on the cavity's western surface belongs to the cell of number $p$, i. e. $\hat{y}_{p}-\frac{\Delta \hat{y}}{2} \leq \tilde{y}<\hat{y}_{p}+\frac{\Delta \hat{y}}{2}$, which can be summed up as

$$
\begin{aligned}
& 3+\llbracket \frac{\tilde{y}}{\Delta \hat{y}} \rrbracket \quad \tilde{y}<\frac{H}{2} \\
& p=4+\llbracket \frac{\tilde{y}}{\Delta \hat{y}} \rrbracket \quad \text { if } \quad \frac{H}{2}<\tilde{y}<\frac{H+h}{2} \text { with } \llbracket x \rrbracket \text { the integer part of a real } x \text {, or } p=\begin{array}{c}
M+P-1 \\
M+2 P-2
\end{array} \quad \text { if } \begin{array}{c}
\tilde{y}=\frac{H}{2} \\
\tilde{y}=\frac{H+h}{2}
\end{array} \\
& 5+\llbracket \frac{\tilde{y}}{\Delta \hat{y}} \rrbracket \quad \tilde{y}>\frac{H+h}{2}
\end{aligned}
$$

In these conditions, the coefficients $C_{k, m}^{O, E}$ write $C_{k, k}^{O, E+}=B i s_{3}\left[\tau^{\prime}{ }_{0}, \tan ^{-1}\left(\frac{\Delta \hat{y}}{2 H}\right)\right]$,

$C_{k, p}^{O, E}=B i s_{3}\left[\tau^{\prime}{ }_{0}, \tan ^{-1}\left(\frac{H-h-2 \hat{y}_{k}}{H+h}\right)\right]-B i s_{3}\left[\tau_{0}^{\prime}, \tan ^{-1}\left(\frac{\hat{y}_{p}-\hat{y}_{k}-\frac{\Delta \widehat{y}}{2}}{H}\right)\right]$ and

$C_{k, m}^{O, E}=B i s_{3}\left[\tau^{\prime}{ }_{0}, \tan ^{-1}\left(\frac{\hat{y}_{m}-\hat{y}_{k}+\frac{\Delta \hat{y}}{2}}{H}\right)\right]-B i s_{3}\left[\tau^{\prime}{ }_{0}, \tan ^{-1}\left(\frac{\hat{y}_{m}-\hat{y}_{k}-\frac{\Delta \hat{y}}{2}}{H}\right)\right]$ for $k+1 \leq m \leq p-1$ 
The treatment of the upper part above the obstacle is performed similarly by determining first the index $q$ such that $\tilde{y}=\hat{y}_{k}+\frac{H\left(H+h-2 \hat{y}_{k}\right)}{H+h}=H-\frac{H-h}{H+h} \hat{y}_{k}$ on the cavity's western surface belongs to the cell of number $q$, given by $q=5+\llbracket \frac{\tilde{y}}{\Delta \hat{y}} \rrbracket$ since $\tilde{y}$ is always greater than $\frac{H+h}{2}$, from which the last contribution writes, for $k \leq M$ :

$$
\int_{\varphi=\alpha_{\sigma_{E}}^{+}}^{\alpha_{O}^{+}} T_{R O}^{4}\left(\tau_{k}^{+}+\tau_{0}^{\prime} \tan \varphi\right) K i_{3}\left(\frac{\tau_{0}^{\prime}}{\cos \varphi}\right) \cos \varphi d \varphi=C_{k, q}^{O, E} T_{R O, q}^{4}+\sum_{m=q+1}^{2(M+P-2)} C_{k, m}^{O, E} T_{R O, m}^{4}
$$

where the different coefficients $C_{k, m}^{O, E}$ are given by

$$
\begin{aligned}
& C_{k, q}^{O, E}=B i s_{3}\left[\tau_{0}^{\prime}{ }_{0} \tan ^{-1}\left(\frac{\hat{y}_{q}-\hat{y}_{k}+\frac{\Delta \hat{y}}{2}}{H}\right)\right]-B i s_{3}\left[\tau_{0}^{\prime}{ }_{0}, \tan ^{-1}\left(\frac{H+h-2 \hat{y}_{k}}{H-h}\right)\right], \\
& C_{k, m}^{O, E}=B i s_{3}\left[\tau_{0}^{\prime}{ }_{0}, \tan ^{-1}\left(\frac{\hat{y}_{m}-\hat{y}_{k}+\frac{\Delta \hat{y}}{2}}{H}\right)\right]-B i s_{3}\left[\tau^{\prime}{ }_{0}, \tan ^{-1}\left(\frac{\hat{y}_{m}-\hat{y}_{k}-\frac{\Delta \hat{y}}{2}}{H}\right)\right] \text { for } q+1 \leq m \leq 2(M+P)-5, \\
& \text { and } C_{k, 2(M+P-2)}^{O, E}=B i s_{3}\left[\tau_{0}^{\prime}{ }_{0}, \tan ^{-1}\left(\frac{H-\hat{y}_{k}}{H}\right)\right]-B i s_{3}\left[\tau^{\prime}{ }_{0}, \tan ^{-1}\left(\frac{\hat{y}_{2(M+P-2)}-\hat{y}_{k}-\frac{\Delta \hat{y}}{2}}{H}\right)\right]
\end{aligned}
$$

\section{REFERENCES}

[1] A. Bahlaoui, A. Raji, M. Hasnaoui, C. Ouardi, M. Naïmi and T. Makayssi: Height Partition Effect on Combined Mixed Convection and Surface Radiation in a Vented Rectangular Cavity, Journal of Applied Fluid Mechanics, Vol. 4, No.1, pp. 89-96 (2011)

[2] A. Baïri, N. Laraqi and J.M Garcia de Maria: Importance of radiative heat exchange in 2D diode cavities applied to solar collectors and building, Int. Journal of Sustainable Energy, Vol. 24 No 1, pp. 33$44(2005)$

[3] Z. Tan and J.R. Howell: Combined radiation and natural convection in a two dimensional participating square medium, Int. J. Heat Mass. Transfer, Vol. 34 No 3, pp. 785-793 (1991)

[4] F.R. Steward and P. Cannon: The calculation of radiative heat flux in a cylindrical furnace using the Monte-Carlo method, IJHMT vol. 14, pp. 245-261 (1971)

[5] V. Le Dez, R. Vaillon, D. Lemonnier and M. Lallemand: Conductive-radiative coupling in an absorbing-emitting axisymmetric medium, Journal of Quant. Spectrosc. Radiat. Transfer, Vol. 65, pp. 787-803 (2000)

[6] G. Colomer, M. Costa, R. Consul, A. Oliva: Three-dimensional numerical simulation of convection and radiation in a differentially heated cavity using the discrete ordinates method, Int. J. Heat Mass Transfer, Vol. 47, pp. 257-269 (2004)

[7] C.Y. Han, S.W. Baek: The effects of radiation on natural convection in a rectangular enclosure divided by two partitions, Numer. Heat Transfer A, Vol. 37 No 3, pp. 249-270 (2000)

[8] S.C. Mishra, H.K. Roy and N. Misra: Discrete ordinate method with a new and a simple quadrature scheme, JQSRT vol. 101, n², pp. 249-262 (2006)

[9] B. W. Li, Y. S. Sun and D. W. Zhang: Chebyshev collocation spectral methods for coupled radiation and conduction in a concentric spherical participating medium, Journal of Heat Transfer, Vol. 131 No 6, pp. 062701-1/9 (2009)

[10] D. Le Hardy, Y. Favennec, B. Rousseau et F. Hecht: Specular reflection treatment for the 3D radiative transfer equation solved with the discrete ordinates method, Journal of Computational Physics, vol. 334 no. 1, pp. 541-572 (2017)

[11] C. Prax, P. Salagnac and H. Sadat: Diffuse approximation and control-volume-based finite-element methods: a comparative study, Numerical Heat Transfer Part B Fundamentals, vol. 34 n³, pp. 303-321 (1998) 
[12] H. Sadat, C. Wang and V. Le Dez: Meshless method for solving coupled radiative and conductive heat transfer in complex multi-dimensional geometries, Applied Mathematics and Computation, Vol. 218, pp. 10211-10225 (2012)

[13] V. Le Dez and H. Sadat: Radiative heat transfer in a parallelogram shaped cavity, Int. Com. In Heat and Mass Transfer, Vol. 68, pp. 137-149 (2015)

[14] V. Le Dez, H. Sadat and C. Prax: Radiative heat transfer in an absorbing-emitting semi-transparent medium enclosed in a two-dimensional parallelogram cavity with diffuse reflecting boundaries, Int. Journal of Thermal Sciences, Vol. 134, pp. 392-409 (2018)

[15] M. Lazard: Heat transfer in a semi-transparent parallelogram shaped medium, Int. Journal of Heat and Technology, Vol. 34 No 2, pp. 420-424 (2016)

[16] Z. Altaç and M. Tekkalmaz: Benchmark solutions of radiative transfer equation for threedimensional rectangular homogeneous media, JQSRT vol. 109, nº4, pp. 587-607 (2008)

[17] A.L. Crosbie and R.G. Schrenker: Radiative transfer in a two-dimensional rectangular medium exposed to diffuse radiation, JQSRT vol. 31, n4, pp. 339-372 (1984)

[18] V. Le Dez, H. Sadat and C. Prax, Radiative transfer in a semi-transparent medium enclosed in a spherical annulus with specularly reflecting surfaces, Int. Journal of Thermal Science, vol. 138 pp. 47-60, 2019

[19] R. Ul Haq, F. A. Soomro, H. F. Öztop and T. Mekkaoui: Thermal management of water-based carbon nanotubes enclosed in a partially heated triangular cavity with heated cylindrical obstacle, Int. Journal Heat Mass Transfer, Vol. 131, pp. 724-736 (2019)

[20] A. Mezrhab, H. Bouali, H. Amaoui and M. Bouzidi: Computation of combined natural-convection and radiation heat transfer in a cavity having a square body at its center, Applied Energy, Vol. 83, pp. 1004-1023 (2006)

[21] P. J. Coelho, J. M. Gonçalves and M. G. Carvalho: Modelling of radiative heat transfer in enclosures with obstacles, Int. Journal Heat Mass Transfer, Vol. 41, pp. 745-756 (1997)

[22] M. Sakami, A. El Kasmi and A. Charette: Analysis of radiative heat transfer in complex twodimensional enclosures with obstacles using the modified discrete ordinates method, Journal of Heat Transfer, Vol. 123 No 5, pp. 892-900 (2001)

[23] C. H. Wang and H. Y. Liu: Discontinuous Galerkin finite element method for radiative heat transfer in two-dimensional media with inner obstacles, numerical heat Transfer part A, Vol. 73 No 11, pp. 806$822(2018)$

[24] J. S. Djeumegni, M. Lazard, V. Le Dez and H. T. Tagne Kandem: Radiative heat transfer in a 2D semi-transparent gray medium with a centered inner square cavity, Int. Journal of Heat Mass Transfer, Vol. 149, 19p (2020)

[25] J. S. Djeumegni, M. Lazard, V. Le Dez and H. T. Tagne Kandem,Conductive-radiative heat transfer in a $2 \mathrm{D}$ semi-transparent medium with a square centered obstacle, International Journal of Thermal Sciences, Vol.159, 17p (2021)

[26] Z. Altac: Integrals involving Bickley and Bessel functions in radiative transfer and generalized exponential integral function, J Heat Transfer vol. 118, n³, pp. 789-791 (1996)

[27] Z. Altaç and M. Tekkalmaz: Solution of the radiative integral transfer equations in rectangular participating and isotropically scattering inhomogeneous medium, Int. Journal Heat Mass Transfer, Vol.

47, pp 101-109 (2004)

[28] D. E. Amos (Sandra National Laboratories): Algorithm 609: a portable Fortran subroutine for the Bickley functions $K i_{n}(x)$, ACM Transactions on Mathematical Software, Vol. 9 No 4 (1983) 
